Rochester Institute of Technology

RIT Scholar Works

Theses

8-1-2007

\title{
An evolutionary algorithm approach to simultaneous multi- mission radar waveform design
}

Jason Enslin

Follow this and additional works at: https://scholarworks.rit.edu/theses

\section{Recommended Citation}

Enslin, Jason, "An evolutionary algorithm approach to simultaneous multi-mission radar waveform design" (2007). Thesis. Rochester Institute of Technology. Accessed from 
An Evolutionary Algorithm Approach to Simultaneous Multi-Mission Radar Waveform Design

by

Jason W. Enslin

A Thesis Submitted in Partial Fulfillment of the Requirements for the Degree of Master of Science in Electrical Engineering.

Examining Committee:

Advisor \& Department Head: Dr. Vincent J. Amuso

Member: Dr. Eli Saber

Member: Dr. Sohail Dianat

Department of Electrical Engineering

Kate Gleason College of Engineering

Rochester Institute of Technology

Rochester, New York

August 2007 


\section{Acknowledgements}

I have to begin by acknowledging my family because without them I would not be in the position that I am today. Mom, thank you for doing everything in your power to give me anything that I could ever want or need. Everything that I have accomplished or will accomplish in the future is a testament to you. Gram, the sacrifices you have made for me over the past 22 years mean more to me than I can explain. I want you to know that I appreciate everything and that I have always tried to make you proud. Pop, you have had the most influence on the man that I am today and I know that you are always here with me. Thank you all for making this possible.

Sarah, I can’t say anything more truthful and necessary than thank you for putting up with me over these past few years. We have been through a lot together and I would have been hard pressed to do this without you. Also, I'd be remiss if I didn't take this opportunity to congratulate you on everything you have accomplished as well. You have chased a dream and captured it, and I am tremendously proud of you for that.

Andrea, Avery, Jimy, and Todd, thank you for sharing in our self-proclaimed “misery." It means a lot to have friends who understand exactly what it takes to make it through the things we have. I regret that I didn’t get together with you guys sooner, but I am grateful for this past year. Congratulations to all of you as well, we certainly earned it.

A special thanks has to go to Dr. Amuso for giving me the opportunity to do this work and for being a tremendous advisor since the first day I arrived at RIT. You have provided me with many more opportunities than I could have imagined here and have 
made my college experience truly rewarding. Thank you as well to my advisory board members, Dr. Saber and Dr. Dianat, not only for participating in this work but also for the knowledge and lessons that you have passed along. Finally, thank you to all of the EE department faculty and staff who have helped me along the way. Patti and Florence, the best thing of all about finishing this thesis is that you will no longer have to constantly answer the question, "is he here?" Now you can get at least two more hours worth of other things done each day. In all seriousness though, thank you for all the little things. They have been much appreciated. 


\section{Abstract}

It would be beneficial with today's cluttered electromagnetic spectrum to be able to perform multiple radar missions simultaneously from a single platform. The design of a waveform for this application would greatly benefit the radar community. Radar systems are used to perform many missions, some of which include the detection and tracking of airborne and ground moving targets as well as Synthetic Aperture Radar (SAR) imaging. There are many systems that can operate in multiple modes to perform these missions, although there is no one radar that can simultaneously perform multiple missions using the same waveform [1]. Each mission can be mathematically reduced to an objective or set of objectives that can be used to evaluate their success. These objectives are functions of numerous radar and spatial parameters such as pulse repetition frequency (prf), center frequency, bandwidth, antenna beamwidth, and azimuth look angle, among others. In this thesis, an evolutionary multi-objective optimization technique known as the Strength Pareto Evolutionary Algorithm 2 (SPEA2), developed by Zitzler and Thiele [2], was applied to the simultaneous multi-mission radar waveform design problem. Several of the radar parameters mentioned above were varied to produce diverse waveforms that were manipulated using SPEA2. Due to computational constraints, the problem was approached by using two different scaled down real world scenarios to evaluate the performance of the evolutionary waveform design on a multiobjective moving target indication (MTI) mission and a multi-objective SAR mission, respectively. Multiple experiments showed that SPEA2 can select a set of Pareto optimal 
waveforms that accomplish these multi-objective missions effectively according to the objective functions that were developed for these missions. Finally, a procedure is outlined to combine these multi-objective MTI and SAR missions into one scaled experiment in which a distributed computing environment could be used to provide more computational resources. 


\section{Table of Contents}

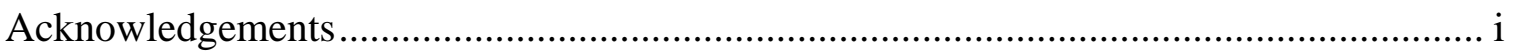

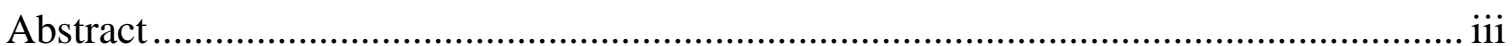

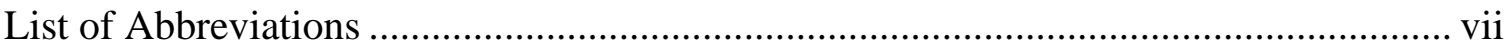

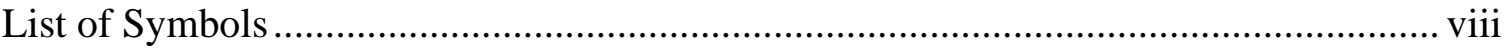

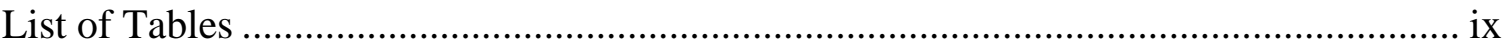

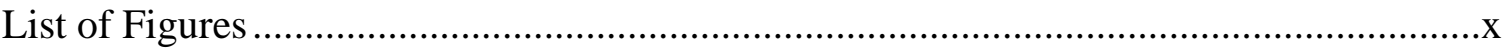

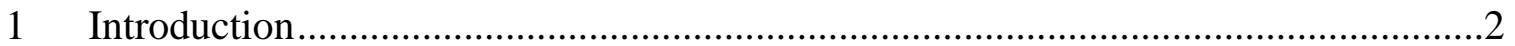

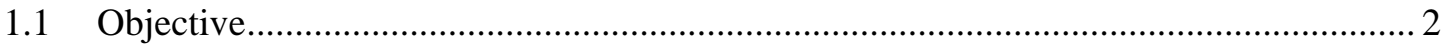

1.2 Literature Review for Multi-Mode/Multi-Mission Waveform Design............................ 4

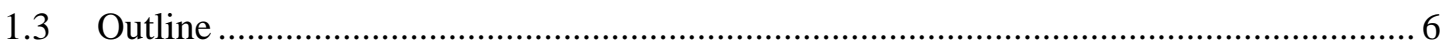

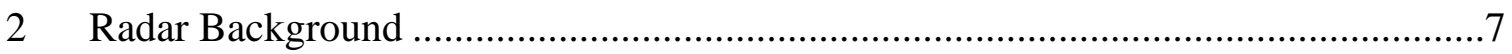

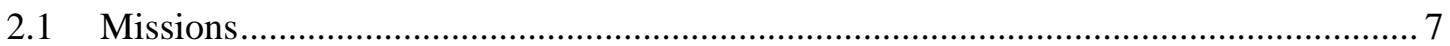

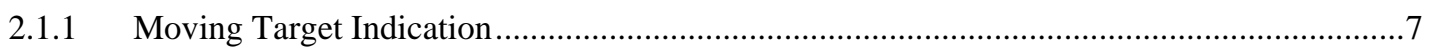

2.1.2 Synthetic Aperture Radar Imaging ...........................................................................12

3 Genetic Algorithms \& Multi-Objective Optimization...........................................17

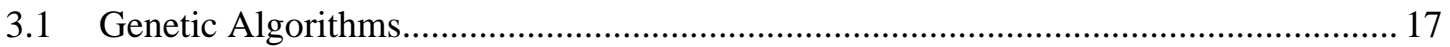

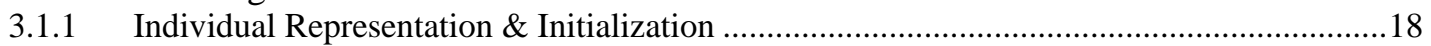

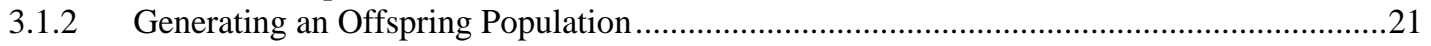

3.2 The Multi-Objective Optimization Problem..................................................................... 24

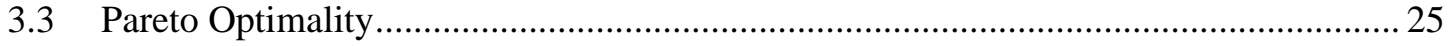

3.4 Evolutionary Multi-Objective Optimization Techniques ..........................................26

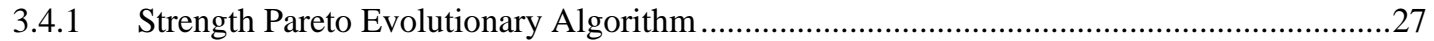

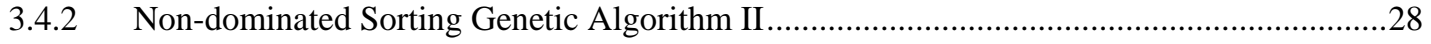

4 The Strength Pareto Evolutionary Algorithm 2 (SPEA2) .......................................30

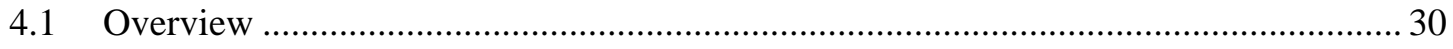

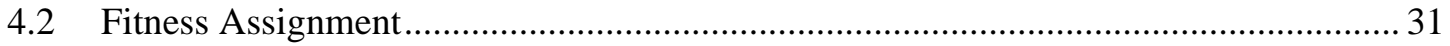

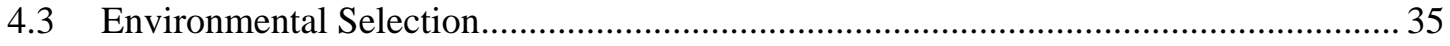

4.4 Verification with the 0/1 Multi-Objective Knapsack Problem ........................................ 37

5 SPEA2 Applied to Simultaneous Multi-Mission Radar Waveform Design..............39

5.1 Waveform Suite Approach .......................................................................................... 39

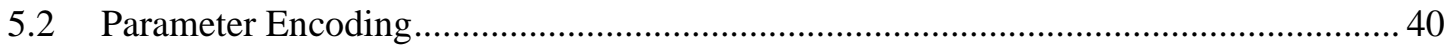

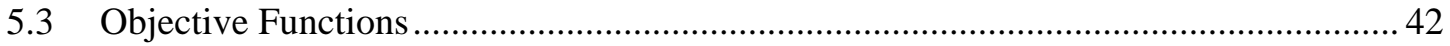


5.3.1 Peak Sidelobe Level \& Integrated Sidelobe Level .........................................................42

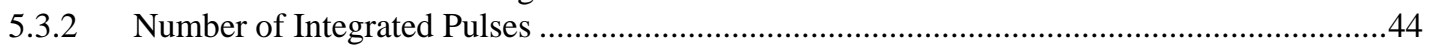

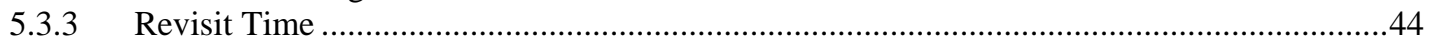

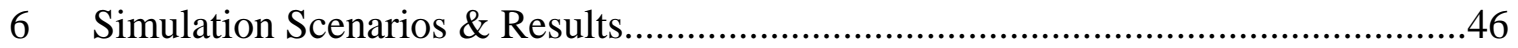

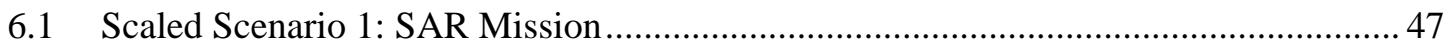

6.1.1 Full Bandwidth, Single Aperture Experiment ..........................................................49

6.1.2 Split Bandwidth, Multiple Sub-Aperture Experiment ...................................................58

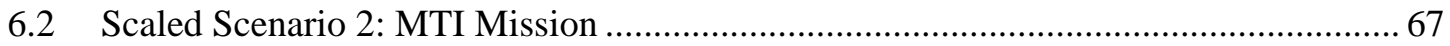

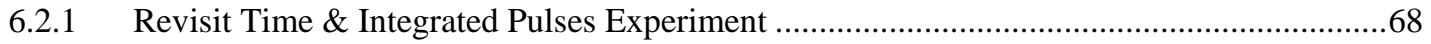

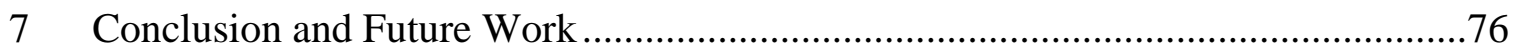

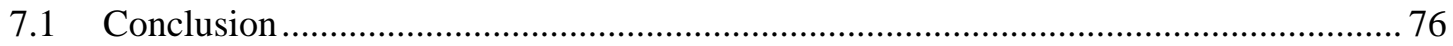

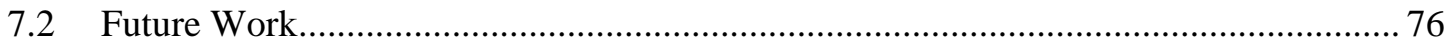

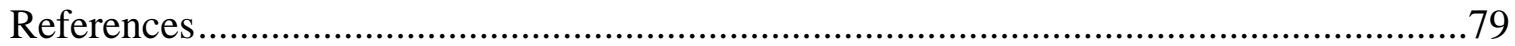




\section{List of Abbreviations}

$\begin{array}{ll}\text { AMTI } & \text { Airborne Moving Target Indication } \\ \text { CPI } & \text { Coherent Processing Interval } \\ \text { FFT } & \text { Fast Fourier Transform } \\ \text { GA } & \text { Genetic Algorithm } \\ \text { GMTI } & \text { Ground Moving Target Indication } \\ \text { GUI } & \text { Graphical User Interface } \\ \text { IFFT } & \text { Inverse Fast Fourier Transform } \\ \text { ISL } & \text { Integrated Sidelobe Level } \\ \text { MOEA } & \text { Multi-Objective Evolutionary Algorithm } \\ \text { MOKP } & \text { Multi-Objective Knapsack Problem } \\ \text { MOP } & \text { Multi-Objective Optimization Problem } \\ \text { MTI } & \text { Moving Target Indication } \\ \text { NGSA-II } & \text { Non-Dominated Sorting Genetic Algorithm II } \\ \text { pdf } & \text { Probability Density Function } \\ \text { PRF } & \text { Pulse Repetition Frequency } \\ \text { PSF } & \text { Point Spread Function } \\ \text { PSL } & \text { Peak Sidelobe Level } \\ \text { PSR } & \text { Point Source Response } \\ \text { ROI } & \text { Region of Interest } \\ \text { SAR } & \text { Synthetic Aperture Radar } \\ \text { SMRW } & \text { Simultaneous Multi-Mission Radar Waveform } \\ \text { SNR } & \text { Signal-to-Noise Ratio } \\ \text { SPEA } & \text { Strength Pareto Evolutionary Algorithm } \\ \text { SPEA2 } & \text { Strength Pareto Evolutionary Algorithm 2 } \\ \text { TS } & \text { Tournament Selection } \\ \text { VEGA } & \text { Vector Evaluated Genetic Algorithm } \\ \text { VPH } & \text { Video Phase History }\end{array}$




\section{List of Symbols}

$\begin{array}{ll}F^{-1} & \text { Inverse Fourier Transform } \\ \in & \text { Belongs To } \\ \forall & \text { For All } \\ \exists & \text { There Exists } \\ \succ & \text { Dominates } \\ \vee & \text { Or } \\ \wedge & \text { And } \\ \Leftrightarrow & \text { If And Only If }\end{array}$




\section{List of Tables}

Table 2-1: Requirements for radar missions of interest [13] ....................................... 10

Table 6-1: Summary of simulation parameters for the full bandwidth, single aperture

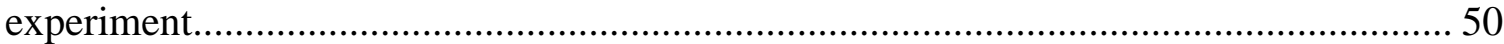

Table 6-2: Summary of the evolution of PSL and ISL values for each full bandwidth,

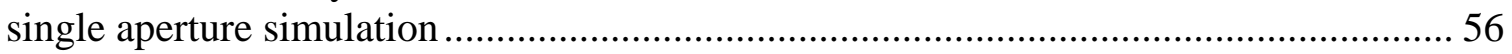

Table 6-3: Summary of simulation parameters for the split bandwidth, multiple sub-

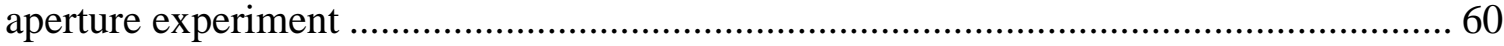

Table 6-4: Summary of the evolution of PSL and ISL values for each split bandwidth,

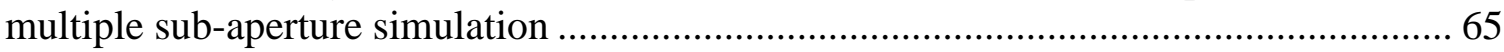

Table 6-5: Single range cell fitness evaluation for revisit time \& integrated pulses experiment.

Table 6-6: Summary of simulation parameters for the revisit time \& integrated pulses experiment. 


\section{List of Figures}

Figure 2-1: A block diagram of a simple MTI radar [15] ........................................... 7

Figure 2-2: Simplified frequency response of a multiple pulse signal [5] ....................... 8

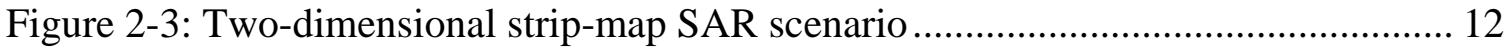

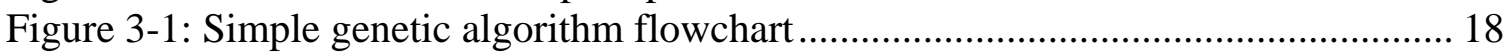

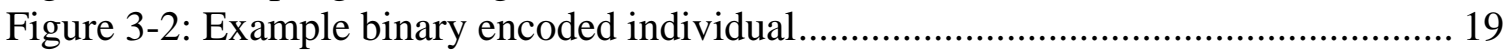

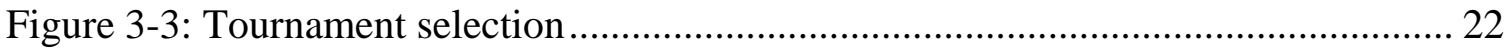

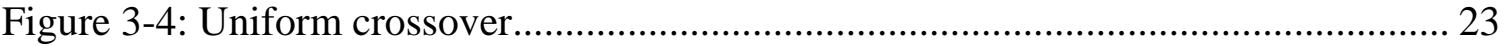

Figure 3-5: A mapping illustration from decision variable space to multi-objective

function space (reproduced from [1] with author's permission) .................................... 24

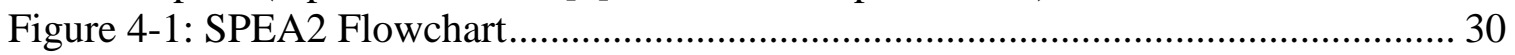

Figure 4-2: Fitness assignment schemes for the same population in SPEA (left) and

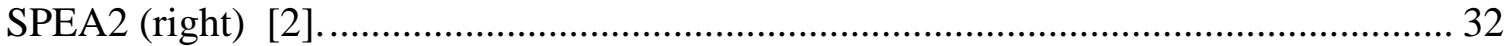

Figure 4-3: Illustration of the SPEA2 archive truncation scheme. $N=4$ is assumed and

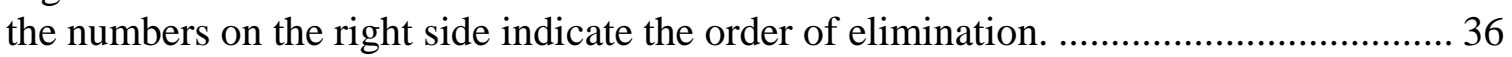

Figure 5-1: Binary encoded waveform suite................................................................. 40

Figure 5-2: RIT Multi-Mission Radar Waveform Tool: Selection of radar parameters GUI

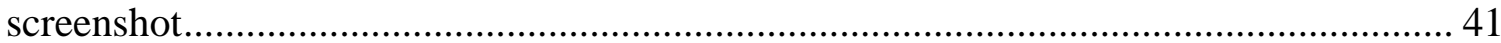

Figure 5-3: PSL SAR objective function. Absolute PSL values greater than $14 \mathrm{~dB}$ result

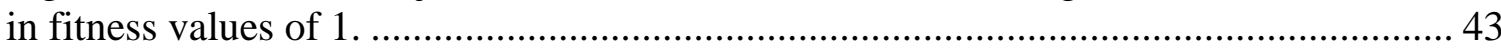

Figure 5-4: ISL SAR objective function. Absolute ISL values greater than $45 \mathrm{~dB}$ result in

fitness values of 1 . Any absolute ISL value less than $20 \mathrm{~dB}$ gives a fitness value of $0 . .43$ Figure 5-5: Number of pulses MTI objective function. The target number of pulses (the number that gives a fitness value of 1 ) is the maximum allowed in the simulation

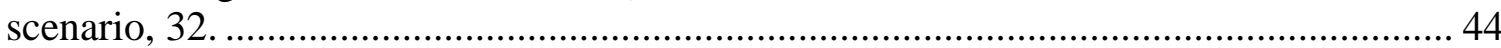

Figure 5-6: Revisit time MTI objective function. The target revisit time is $0.75 \mathrm{~s}$ and results in a fitness value of 1 . The maximum revisit time is $1.5 \mathrm{~s}$ and any value greater

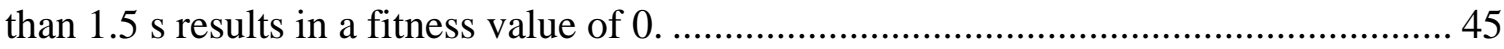

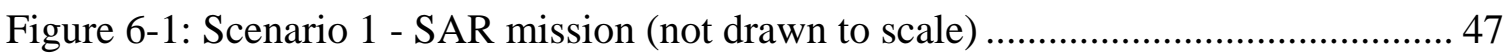

Figure 6-2: Example waveform suite for the full bandwidth, single aperture experiment 49

Figure 6-3: Initial population and final archive population for the full bandwidth, single

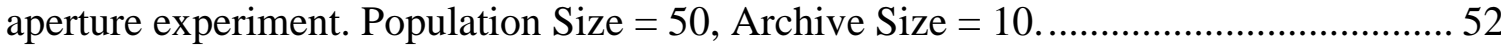
Figure 6-4: Initial (left) and final (right) VPH for the middle cell of the ROI. Population

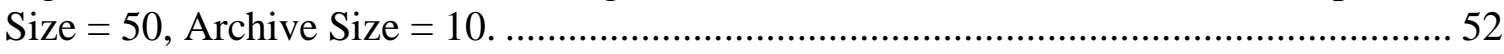

Figure 6-5: Initial (left) and final (right) PSR for the middle cell of the ROI. Population

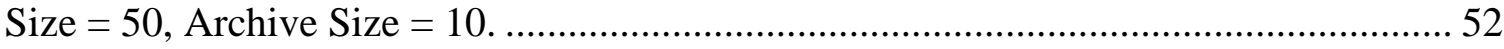

Figure 6-6: Initial population and final archive population for the full bandwidth, single

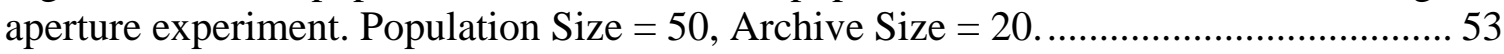
Figure 6-7: Initial (left) and final (right) VPH for the middle cell of the ROI. Population

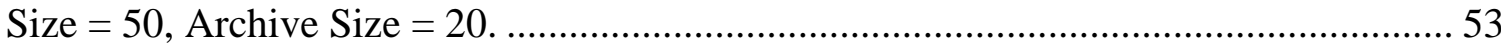


Figure 6-8: Initial (left) and final (right) PSR for the middle cell of the ROI. Population Size $=50$, Archive Size $=20$.

Figure 6-9: Initial population and final archive population for the full bandwidth, single aperture experiment. Population Size $=100$, Archive Size $=10$.

Figure 6-10: Initial (left) and final (right) VPH for the middle cell of the ROI. Population

Size $=100$, Archive Size $=10$. 54

Figure 6-11: Initial (left) and final (right) PSR for the middle cell of the ROI. Population

Size $=100$, Archive Size $=10$. 54

Figure 6-12: Initial population and final archive population for the full bandwidth, single

aperture experiment. Population Size $=100$, Archive Size $=20$. 55 Figure 6-13: Initial (left) and final (right) VPH for the middle cell of the ROI. Population

Size $=100$, Archive Size $=20$. 55 Figure 6-14: Initial (left) and final (right) PSR for the middle cell of the ROI. Population Size $=100$, Archive Size $=20$. 55

Figure 6-15: Initial population and final archive population using five different random seeds for the full bandwidth, single aperture experiment. Population Size $=100$, Archive

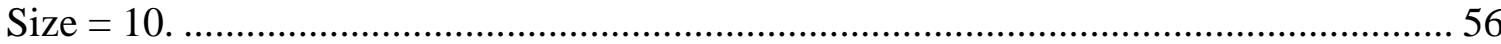
Figure 6-16: Archive population using five different random seeds for the full bandwidth, single aperture experiment. Population Size $=100$, Archive Size $=10$. 57

Figure 6-17: Example waveform suite for the split bandwidth, multiple sub-aperture experiment.. 59

Figure 6-18: Initial population and final archive population for the split bandwidth, multiple sub-aperture experiment. Population Size $=50$, Archive Size $=10$.

Figure 6-19: Initial (left) and final (right) VPH for the middle cell of the ROI. Population Size $=50$, Archive Size $=10$.

Figure 6-20: Initial (left) and final (right) PSR for the middle cell of the ROI. Population

Size $=50$, Archive Size $=10$. 61

Figure 6-21: Initial population and final archive population for the split bandwidth, multiple sub-aperture experiment. Population Size $=50$, Archive Size $=20$. 62 Figure 6-22: Initial (left) and final (right) VPH for the middle cell of the ROI. Population Size $=50$, Archive Size $=20$. 62 Figure 6-23: Initial (left) and final (right) PSR for the middle cell of the ROI. Population Size $=50$, Archive Size $=20$. 62

Figure 6-24: Initial population and final archive population for the split bandwidth, multiple sub-aperture experiment. Population Size $=100$, Archive Size $=10$. 63 Figure 6-25: Initial (left) and final (right) VPH for the middle cell of the ROI. Population Size $=100$, Archive Size $=10$. 63 Figure 6-26: Initial (left) and final (right) PSR for the middle cell of the ROI. Population Size $=100$, Archive Size $=10$. 63

Figure 6-27: Initial population and final archive population for the split bandwidth, multiple sub-aperture experiment. Population Size $=100$, Archive Size $=20$. 64 Figure 6-28: Initial (left) and final (right) VPH for the middle cell of the ROI. Population Size $=100$, Archive Size $=20$. 64 
Figure 6-29: Initial (left) and final (right) PSR for the middle cell of the ROI. Population Size $=100$, Archive Size $=20$.

Figure 6-30: Initial population and final archive population using five different random seeds for the split bandwidth, multiple sub-aperture experiment. Population Size $=100$, Archive Size $=10$.

Figure 6-31: Archive population using five different random seeds for the split bandwidth, multiple sub-aperture experiment. Population Size = 100, Archive Size = 10 .

Figure 6-32: Scenario 2 - MTI mission (not drawn to scale)

Figure 6-33: Example waveform suite for the revisit time \& integrated pulses experiment

Figure 6-34: Initial population and final archive population for the revisit time \& integrated pulses experiment. Population Size $=50$, Archive Size $=10$. 71

Figure 6-35: Initial population and final archive population for the revisit time \& integrated pulses experiment. Population Size $=50$, Archive Size $=20 \ldots \ldots \ldots \ldots \ldots \ldots \ldots \ldots \ldots \ldots \ldots \ldots$ Figure 6-36: Initial population and final archive population for the revisit time \& integrated pulses experiment. Population Size $=100$, Archive Size $=10 \ldots \ldots \ldots \ldots \ldots \ldots \ldots \ldots . . .72$ Figure 6-37: Initial population and final archive population for the revisit time \&

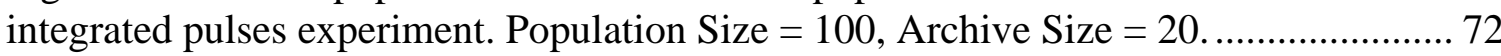
Figure 6-38: Initial population and final archive population for the revisit time \& integrated pulses experiment. Population Size $=200$, Archive Size $=25$. 73

Figure 6-39: Initial population and final archive population using five different random seeds for the revisit time \& integrated pulses experiment. Population Size $=100$, Archive Size $=10$. 


\section{Introduction}

\subsection{Objective}

A radar is an electromagnetic system that operates by transmitting a particular type of waveform and detecting the nature of the returned echo signal from reflecting objects [3]. Radars are utilized for several different "tasks" often referred to as missions. Some of these missions include ground moving target indication (GMTI), airborne moving target indication (AMTI) and synthetic aperture radar (SAR) imaging. Current systems employ mission-specific hardware and waveforms. There are some existing multi-mode radar systems that have the ability to perform different missions [4], although not simultaneously [1]. By reducing the amount of required hardware and containing a large amount of information from a single waveform, a simultaneous multi-mission radar platform would be the most efficient to date.

Different radar missions often have different objectives that may or may not be related. The design of a radar waveform to accomplish such a mission forms a multiobjective optimization problem (MOP). Often times, mission objectives are conflicting and are extremely difficult to effectively optimize. There are numerous radar parameters that must be designed to successfully meet these objectives such as pulse repetition frequency (PRF), center frequency, azimuth look angle, etc. The problem becomes even more complicated when a multi-mission waveform design is considered. The number of objectives increases, and often times, different sets of radar parameters are required for 
each mission. This type of optimization problem where the solution is not intuitive lends itself well to evolutionary techniques.

Genetic algorithms (GAs) are a specific subset of evolutionary computation techniques. They operate on the Darwinian concept of "survival of the fittest." The primary responsibility of the user is to define a fitness evaluation function that can distinguish between "fit" and "unfit" solutions. When this is done effectively, genetic algorithms have the ability to search a vast solution space for an "optimal" solution.

Achieving an "optimal" solution is the goal of every optimization algorithm, but this raises the question of how one defines optimality. This becomes increasingly complicated when a multi-objective scenario is considered. The Strength Pareto Evolutionary Algorithm 2 (SPEA2), developed by Zitzler and Thiele [2], is a multiobjective evolutionary algorithm that optimizes in a Pareto sense. A solution is Pareto optimal (in the context of a maximization problem) if there exists no feasible vector which would increase some criterion without causing a simultaneous decrease in at least one other criterion [5].

In this thesis, SPEA2 is applied to the simultaneous multi-mission radar waveform (SMRW) design problem. Waveform suites attempting to separately perform a multiobjective SAR and a multi-objective MTI mission are generated and evaluated by SPEA2. The success of each mission is determined mathematically using a set of objectives. Objective functions are developed to map mission performance to fitness values that SPEA2 subsequently uses to determine a set of Pareto optimal solutions. Scaled down real-world scenarios were developed in order to evaluate waveform 
performance in a simulated environment. Some real world missions, such as SAR imaging, operate over long periods of time (on the order of 20 minutes) and usually involve a large region of interest (ROI). The scaled scenarios make it possible to evaluate a complete family of Pareto optimal solutions in a reasonable amount of simulation time. The results of numerous simulations demonstrating the fidelity of multi-objective radar waveforms are presented. Finally, a true multi-mission, multi-objective scenario is developed that requires the use of a parallel processing computer cluster in future applications.

\subsection{Literature Review for Multi-Mode/Multi-Mission Waveform Design}

By employing a frequency-diverse waveform generator and a sophisticated digital signal processor, a single radar system capable of performing multiple missions can be developed. These systems require an explicit selection of the radar mode of operation, and then a specific waveform is generated to accomplish the selected mission. Many current radar systems have multi-mode capabilities. Some of these systems are described in [6], [7] and [8]. A perspective on the development of multi-mode radar systems is given by Strong in [9]. Multi-mode capabilities are also being explored in space-based radars, as discussed in [10].

One state-of-the-art multi-mode radar system is described in [4]. The AN/APG-76 multi-mode radar has the unique capability of performing SAR and GMTI missions simultaneously using a multi-aperture antenna. It is important to note that this "simultaneous" capability is the product of essentially combining the results of two 
different radars. Each aperture transmits its own mission-specific waveform to accomplish its task. No multi-mode radar system can currently perform simultaneous missions with a single waveform, which is the aim of this thesis.

There has been some previous research in the area of multi-mode and multimission radar waveform design and diversity. In [11], Mendelson and Ogle describe many of the conflicting parameters for SAR and MTI missions. Their work focuses on a processing technique that improves SAR and MTI mission capability for multi-mode radar systems. Antonik et al. [12] proposed the concept of a frequency diverse array that ignores the traditional separation of the antenna and waveform subsystems which provides additional degrees of freedom for multi-mission applications. Their proposal employs a separate waveform generator for each antenna which is controlled by its own subsystem. Simultaneous multi-mission performance is not addressed by this work.

Amuso et al. [13] performed a systems analysis that demonstrates the difficulty in designing a single radar system that can achieve SAR and MTI missions simultaneously. Interleaved structure for multi-mission waveform design using a simple single-objective genetic algorithm is discussed in this work. Interleaved waveform structure refers to interspersing MTI waveforms with sections of SAR waveforms. This is not true simultaneous multi-mission waveform design, as it is essentially a scheduling problem for MTI and SAR waveforms. Due to the overly simple genetic algorithm and the complexity of the problem, this approach did not produce worthwhile results.

The follow-up work from Amuso et al. employed a multi-objective genetic algorithm known as the Strength Pareto Evolutionary Algorithm (SPEA) to design 
simultaneous multi-mission waveforms [14]. Objective functions were used to evaluate mission performance. Simultaneous multi-mission waveforms were designed for a realworld scenario. Incremental increases in performance with respect to the defined mission objectives were shown for the waveforms designed by SPEA with respect to randomly generated waveforms. This thesis builds on the work presented in [14].

\subsection{Outline}

The organization of this thesis is as follows. Chapter 2 will describe the radar missions of interest and note the difficulty in the design of a single waveform to accomplish these missions. Chapter 3 will discuss genetic algorithms and will form the multi-objective optimization problem. Chapter 4 will describe SPEA2 and note its advantages over the other multi-objective optimization algorithms. Chapter 5 will detail how SPEA2 is applied to the SMRW design problem. It will contain a description of the desired missions to be accomplished as well as the objective functions used to evaluate their success. Chapter 6 will describe the scaled real-world scenarios used for simulation as well as provide the results and analysis of all experiments. Finally, Chapter 7 will give conclusions and future work. 


\section{Radar Background}

\subsection{Missions}

The missions of interest for the SMRW design problem are synthetic aperture radar (SAR) imaging and moving target indication (MTI). These missions are two of the most common in radar application [15]. The following subsections will describe each mission and its relevance to the SMRW design problem.

\subsubsection{Moving Target Indication}

Moving target indication (MTI) is one of the most common missions for a radar to undertake. Separating moving targets from stationary ones is a vital task in many military and security applications. MTI is an extremely important mission in high-quality surveillance radars that operate in areas containing clutter [3]. For a stationary radar, an MTI mission is carried out by measuring the Doppler frequency shift of the received signal. A reference signal at the same frequency as the transmitted signal is utilized for this Doppler shift measurement. A simple MTI radar is shown in Figure 2-1.

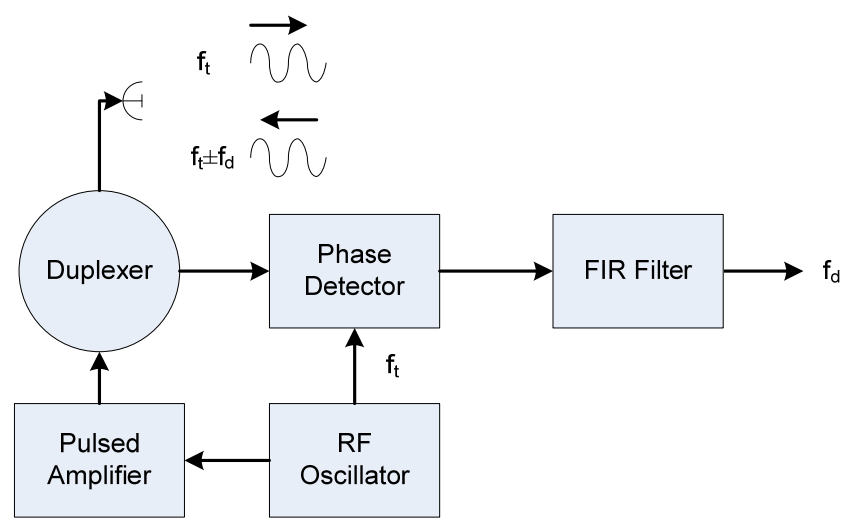

Figure 2-1: A block diagram of a simple MTI radar [15] 
If the transmitted signal is of the form $A_{1} \sin \left(2 \pi f_{t} t\right)$, then the reference signal will be of the same form with possibly a different amplitude, i.e. $A_{2} \sin \left(2 \pi f_{t} t\right)$. The Dopplershifted return echo that is received can then be represented as:

$$
V_{\text {echo }}=A_{3} \sin \left(2 \pi\left(f_{t} \pm f_{d}\right) t-\frac{4 \pi f_{t} R_{0}}{c}\right)
$$

where $f_{d}$ is the Doppler frequency shift, $c$ is the velocity of propagation, and $R_{0}$ is the target range. Only the low frequency $\left(f_{d}\right)$ component of the echo signal is of interest, and must be extracted.

A simplified frequency response of a multiple pulse signal is shown in Figure 2-2. It has zero response at DC, which means that the radar will not detect stationary targets. Also, because of the periodicity of the transmitted signal the response also rejects frequencies in the vicinity of the PRF and its harmonics [3].

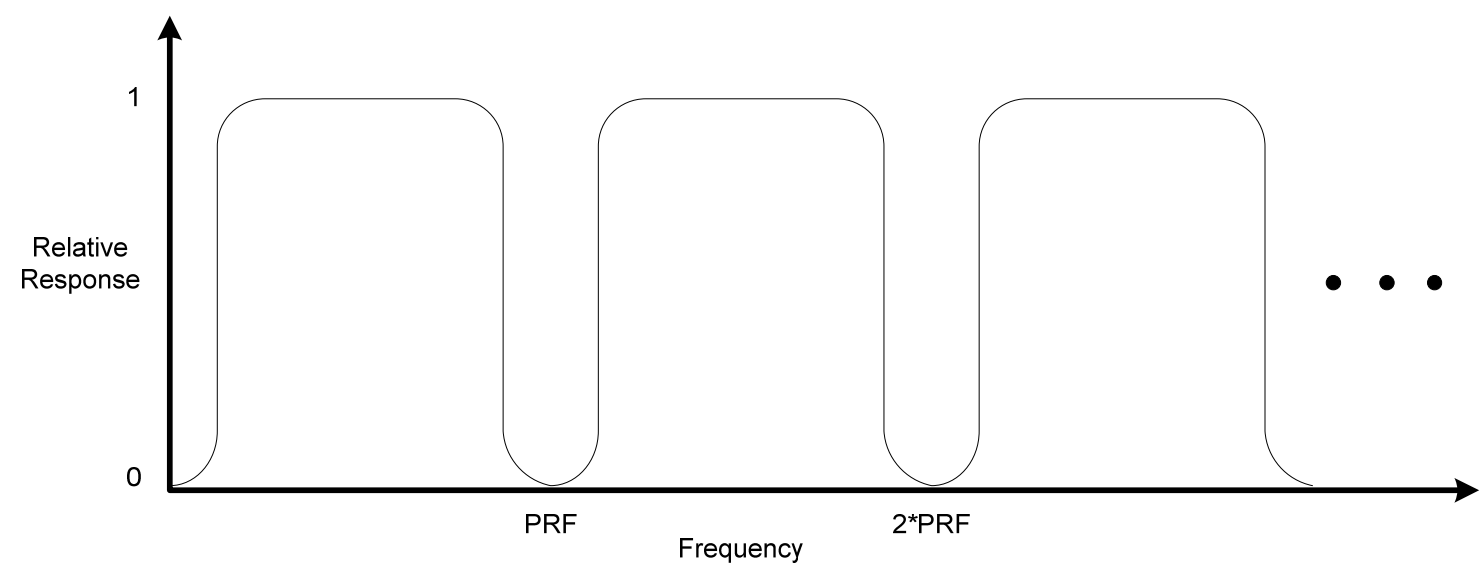

Figure 2-2: Simplified frequency response of a multiple pulse signal Error! Reference source not found.

The nulls in the frequency response are a significant issue with MTI radar systems which are commonly known as "blind speeds.” A blind speed refers to a certain velocity that the 
radar cannot detect, due to its cancellation by the clutter rejection filter. Mathematically, these blind speeds are given as:

$$
v_{n}=\frac{n \lambda(P R F)}{2}
$$

An MTI mission becomes more difficult when the radar platform is in motion. This is the case when the radar is aboard a flying aircraft, as was described in the SAR mission. Clutter is not as easily distinguished when its Doppler frequency shift is no longer equal to zero. However, this effect can be compensated for by one of two methods. The first is to shift the frequency of the oscillator to account for the shift in the clutter Doppler frequency [3]. The Doppler shift of the clutter is determined by the speed of the aircraft and the pointing direction of the antenna. The other compensation method is to redesign the FIR filter to reject the Doppler shift frequencies.

The design of a waveform to accomplish an MTI mission depends on the selection of many parameters. The center frequency $\left(f_{c}\right)$ of the transmitted radar signal will determine the Doppler frequency according to:

$$
f_{d}=\frac{2 v_{r} f_{c}}{c}
$$

where $v_{r}$ is the relative radial velocity of the target with respect to the radar. The velocity range that the radar is interested in detecting depends on what type of mission is to be performed. Also, the range capability of a particular PRF must be considered. A multipletime-around echo is a signal that is received after an interval exceeding the pulse repetition interval. These multiple-time-around echoes result in erroneous range measurements known as range ambiguities [3]. A low PRF reduces the chance receiving 
these multiple-time-around echoes, but as shown by Equation 2-2, it also produces a low first blind speed. A high PRF introduces range ambiguities but also extends the first blind speed so that faster moving targets can be detected. A GMTI mission must be able detect slow moving targets, thus requiring a low PRF to ensure that no targets of interest fall within the range of the blind speeds. In contrast, an AMTI mission must be able to detect much faster moving targets, upwards of $700 \mathrm{~m} / \mathrm{s}$. This requires the use of a medium to high PRF to extend the first blind speed and also to ensure that the target's position is frequently updated so that it is not lost. Table 2-1 [13] gives a summary of the conflicting parameters needed to accomplish AMTI, GMTI, and SAR missions. The table contains two parameters not previously discussed, dwell time and revisit time. Dwell time refers to the total amount of time that a target is illuminated during a mission while revisit time is the desired time between illuminations of a target during the mission.

\begin{tabular}{|c|c|c|c|}
\hline Mission & AMTI & GMTI & Stripmap SAR \\
\hline Dwell Time & $\begin{array}{c}\text { Short } \\
(100 \mathrm{~ms})\end{array}$ & $\begin{array}{c}\text { Moderate } \\
(0.5 \mathrm{~s})\end{array}$ & $\begin{array}{c}\text { Very Long } \\
(20 \mathrm{~min})\end{array}$ \\
\hline Revisit Time & $\begin{array}{c}\text { Short } \\
(\sim 10 \mathrm{~s})\end{array}$ & $\begin{array}{c}\text { Moderate } \\
(30 \mathrm{~s})\end{array}$ & Long \\
\hline PRF & $\begin{array}{c}\text { Medium } \\
(>1 \mathrm{KHz})\end{array}$ & $\begin{array}{c}\text { Low } \\
(>200 \mathrm{~Hz})\end{array}$ & $\begin{array}{c}\text { Low } \\
(\sim 250 \mathrm{~Hz})\end{array}$ \\
\hline Bandwidth & $\begin{array}{c}\text { Narrow } \\
(1 \mathrm{MHz})\end{array}$ & $\begin{array}{c}\text { Moderate } \\
(5 \mathrm{MHz})\end{array}$ & $\begin{array}{c}\text { Wide } \\
(30 \mathrm{MHz})\end{array}$ \\
\hline Beamwidth & Narrow & Narrow & Wide \\
\hline
\end{tabular}

Table 2-1: Requirements for radar missions of interest [13]

When multiple pulses are transmitted from the radar, there are more opportunities to detect and extract information from the echo signal. The likelihood that a target is detected by a radar can be described by the following expression, known as the probability of detection $\left(P_{d}\right)$. 


$$
P_{d}=\int_{V_{t}}^{\infty} p_{s n}(v) d v
$$

$V_{t}$ is the voltage threshold for detection and $p_{s n}$ is the signal-plus-noise probability density function (pdf) which depends on the signal-to-noise ratio as well as the signal and noise statistics. $P_{d}$ is a monotonic-increasing function of signal-to-noise ratio (SNR) for a given threshold [15]. When many pulses are returned from a target, they can be summed to improve the probability of detection. This process is called pulse integration. Pulse integration can be done in two ways, before the threshold detection process (pre-detection or coherent integration) or after the threshold detection (post-detection or non-coherent integration) [3]. For ideal pre-detection, the SNR is improved by a factor equal to the number of integrated pulses $(M)$. For ideal post-detection, the SNR is improved by a factor approximately equal to $\sqrt{M}$ as $M$ becomes large [15]. Therefore, the number of pulses that a radar emits upon each target is a critical factor for the detection of that target.

MTI missions are a critical radar task, especially in military and air traffic control applications [8], [9]. The detection and tracking of moving targets has become increasingly important over the years [9]. Also imperative to military applications is the ability to image an area. In some instances it may be critical to obtain images in high resolution while others might require a system that can image through foliage. SAR imaging can perform each of these tasks, among others, and thus is an important mission for a radar to be able to accomplish. 


\subsubsection{Synthetic Aperture Radar Imaging}

Synthetic aperture radar takes advantage of the motion of its carrier vehicle to produce a high cross-range resolution. Effectively, the motion causes the synthesis of a large antenna aperture. SAR achieves its high cross-range resolution by using the motion of the vehicle to generate the antenna aperture sequentially rather than simultaneously as with a conventional array antenna [3].

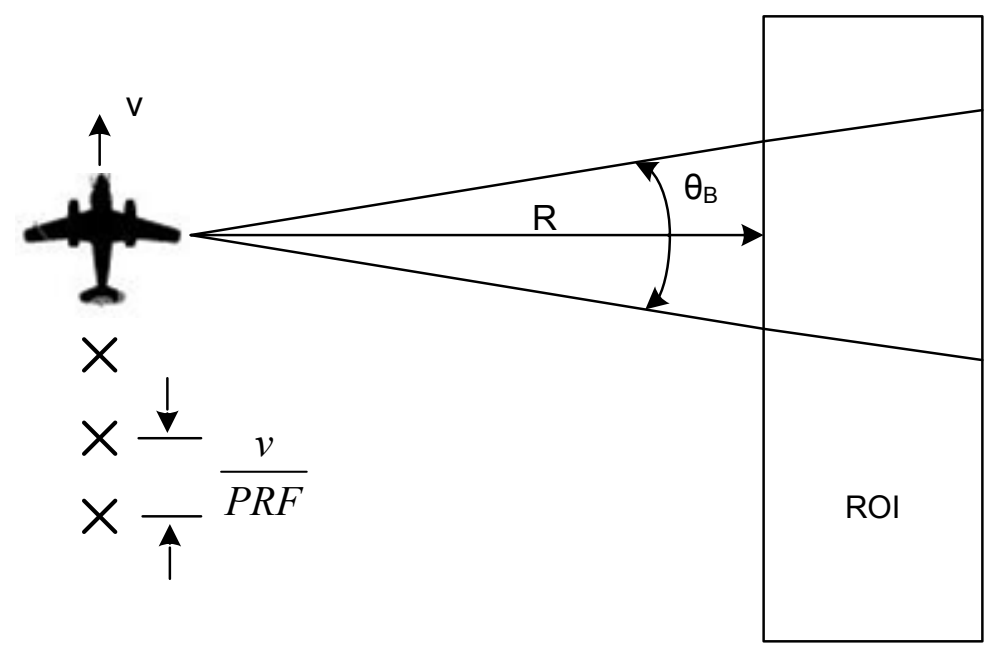

Figure 2-3: Two-dimensional strip-map SAR scenario

Figure 2-3 depicts a two-dimensional SAR scenario. The antenna is positioned to transmit perpendicular to the direction of motion, which is referred to as sidelooking radar or a strip-map SAR configuration. The X's represent the positions at which a pulse is emitted. ROI denotes the region of interest, $\theta_{B}$ is the antenna beamwidth, $v$ is the velocity of the aircraft, $R$ is the range, and PRF is the pulse repetition frequency. The effective aperture length $\left(L_{e f f}\right)$ is equal to $R \theta_{B}$.

There are two fundamental limits to the effective aperture length that can be created by SAR. The first is that $L_{\text {eff }}$ can be no larger than the width of the illuminated region 
$\left(L_{\text {eff }} \leq R \theta_{B}\right.$ ). The second constraint is that the aperture size must be restricted so that the phase front can be considered as a plane wave, also known as the far field of the array [3]. The far field of an antenna is the minimum distance that the rays originating from a radiating source may be considered parallel to each other at the target [3]. This far field condition results in what is known as unfocused SAR. In unfocused SAR, the best achievable resolution is a function of the square root of the range. However, if a correction factor is applied that takes into account the curvature of the spherical wavefront, the resolution can become independent of range. This phase correction factor is defined as follows:

$$
\Delta \varphi=\frac{2 \pi x^{2}}{\lambda R}
$$

where $\lambda$ is the wavelength and $x$ is the distance from the center of the synthetic aperture. With this correction, all the received echo signals at range $R$ are in phase and the SAR is considered to be focused at this range. The cross-range resolution of the focused SAR is

$$
\delta_{c r}=\frac{D}{2}
$$

where $D$ is the size of the actual antenna. The down-range resolution is strictly a function of the bandwidth $(B)$ of the radar signal and can be approximated as follows [16]:

$$
\delta_{d r}=\frac{c}{2 B}
$$

where $c$ is the wave propagation speed.

A SAR mission imposes constraints upon the PRF, just as an MTI mission does. For SAR imaging, the PRF must be low enough to avoid range ambiguities and high 
enough to avoid angle ambiguities (image fold-over) that result from too large a spacing between the synthetic array elements. The distance traveled by the radar between pulse transmissions should be less than half a wavelength [3]. This constraint places the first grating lobe $\left(\theta_{g}\right)$ of the synthetic array at

$$
\theta_{g}=\frac{\lambda(P R F)}{2 v}
$$

Grating lobes are other maxima in a radiation pattern that are not the desired main beam. They have the effect of producing undesired echo signals and thus usually should be minimized or avoided [3]. It is known that the first null of the physical antenna $\left(\theta_{n}\right)$ is located at

$$
\theta_{n}=\frac{\lambda}{D}
$$

Since $\theta_{g} \geq \theta_{n}$ to avoid grating lobes, and combining Equations 2-6, 2-8 \& 2-9, the lower limit on the PRF is defined to be:

$$
P R F \geq \frac{v}{\delta_{c r}}
$$

The upper limit on the PRF is imposed by the unambiguous range $\left(R_{u}\right)$ constraint that was previously discussed in Section 2.1.1. The PRF can not be greater than the amount of time that it takes for a radar signal to travel to the target and back. Imposing this constraint and combining with Equation 2-10 yields the range of PRF's that can be selected for a SAR mission. 


$$
\frac{v}{\delta_{c r}} \leq P R F \leq \frac{c}{2 R_{u}}
$$

One technique to generate a SAR image is by first collecting a video phase history (VPH). The cross-range dimension of the VPH contains spatial information while the down-range dimension contains frequency information. Consider the problem of imaging a region of interest (ROI) as depicted in Figure 2-3. The ground can be considered the "target” in a terrain imaging application. As the radar travels along its path of motion, it receives echo signals from the ground that differ in phase. This phase information is stored as it is received at each position and effectively comprises the spatial information in the cross-range dimension. It can be thought of as a discrete signal in the spatial frequency domain, sampled at a rate equal to the velocity of the aircraft divided by the PRF. The down-range dimension of the VPH is filled by indicating which part of the frequency spectrum that the received signal belongs to. As noted by Equation 2-7, the down-range resolution is determined by the bandwidth of the transmitted signal - i.e. narrow pulses give high resolution. Narrow pulses are short in time which results in a large frequency spectrum. This yields more bandwidth, and thus increased down-range resolution.

The VPH generated by a SAR system can be represented in the frequency ( $\omega$ down-range) and spatial frequency domain ( $k_{u}$ - cross-range) by the following equation:

$$
S\left(\omega, k_{u}\right)=P(\omega) \sum_{n} A_{n}\left(\omega, k_{u}\right) A\left(\omega, k_{u}\right) \exp \left(-j \sqrt{4 k^{2}-k_{u}^{2}} x_{n}-j k_{u} y_{n}\right)
$$

where $P(\omega)$ is the Fourier transform of the transmitted radar signal, $A_{n}$ is the is Doppler amplitude pattern for the $\mathrm{n}^{\text {th }}$ target, $A$ is the Doppler transmit-receive mode radar 
amplitude pattern, $k$ is the wavenumber, $x_{n}$ is the down-range of the $\mathrm{n}^{\text {th }}$ target, and $y_{n}$ is the cross-range of the $\mathrm{n}^{\text {th }}$ target. The SAR image is then reconstructed using matchedfiltered inversion as shown here:

$$
F\left[k_{x}\left(\omega, k_{u}\right), k_{y}\left(\omega, k_{u}\right)\right]=P^{*}(\omega) A^{*}\left(\omega, k_{u}\right) S\left(\omega, k_{u}\right)
$$

The reconstructed image in the spatial domain is then obtained by applying a 2D inverse Fourier transform as follows:

$$
f(x, y)=\sum_{n} f_{n}\left(x-x_{n}, y-y_{n}\right)
$$

where

$$
f_{n}(x, y)=F_{\left(k_{x}, k_{y}\right)}^{-1}\left(\left|P(\omega) A\left(\omega, k_{u}\right)\right|^{2} A_{n}\left(\omega, k_{u}\right)\right)
$$

The $\mathrm{n}^{\text {th }}$ target function $f_{n}(x, y)$ is the point spread function (PSF) of the SAR imaging system [16] and will be utilized to determine the quality of the SAR image in the experiments to follow.

It has been shown that like an MTI mission, a SAR mission requires the distinction of numerous parameters [1], [13]. The bandwidth of the transmitted signal is directly related to the achievable down-range resolution of the SAR image, while the cross-range resolution has an effect on the selection of PRF. It is of course desirable to achieve the highest resolution possible, but constraints are imposed as shown in Equation 2-11 and also by other missions. The selection of the parameters such as PRF, center frequency, number of pulses, bandwidth, etc. is where the challenge lies for multimission waveform design. 


\section{Genetic Algorithms \& Multi-Objective Optimization}

\subsection{Genetic Algorithms}

Evolutionary algorithms use the concept of "survival of the fittest" and mathematically implement this concept into an algorithm in order to produce a generic stochastic approach to solving single or multi-objective optimization problems [17]. Genetic algorithms are a specific subset of evolutionary algorithms that consist of numerically encoded population members, commonly referred to as individuals ${ }^{1}$. Each individual is represented by a number of genes that are grouped together to form chromosomes. The information stored in the chromosomes is then extracted and evaluated mathematically by an application-specific fitness function. This fitness measure is then used to rank individuals within the population. At this point, a selection process is applied to determine which members will pass to the next generation and be permitted to produce offspring. This general process is repeated until the desired number of generations is reached. A simple flowchart describing a basic genetic algorithm is shown in Figure 3-1.

\footnotetext{
${ }^{1}$ It should be known that throughout this thesis, the terms “individual," "population member," and "solution" are used interchangeably.
} 


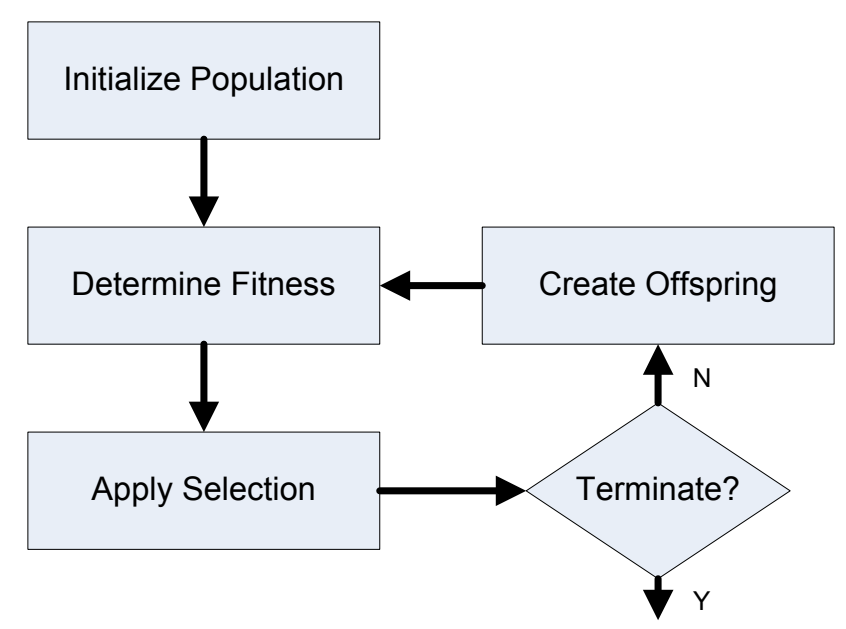

Figure 3-1: Simple genetic algorithm flowchart

Genetic algorithms have the ability to explore many solutions throughout a vast solution space. A genetic algorithm is a robust, stochastic search technique that has the ability to evaluate a group of solutions in parallel and continuously refine them [18]. This is the main characteristic of GAs that has made them so popular in a wide variety of applications such as engineering [18] [19], finance [20], and computer science [21] among others. The following sub-sections will describe each element of a genetic algorithm in detail so that the reader is familiar with GA terminology and concepts before SPEA2 is discussed.

\subsubsection{Individual Representation \& Initialization}

To begin the discussion on the functionality of a typical GA, the numerical representation of a single population member (individual) must first be addressed. From an algorithmic point of view, the individual is nothing more than an encoded solution to a mathematical problem [1]. Binary encoding of individuals was used for the radar application addressed by this thesis. As will be shown in Section 3.1.2, genetic operators 
such as crossover and mutation are easily applied when binary encoding is used. An example of a binary encoded individual is shown in Figure 3-2.

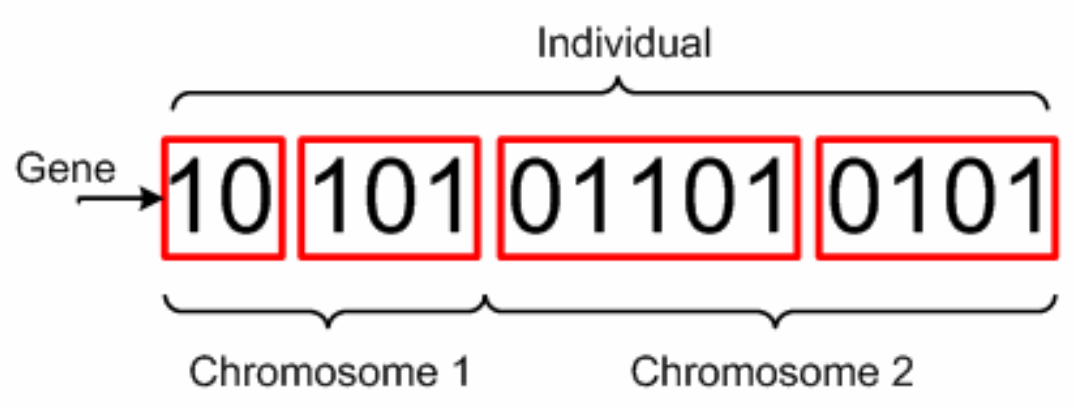

Figure 3-2: Example binary encoded individual

Each binary encoded gene represents a physical characteristic of the individual. A collection of genes is known as a chromosome. As can be seen in Figure 3-2, both genes and chromosomes can be of different lengths because there may be more bits needed to encode one physical characteristic than another. The chromosomes contain the encoded information that distinguishes each individual. The actual encoded values represented by each gene are referred to as alleles [14].

To illustrate the aforementioned concepts, consider a human individual example, as outlined in [1]. The nucleus of most human cells is comprised of two sets of chromosomes, one coming from each parent. This results in 46 total chromosomes. These chromosomes contain specific information about the individual. For an example, take eye color. The gene that determines eye color is found in the same chromosome and position for all humans. The value located in that particular position determines the actual color of the individual's eyes. If there were four possible choices for eye color, two bits would be 
used for the encoding. For instance, say 00 - blue, 01 - brown, 10 - green, 11 - hazel. Therefore, if the eye color gene was 01, the allele would be brown.

The genetic algorithm begins with the generation of an initial population $\left(\mathrm{P}_{0}\right)$ consisting of $\mathrm{N}$ individuals. This is usually accomplished by using a random number generator. Once $\mathrm{P}_{0}$ is generated, each individual is evaluated by an application-specific fitness function. It is at this point where the individual is decoded and mathematically evaluated. This is the most crucial part of any GA. A fitness function that does not effectively represent the desired characteristics for a solution will yield a poor result. The fitness function for the SMRW design problem will be described in the following chapter. For this discussion, it is not necessary to elaborate on how the fitness of an individual is determined.

At this point, a common step is to rank the population members according to their fitness. The ranking establishes order within the population. Depending on the fitness function, this ranking can be done in ascending or descending order. Typically, most genetic algorithms aim to increase fitness so descending ranking would be appropriate in this case. This is usually the point in a GA where a check is performed to determine if the maximum number of generations has been reached. If it has, then the algorithm terminates and the current population $\left(\mathrm{P}_{\mathrm{i}}\right)$ contains the possible solutions to the problem. Because of the ranking process, the most "fit" individual is the first individual in the solution population. 


\subsubsection{Generating an Offspring Population}

If the maximum number of generations has not been reached, the algorithm continues and the offspring creation process is commenced. At this point, many GAs employ a technique known as elitism. To ensure that the best solutions survive to the next generation, $X$ number of the most "fit" individuals are passed directly to the next generation population $\left(\mathrm{P}_{\mathrm{i}+1}\right)$. These $X$ population numbers are usually referred to as the "elite" members. This elitism technique has been shown to increase the performance of GAs both mathematically [22] and empirically [23].

Next, mating pairs (or "parents") must be selected from the population. There are many different techniques that have been developed for this task. Some of these methods include roulette wheel selection, rank selection, tournament selection and basic random selection. Intuitively, a selection scheme that produces a balance between favoring the traits of the best members and providing diversity by allowing many population members to participate would most likely result in an effective search. For this reason, the mating selection process that was chosen for the problem addressed by this thesis is tournament selection (TS). TS provides the diversity that is needed to keep the population from becoming stagnant by allowing all members to participate but it also favors the individuals who possess higher fitness. The advantageous traits of tournament selection including its speed and effectiveness are documented in [23]. TS begins by randomly selecting four individuals from the current population, $\mathrm{P}_{\mathrm{i}}$. The four individuals are grouped in pairs of two, and their fitness values are compared within these groups. The two individuals with the higher fitness are selected to be the first mating pair. This 
process is repeated until the desired amount of mating pairs is achieved. A pictorial representation of tournament selection is shown in Figure 3-3.

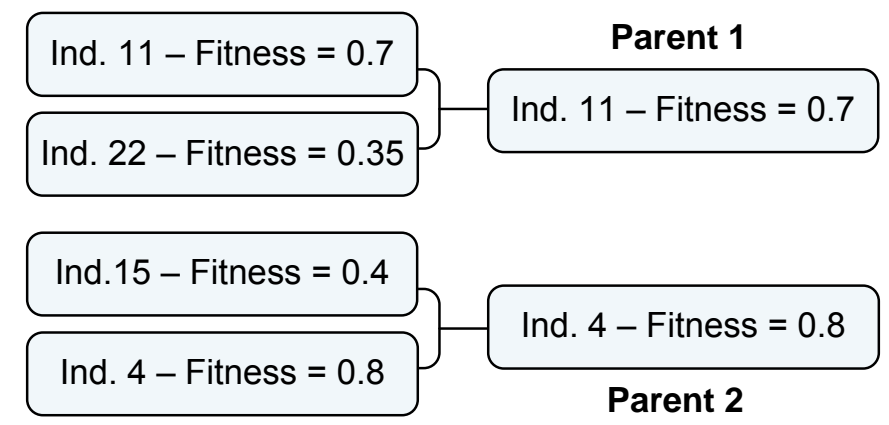

Figure 3-3: Tournament selection

Once the parents are selected, a crossover operation occurs to create offspring. There are also several crossover methods that can be implemented. Some of these include single-point crossover, two-point crossover, and uniform crossover. Each technique is inherently similar, as they all accomplish the same task of swapping genes between the two parents. However, there are constraints to the single-point and two-point crossover methods. In each technique, the crossover point must be selected at the beginning of a gene. This constraint is imposed to ensure that the offspring population is a combination of the physical characteristics of their parents. If this constraint was not met, the gene that was split by the crossover would essentially be a mutation, meaning it may not be a trait of either of the parents. Uniform crossover was chosen because it does not impose the crossover point constraint. Uniform crossover selects a random number of genes to be swapped between the two parents to create two offspring. Uniform crossover is illustrated in Figure 3-4 where $G_{x y}$ represents a binary gene. As can be seen in the figure, genes simply have the ability to change values in uniform crossover. There is no chance of 
shifting a gene's position within a chromosome or changing the gene length. Any crossover operation is usually subject to a crossover probability. This probability determines the chance that two parent individuals will mate to produce offspring or simply copy themselves as the offspring.

\begin{tabular}{|l|l|l|}
\hline Parent 1 & $\mathrm{G}_{11} \mathrm{G}_{12} \mathrm{G}_{13} \mathrm{G}_{14} \mathrm{G}_{15} \mathrm{G}_{16} \mathrm{G}_{17} \mathrm{G}_{18} \mathrm{G}_{19}$ \\
\hline Parent 2 & $\mathrm{G}_{21} \mathrm{G}_{22} \mathrm{G}_{23} \mathrm{G}_{24} \mathrm{G}_{25} \mathrm{G}_{26} \mathrm{G}_{27} \mathrm{G}_{28} \mathrm{G}_{29}$ \\
\hline Offspring 1 & $\mathrm{G}_{11} \mathrm{G}_{22} \mathrm{G}_{13} \mathrm{G}_{24} \mathrm{G}_{15} \mathrm{G}_{16} \mathrm{G}_{27} \mathrm{G}_{18} \mathrm{G}_{19}$ \\
\hline Offspring 2 & $\mathrm{G}_{21} \mathrm{G}_{12} \mathrm{G}_{23} \mathrm{G}_{14} \mathrm{G}_{25} \mathrm{G}_{26} \mathrm{G}_{17} \mathrm{G}_{28} \mathrm{G}_{29}$ \\
\hline
\end{tabular}

Figure 3-4: Uniform crossover

The number of offspring that are generated is algorithm specific. Generally, the population size remains constant; therefore the number of offspring equals the population size minus the elite population that was passed to the next generation. Before the offspring proceed to the next generation, a mutation operator is often invoked to introduce more variety in the search space. Binary mutation simply selects a random number of bits that comprise the offspring's genes to invert. A mutation probability is used to determine how many bits are mutated. Typically, this is a small probability because mutating many bits tends to make the GA perform more like a random search technique [24]. At this point, the offspring population is combined with the elite population and $\mathrm{P}_{\mathrm{i}+1}$ is complete. The $\mathrm{P}_{\mathrm{i}+1}$ population is then passed to the fitness evaluation function and the process is repeated until the desired number of generations is reached. 


\subsection{The Multi-Objective Optimization Problem}

The Multi-objective Optimization Problem (MOP) can be defined as the problem of finding a vector of decision variables which satisfies constraints and optimizes a vector function whose elements represent the objective functions [25]. These objective functions are often in conflict with one another and thus an ideal solution vector often times does not exist. Pareto optimality defines a criterion to estimate the ideal solution and will be discussed in the following section. First, the MOP and the ideal solution vector will be defined mathematically.

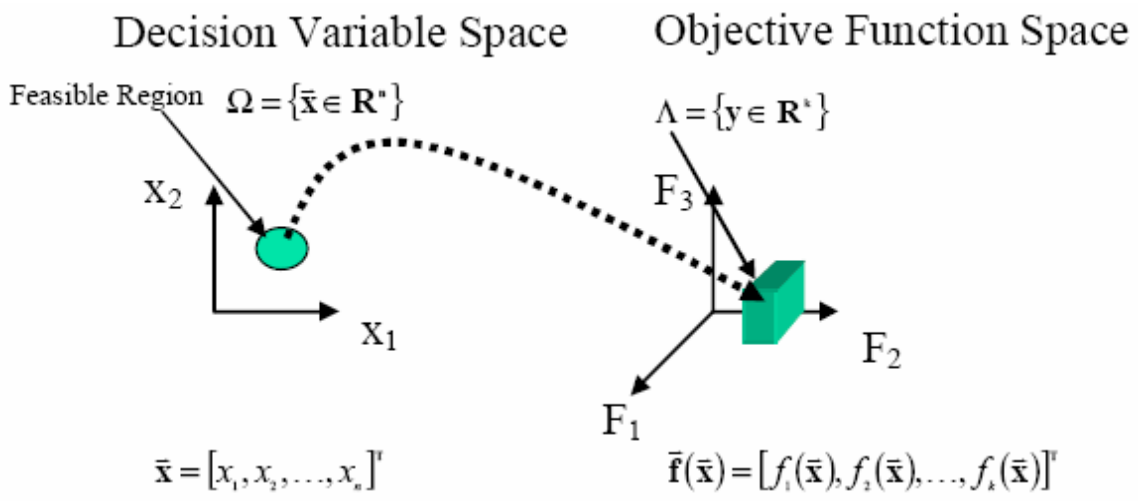

Figure 3-5: A mapping illustration from decision variable space to multi-objective function space (reproduced from [1] with author's permission)

Figure 3-5 illustrates the mapping of a solution (decision space) to a multiobjective function space [1]. The MOP can be defined as follows:

Find a vector

$$
\vec{x}^{*}=\left[x_{1}{ }^{*}, x_{2}{ }^{*}, \ldots x_{n}{ }^{*}\right]^{T}
$$

which satisfies the $m$ inequality constraints

$$
g_{i}(x) \geq 0 \text { for } i=1,2, \ldots m
$$


and the $p$ equality constraints

$$
h_{i}(x)=0 \text { for } i=1,2, \ldots p
$$

as well as optimizes the vector function

$$
\vec{f}(\vec{x})=\left[f_{1}(\vec{x}), f_{2}(\vec{x}), \ldots f_{k}(\vec{x})\right]^{T}
$$

In the context of the SMRW design problem, Equation 3-4 will be used to contain the objective functions that map to the particular mission requirements. Now, let

$$
\vec{x}^{O(i)}=\left[x_{1}{ }^{O(i)}, x_{2}{ }^{O(i)}, \ldots x_{n}{ }^{O(i)}\right]^{T}
$$

be a vector that optimizes the $i$ th objective function. The vector

$$
\vec{x}^{O(i)} \in \Omega
$$

is such that

$$
f_{i}\left(\vec{x}^{O(i)}\right)=\underset{x \in \Omega}{\text { opt }} f_{i}(\vec{x})
$$

Thus the vector

$$
\vec{f}=\left[f_{1}^{O}, f_{2}^{O}, \ldots f_{k}^{O}\right]^{T}
$$

is ideal for a MOP. The point in $\mathbf{R}^{\mathrm{n}}$ space that determines this vector shown in Equation 3-8 is referred to as the ideal vector [1].

\subsection{Pareto Optimality}

When multiple, possibly conflicting, objectives are considered a compromise must be attained in the optimization process. Pareto optimality, first introduced by Edgeworth in 1881 and then generalized by Pareto in 1896, addresses this trade-off problem. A 
solution is Pareto optimal if there exists no feasible vector which would increase some criterion (in a maximization problem) without causing a simultaneous decrease in at least one other criterion [5]. To understand Pareto optimality, the concept of dominance with respect to decision vectors must be defined. Consider two decision vectors, denoted $\vec{v}_{1}$ and $\vec{v}_{2}$ which belong to the solution set $\Omega$. In context of a maximization problem, $\vec{v}_{1}$ is said to dominate $\vec{v}_{2}$ if and only if

$$
\forall i \in\{1,2, \ldots n\}: f_{i}\left(\vec{v}_{1}\right) \geq f_{i}\left(\vec{v}_{2}\right) \wedge \exists j \in\{1,2, \ldots n\}: f_{j}\left(\vec{v}_{1}\right)>f_{j}\left(\vec{v}_{2}\right)
$$

All decision vectors which are not dominated by any other decision vector of the solution set are referred to as non-dominated [5]. Non-dominated decision vectors are considered to be Pareto optimal. A collection of these non-dominated vectors is referred to as a Pareto front. In the context of the MOP previously described, a vector $\vec{x}^{*} \in \Omega$ is Pareto optimal if for every $\vec{x} \in \Omega$ and $I=\{1,2, \ldots k\}$ either

$$
\forall_{i \in I}\left(f_{i}(\vec{x})=f_{i}\left(\vec{x}^{*}\right)\right)
$$

or there is at least one $i \in I$ such that

$$
f_{i}(\vec{x})<f_{i}\left(\vec{x}^{*}\right)
$$

\subsection{Evolutionary Multi-Objective Optimization Techniques}

In the late 1960's, genetic algorithms were beginning to be applied in singleobjective optimization problems. The first true multi-objective evolutionary algorithm (MOEA) is generally attributed to J. David Schaffer for his work on the Vector Evaluated 
Genetic Algorithm (VEGA) [26] in the mid 1980's. Since then, many different MOEAs have been proposed, with only a certain few achieving noted success. Most successful MOEAs incorporated David E. Goldberg's ideas [27] on the use of non-dominated ranking and selection to help guide solutions toward the Pareto optimal front. An excellent review of the history of MOEAs is presented by Carlos A. Coello Coello in [28]. An overview of two MOEAs noted in his article is presented in the following subsections as a precursor to Chapter 4, which describes the Strength Pareto Evolutionary Algorithm 2.

\subsubsection{Strength Pareto Evolutionary Algorithm}

Zitzler and Thiele introduced SPEA in the late 1990's [5] and it quickly became one of the most popular MOEAs because of its incorporation of elitism. SPEA utilizes an archive of non-dominated population members which make up the elite population. Once a member enters the archive, it is guaranteed to remain there until another solution comes along that dominates it. Each individual in the archive is assigned a strength value that is proportional to the number of solutions it dominates. Then, each individual in the population is assigned a fitness value based on the strengths of the archive members that dominate them. The archive size is limited in SPEA; if the archive grows larger than a predefined limit a clustering technique is used that removes solutions that are located close to one another in an attempt to preserve the characteristics of the Pareto front. Mating pairs are chosen by using tournament selection on both the archive and the population, which was another novel concept implemented by SPEA. 
SPEA was shown to perform well on a variety of multi-objective problems [5], [29] and many of its characteristics were adopted by other techniques [30], [31]. However, as [31] would point out, SPEA contained some deficiencies that limited its effectiveness in several instances. These deficiencies are addressed by SPEA's authors in the development of SPEA2.

\subsubsection{Non-dominated Sorting Genetic Algorithm II}

Kalyanmoy Deb et al. introduced the Non-dominated Sorting Genetic Algorithm II in 2000 and published it in 2002 [31]. This algorithm implements a fast population ranking scheme which reduces its computational complexity compared to most other MOEAs. Also, rather than employing the fitness sharing technique proposed in its first version [32] to promote diversity in the population, NSGA-II uses a crowdedcomparison operator to accomplish this task. Fitness sharing requires a user-defined sharing parameter, often denoted $\sigma_{\text {share }}$, which determines the amount of sharing desired in the problem. The diversity of the population is heavily dependent on this sharing parameter and it is often unclear what value it should take. NSGA-II defines a "crowding

distance" $\left(i_{\text {distance }}\right)$ measure which computes the average distance of two points on either side of the individual in question along each objective. The selection process takes into account the number of individuals that each member dominates (referred to as nondomination rank, denoted $i_{\text {rank }}$ ) as well as the crowding distance measure. The guiding of the selection process is outlined using the crowded-comparison operator $\left(\prec_{n}\right)$ as follows:

$i \prec_{n} j$ if $\left(i_{\text {rank }}<j_{\text {rank }}\right)$ or $\left(\left(i_{\text {rank }}=j_{\text {rank }}\right)\right.$ and $\left.\left(i_{\text {distance }}>j_{\text {distance }}\right)\right)$ 
Equation 3-12 states that between two solutions with different non-domination ranks, the solution with lower rank is preferred. If the non-domination ranks are equal, the solution belonging to a less crowded area is desired.

NSGA-II has been shown to produce very promising results on standard multiobjective test problems [2] [31], prompting its inclusion as one of the landmarks for evaluating other MOEAs. It was also shown to be superior to SPEA in almost every measurable fashion. This prompted Zitzler, Thiele and Laumanns to develop its own next generation MOEA, SPEA2. 


\section{The Strength Pareto Evolutionary Algorithm 2 (SPEA2)}

\subsection{Overview}

The Strength Pareto Evolutionary Algorithm 2 [2] presents several improvements over its predecessor SPEA. It boasts an improved fitness assignment scheme which distinguishes individuals in a much more effective fashion. A more precisely guided search process is fostered by a nearest neighbor density technique. Finally, a new archive truncation method guarantees the preservation of boundary solutions to ensure that the entire span of the Pareto front remains intact.

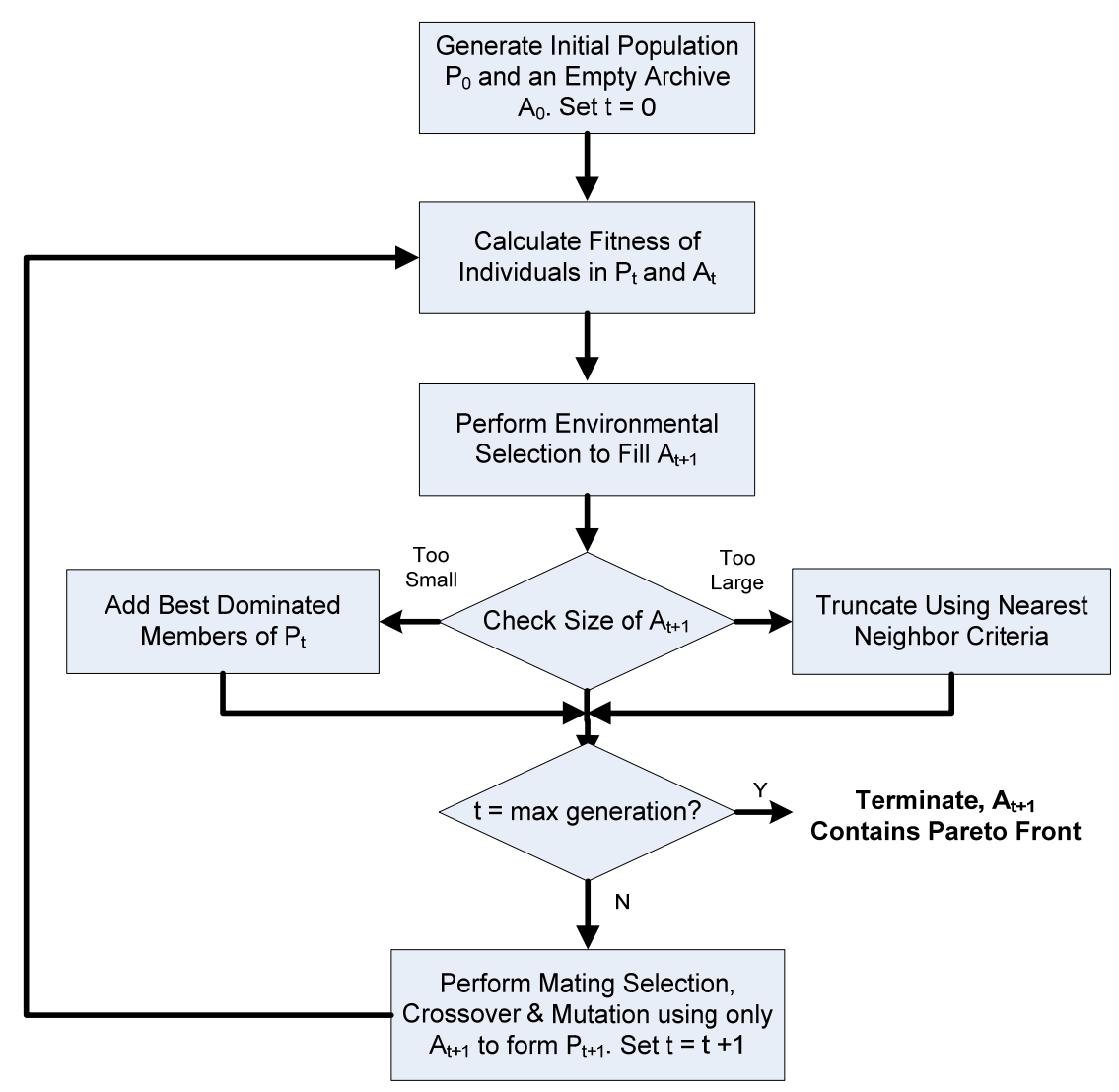

Figure 4-1: SPEA2 Flowchart 
Figure 4-1 depicts the flowchart for SPEA2. An initial population is created by using a random number generator, as was described in Section 3.1.1. An archive of elite solutions, denoted $A_{t}$ where $t$ is the generation number, is utilized in SPEA2 as in SPEA to preserve the best solutions across each generation. However, unlike its predecessor, the archive size in SPEA2 is constant. Also in contrast to SPEA, SPEA2 selects mating pairs only from the archive population. This promotes the reproduction of advantageous characteristics, but at the same time limits the variation in the offspring. Variation can be introduced through the mutation operator. The user decides what type of mating selection, crossover operation, and mutation operator to implement. For the SMRW design problem, tournament selection, uniform crossover, and binary mutation were employed as described in Section 3.1.2.

The details of SPEA2 will be described in the following sections. Section 4.2 will discuss the fitness assignment scheme which includes the nearest-neighbor density technique. Section 4.3 will describe the environmental selection process as well as the techniques used to maintain a constant archive size.

\subsection{Fitness Assignment}

One of the deficiencies that was discovered in SPEA was that individuals who were dominated by the same archive members were assigned identical fitness values. It was irrelevant if one dominated member performed better than the other dominated member in each objective. Both dominated members had equal probability of passing to the next generation in SPEA. This situation forced SPEA to perform more like a random 
search algorithm. Therefore, in SPEA2, each individual's fitness is based upon the solutions that dominate it as well as the solutions that it dominates.

For each individual $i$, a strength value $S(i)$ is determined that represents the number of solutions that it dominates. In mathematical terms,

$$
S(i)=\left|\left\{j \mid j \in P_{t}+A_{t} \wedge i \succ j\right\}\right|
$$

where | | denotes the cardinality of a set and + represents a multi-set union. These strength values are then used to assign a raw fitness, $R(i)$, to each individual.

$$
R(i)=\sum_{j \in P_{t}+A_{t}, j \succ i} S(j)
$$

Thus, the raw fitness of an individual is determined by the strength of its dominators in both the archive and the population [2]. Note that in terms of raw fitness, a low number represents a "good" solution, i.e. it is not dominated by many members. A zero raw fitness would indicate that the particular individual is non-dominated. This idea is illustrated in Figure 4-2 along with a comparison of SPEA’s fitness assignment scheme.
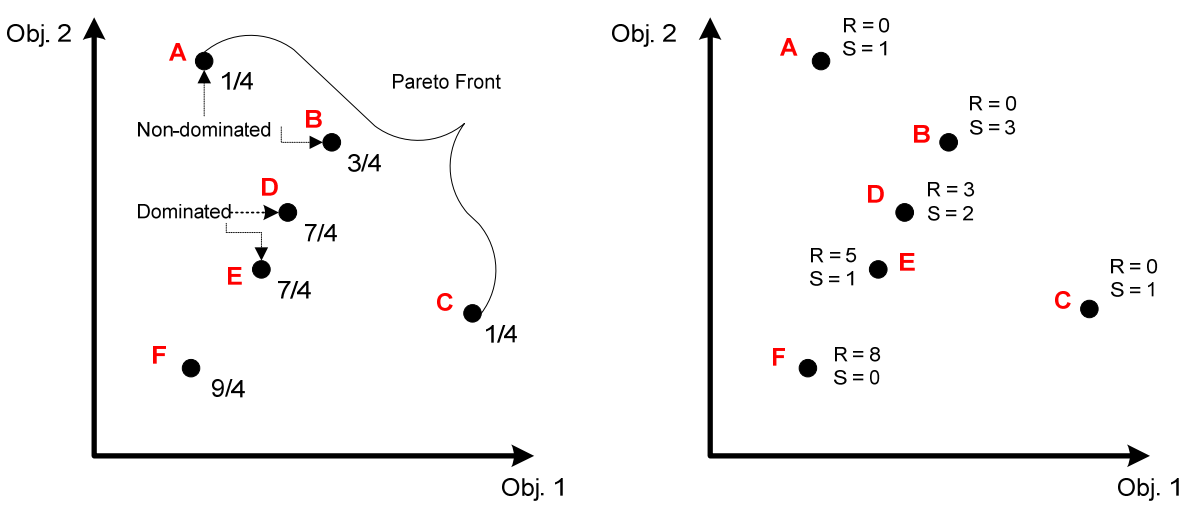

Figure 4-2: Fitness assignment schemes for the same population in $\operatorname{SPEA}^{2}$ (left) and SPEA2 (right) [2].

\footnotetext{
${ }^{2}$ Note that the Pareto front, non-dominated members and dominated members are explicitly indicated in the SPEA diagram for clarity but are also applicable in the SPEA2 diagram.
} 
It is imperative to understand the difference between fitness assignment strategies between SPEA and SPEA2. In Figure 4-2, the letters are used to distinguish between population members for the purpose of this discussion. SPEA's fitness assignment begins by determining the number of solutions that each archive member dominates. In the case depicted by Figure 4-2, there are three non-dominated members (A,B,C) which make up the external archive population and three population members (D,E,F). Members A and C dominate one member: $\mathrm{F}$, while member $\mathrm{B}$ dominates three members: $\mathrm{D}, \mathrm{E}$, and $\mathrm{F}$. These domination counts are then divided the number of population members (3) plus one to produce a fitness value for the archive members. The population members are then assigned a fitness value equal to the summed fitness values of its dominators plus one. Members $\mathrm{D}$ and $\mathrm{E}$ are dominated by $\mathrm{B}$, therefore their fitness values are both equal to member B's fitness plus one - i.e. $3 / 4+1=7 / 4$.

The SPEA2 raw fitness assignment scheme utilizes a strength value (S) and a raw fitness value (R). The strengths of each member are equal to the number of individuals in the population that they dominate and the raw fitness values are equal to the sum of the strengths of each member's dominators. The non-dominated members (A,B,C) have a raw fitness value equal to 0 because they are not dominated by any other member. Members $\mathrm{A}$ and $\mathrm{C}$ dominate one member, $\mathrm{F}$, so their strength is equal to 1 . Member $\mathrm{B}$ dominates 3 members (D,E,F) so its strength value is 3 . Member $\mathrm{D}$ is only dominated by member $\mathrm{B}$, so it is assigned a raw fitness value of 3 . It dominates members $\mathrm{E}$ and $\mathrm{F}$, so it is assigned a strength value of 2 . This assignment process continues for each member of the population. 
Figure 4-2 explicitly shows the deficiency of the SPEA fitness assignment scheme. The two individuals that are dominated by the same member of the archive population ( $\mathrm{D}$ and $\mathrm{E}$ ) are assigned the same fitness value (7/4), despite the fact that one individual (D) is clearly superior to the other (E) in objective space. The SPEA2 fitness assignment remedies this issue. Individual D has a lower raw fitness (3) than individual E (5), indicating that it is indeed a better solution.

Raw fitness is only one part of the SPEA2 fitness measure. To differentiate between individuals that may, by chance, have identical raw fitness values, an additional density measure is incorporated. The inverse of the distance to the k-th nearest neighbor is taken to be the density estimate for a given individual. For every member, the distances to all other members is calculated and sorted in ascending order. The distance to the k-th element, denoted $\sigma_{i}{ }^{k}$, is the point of interest for the density measure. Based on the work of Silverman in [33] the SPEA2 authors determine $\mathrm{k}$ as follows:

$$
k=\sqrt{\operatorname{size}(P)+\operatorname{size}(A)}
$$

The density for each individual is then calculated as:

$$
D(i)=\frac{1}{\sigma_{i}^{k}+2}
$$

Two is added in the denominator to ensure that $D(i)$ takes a value less than one. Finally, the fitness of an individual for the SPEA2 algorithm is calculated as:

$$
F(i)=R(i)+D(i)
$$




\subsection{Environmental Selection}

The process by which the archive population is updated and maintained is referred to as environmental selection. For SPEA2, the first step of this process is to copy all nondominated individuals from the current archive and population to the next generation's archive (i.e. $A_{t+1}$ ). Non-dominated individuals are easily distinguished, as they are guaranteed to have a fitness value less than one. This update process is depicted mathematically by:

$$
A_{t+1}=\left\{i \mid i \in P_{t}+A_{t} \wedge F(i)<1\right\}
$$

At this point, the size of the archive is checked against the pre-defined size limit, denoted $N$. If $\operatorname{size}\left(A_{t+1}\right)=N$ then the environmental selection process is complete and mating selection can be commenced. If $\operatorname{size}\left(A_{t+1}\right)<N$ then the archive is filled with the best dominated population members (an easy task given that the members are already sorted in ascending fitness). The most complicated situation occurs when $\operatorname{size}\left(A_{t+1}\right)>N$.

When the size of the archive is larger than $N$, the SPEA2 truncation operator must be utilized. This algorithm iteratively removes solutions from the archive until it meets the size requirement. This is accomplished by choosing the individual which has the minimum distance to another individual at each stage. Ties are broken by the second smallest distance, etc. The goal of this truncation technique is to maintain the spread of the Pareto front while also preserving the boundary solutions. The technique is illustrated for clearer understanding in Figure 4-3 and is mathematically depicted in Equation 4-7. 


$$
\begin{aligned}
i \leq_{d} j: \Leftrightarrow & \forall 0<k<\operatorname{size}\left(A_{t+1}\right): \sigma_{i}{ }^{k}=\sigma_{j}{ }^{k} \vee \\
& \exists 0<k<\operatorname{size}\left(A_{t+1}\right):\left[\left(\forall 0<l<k: \sigma_{i}^{l}=\sigma_{j}^{l}\right) \wedge \sigma_{i}{ }^{k}<\sigma_{j}{ }^{k}\right]
\end{aligned}
$$

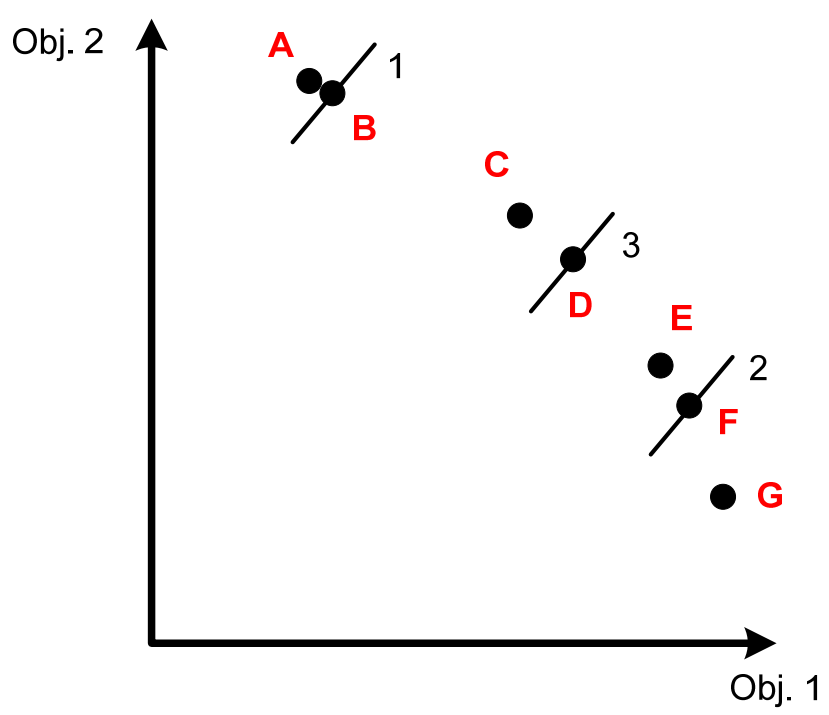

Figure 4-3: Illustration of the SPEA2 archive truncation scheme. $N=4$ is assumed and the numbers on the right side indicate the order of elimination.

As Figure 4-3 depicts, the first solution that is truncated from the Pareto front is the one that is closest to its nearest neighbor (B). Notice that member $\mathrm{A}$ is not removed because it is a boundary solution. Member $\mathrm{G}$ will also not be removed in any truncation scenario. The second elimination is made as a decision between members $\mathrm{E}$ and F. They are the two members with the minimum distance to each other. Member F is selected to be removed because the distance to its next nearest neighbor $(\mathrm{G})$ is smaller than member E's next nearest neighbor (D). The archive size is set to be four, so one more solution must be removed. The next minimum distance between two members is between $\mathrm{C}$ and D. Member D is chosen to be removed because the distance from D-E is smaller than the distance from C-A. The archive is now reduced to its required size of four members. 


\subsection{Verification with the 0/1 Multi-Objective Knapsack Problem}

To verify the functionality of the SPEA2 implementation, the 0/1 multi-objective knapsack problem (MOKP) was utilized [34]. The standard 0/1 single objective knapsack problem is well-known and is frequently used to test optimization algorithms. It is a combinatorial optimization problem in which a set of $n$ items each have an associated profit $(p)$ and weight $(w)$. The goal of the experiment is to choose the items that have the maximum profit while not exceeding the weight capacity $(W)$ of the "knapsack." Mathematically, this is stated as follows:

$$
\begin{aligned}
& \text { maximize } \sum_{j=1}^{n} p_{j} x_{j} \quad \text { subject to } \sum_{j=1}^{n} w_{j} x_{j} \leq W \\
& x_{j} \in\{0,1\} \text { for all } 1 \leq j \leq n
\end{aligned}
$$

To extend this into a multi-objective problem, $m$ knapsacks with different weight capacities are introduced. When an item is selected for inclusion, it is placed in all $m$ knapsacks. The items also have knapsack-specific profits and weights, corresponding to the effects of including an item on each objective. The 0/1 MOKP is defined as follows:

$$
\begin{aligned}
& \text { maximize } \sum_{j=1}^{n} p_{i j} x_{j} \quad \text { subject to } \sum_{j=1}^{n} w_{i j} x_{j} \leq W_{i} \quad 1 \leq i \leq m \\
& x_{j} \in\{0,1\} \text { for all } 1 \leq j \leq n
\end{aligned}
$$

Zitzler and Thiele have developed a comprehensive set of MOKP test data and results $^{3}$ that are referenced in [2], [5] and [29]. For the purposes of verifying the functionality of the SPEA2 implementation, a simple MOKP was chosen for testing. A three knapsack, one-hundred item problem was selected. This problem was robust

\footnotetext{
${ }^{3}$ Available publicly at http://www.tik.ee.ethz.ch/sop/download/supplementary/testProblemSuite/.
} 
enough to exercise SPEA2 but simple enough to accomplish given the computational constraints. A population size of five-hundred members was used along with an archive size of 30, per the experiments in [2]. Although the problem could not be evolved for as many generations as in [2] (50 as opposed to 500), the results were similar to those reported by Zitzler and Thiele. This indicated that the algorithm was indeed functioning as intended and could now be applied to the SMRW design problem. 


\section{SPEA2 Applied to Simultaneous Multi-Mission Radar Waveform Design}

\subsection{Waveform Suite Approach}

Section 1.2 noted the main deficiency of existing multi-mode radar systems: they cannot accomplish multiple missions simultaneously using the same waveform. Section 2.1 highlighted the numerous radar parameters that are of specific interest to accomplishing MTI and SAR missions, some of which are summarized in Table 2-1. This section will aim to define a single waveform, which will be referred to as a waveform suite, that combines different radar parameters to accomplish multiple missions simultaneously.

The RIT Multi-Mission Radar Waveform Tool has been developed in MATLAB ${ }^{\circledR}$ and provides its user the ability to vary a number of different radar parameters. These parameters are:

- $\quad$ Number of pulses per coherent processing interval (CPI)

- $\quad$ Pulse repetition frequency (PRF)

- $\quad$ Center frequency $\left(f_{c}\right)$

- Bandwidth

- Azimuth beam steering angle

- Elevation beam steering angle

For each CPI, the orthogonal waveform suite can be comprised of all or a subset of these parameters. For the purposes of the genetic algorithm, these parameters are binary 
encoded. The specifics of the encoding will be discussed in Section 5.2. Each CPI will contain a binary string that represents the value of each parameter to be used during that particular transmission, which will be known as a sub-waveform. The transmission of a CPI covers a time interval equal to the number of pulses for that CPI times the inverse PRF. The concatenation of the binary encoded sub-waveforms will then make up the waveform suite which will cover the entire mission time. A visual representation of a binary encoded waveform suite with five bit sub-waveforms is depicted in Figure 5-1. In a GA sense, a waveform suite is analogous to an individual or a solution vector. SPEA2 will accept an initial population of waveform suites, evaluate their performance based on the objective functions defined in Section 5.3, and reproduce new waveform suites as described in Chapter 4.

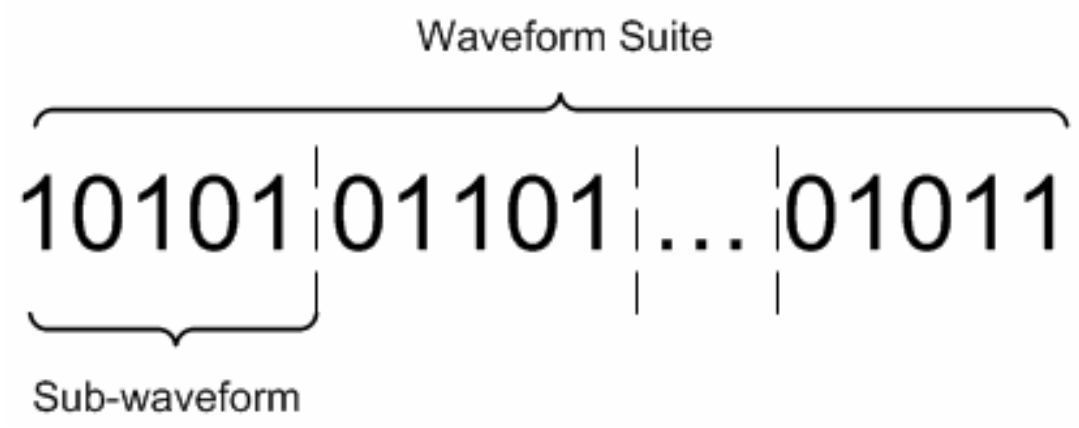

Figure 5-1: Binary encoded waveform suite

\subsection{Parameter Encoding}

The RIT Multi-Mission Radar Waveform Tool allows its user to select the number and type of radar parameters to be varied during each run of the genetic algorithm. A screenshot of the radar parameter selection portion of the RIT MultiMission Radar Waveform Tool graphical used interface (GUI) is shown in Figure 5-2. 
The user first selects the parameter of interest and then defines how many bits should be used to encode its variations. The different values that the parameter is allowed to take are then defined. This process is best described by an example. Consider selecting center frequency as the parameter to be varied in each sub-waveform. The user selects two bits to perform the encoding. This allows the use of four possible values for center frequency. The user defines these center frequency values in an array. The binary encoding would then determine which value should be used in each sub-waveform. For instance, if 00 was selected as the code for the first sub-waveform, the first value in the array would be used for center frequency $(4.92 \mathrm{GHz}$ for the case shown in Figure 5-2). A binary code is generated for each sub-waveform and concatenated with previous sub-waveform codes to comprise a waveform suite, or individual in the GA sense.

\begin{tabular}{|c|c|c|c|c|c|}
\hline No. Pulses: Bits, Values & 2 & \multicolumn{4}{|c|}{$\left[\begin{array}{llll}1 & 8 & 32 & 64\end{array}\right]$} \\
\hline PRF: Bits, Values $(\mathrm{Hz})$ & 1 & \multicolumn{4}{|c|}{$\left[\begin{array}{ll}333 & 500]\end{array}\right.$} \\
\hline (-) Center Freq: Bits, Values $(\mathrm{GHz})$ & 2 & {$[4.92$} & 5.1067 & 5.2933 & $5.48]$ \\
\hline Bandwicth: Bits, Values $(\mathrm{MHz})$ & 1 & \multicolumn{4}{|c|}{$\left[\begin{array}{ll}50 & 50\end{array}\right]$} \\
\hline Az Steer Angle: Values $(\mathrm{MHz})$ & 5 & {$[-60$} & -56.129 & -52.2581 & $-48.387^{\prime}$ \\
\hline El Steer Angle: Values $(\mathrm{MHz})$ & 4 & {$[-4.2$} & -4.2 & -4.2 & 4.2 \\
\hline
\end{tabular}

Figure 5-2: RIT Multi-Mission Radar Waveform Tool: Selection of radar parameters GUI screenshot

If multiple parameters were selected to be varied, their binary codes would be concatenated into a sub-waveform and then combined with other sub-waveforms to define the waveform suite. All non-varied parameters are selected to be the first value in each array and are held constant throughout the experiment. There is no need to encode a constant parameter, as it is used for all sub-waveforms. 


\subsection{Objective Functions}

Four objective functions are utilized to evaluate the success of each waveform suite. These objective functions map the performance of the waveform suite into fitness values that are used by SPEA2 to determine dominated and non-dominated individuals. The success of the SAR mission is measured by estimating the quality of the resulting image. This is accomplished by calculating the peak sidelobe level (PSL) and the integrated sidelobe level (ISL) of the point source spatial response given by Equation 215. The success of an MTI mission is evaluated by a target revisit time specification as well as the number of integrated pulses placed on a target. As described by Section 2.1.1, the number of integrated pulses is directly related to the probability of detection. The following subsections will elaborate on these four objective functions.

\subsubsection{Peak Sidelobe Level \& Integrated Sidelobe Level}

PSL and ISL are discussed together in this subsection because they are inherently related to one another. As Section 2.1.2 noted, a video phase history contains spatial and frequency information for an image. A two-dimensional inverse Fourier transform is then applied to the VPH to create a point spread function (PSF). If the VPH was of infinite size and completely filled, its PSF would represent an ideal point source - i.e. an impulse with all its energy focused in the "main beam.” As the size of the VPH decreases and as it becomes less filled, the power in the PSF spreads out and sidelobes become more pronounced. The result of this effect is that the corresponding output image will be blurred. Reducing the peak sidelobe level and the integrated sidelobe level will thus 
increase the image quality. PSL is defined as the highest power in the normalized PSF that is not part of the main lobe. ISL is summation of all the power in the normalized PSF minus the power in the main lobe. PSL and ISL have been used to measure the imaging quality of array imaging systems in [35] [36], and specifically for SAR imaging systems in [37] [38] [39]. Building upon these works, the following PSL and ISL objective functions, shown in Figure 5-3 and Figure 5-4 respectively, were developed to map SAR mission performance (image quality) to fitness values.

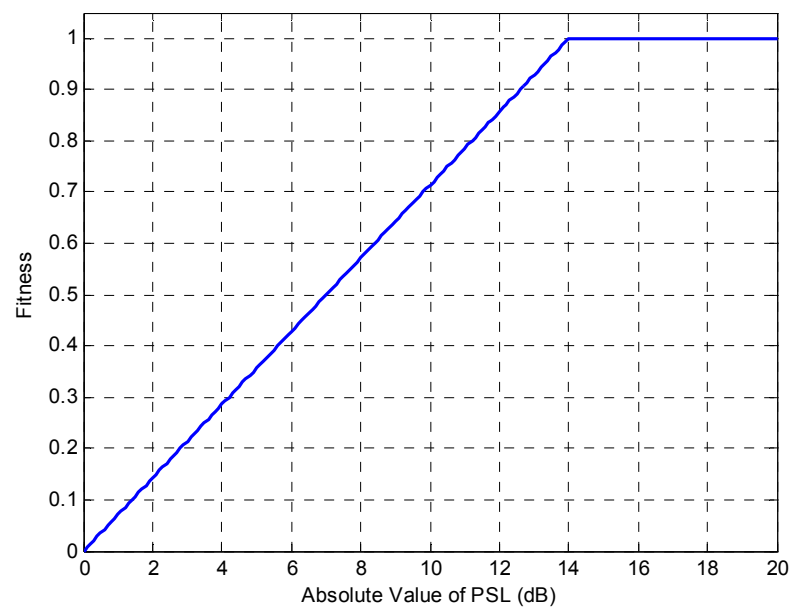

Figure 5-3: PSL SAR objective function. Absolute PSL values greater than $14 \mathrm{~dB}$ result in fitness values of 1 .

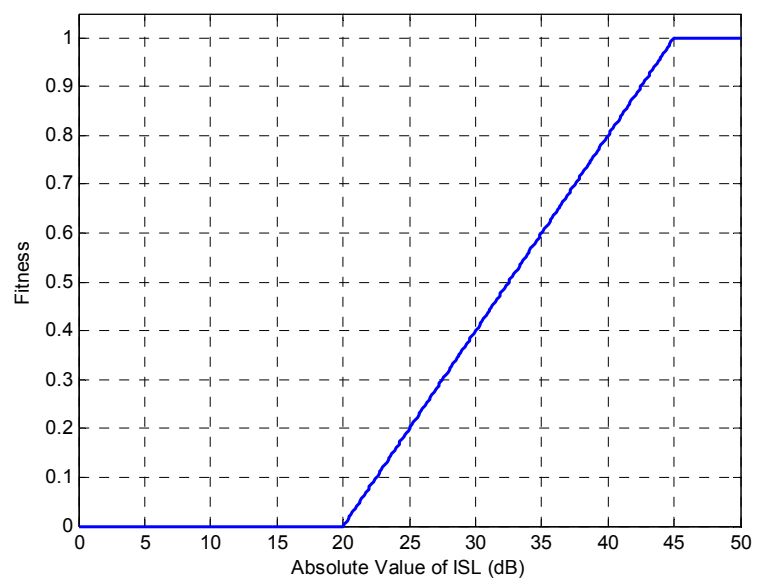

Figure 5-4: ISL SAR objective function. Absolute ISL values greater than $45 \mathrm{~dB}$ result in fitness values of 1 . Any absolute ISL value less than $20 \mathrm{~dB}$ gives a fitness value of 0 . 


\subsubsection{Number of Integrated Pulses}

Section 2.1.1 documented how the number of pulses integrated over a coherent time interval is directly related to the probability of detection. The more pulses that are emitted upon a target, the more likely it is that the target will be detected. The objective function that was developed for the number of integrated pulses was designed to especially favor a larger number of pulses per CPI. A discrete linear function with a non-linear spacing of the independent variable accomplished this task. For all MTI missions, four possible pulse values per CPI were allowed. The discrete objective function to map the number of pulses into fitness is shown in Figure 5-5.

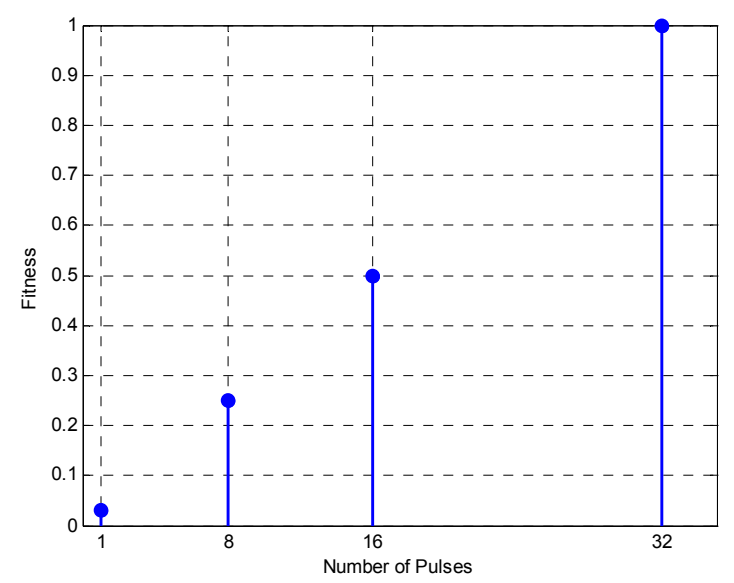

Figure 5-5: Number of pulses MTI objective function. The target number of pulses (the number that gives a fitness value of 1 ) is the maximum allowed in the simulation scenario, 32.

\subsubsection{Revisit Time}

The revisit time for a two-dimensional range cell was chosen as the second objective function for an MTI mission. Depending on the type of target being tracked, long or short revisit times may be required. As Table 2-1 notes, an AMTI mission requires revisit times on the order of ten seconds while a GMTI mission requires revisit times around thirty 
seconds [13]. Consider a target revisit time for a particular mission as $T$ seconds. Revisit times greater than $T$ seconds can degrade mission performance by possibly losing a tracked target of interest. However, revisit times significantly less than $T$ seconds can be considered an inefficient use of resources since other missions (SAR in this case) could be performed. The selection of a revisit time target is highly dependent on the mission requirements. For the scaled MTI mission described in Section 6.2, a revisit time of $0.75 \mathrm{~s}$ within a $3 \mathrm{~s}$ mission was chosen. With the previously described tradeoffs between revisit times longer and shorter than the target in mind, the objective function shown in Figure 5-6 was developed.

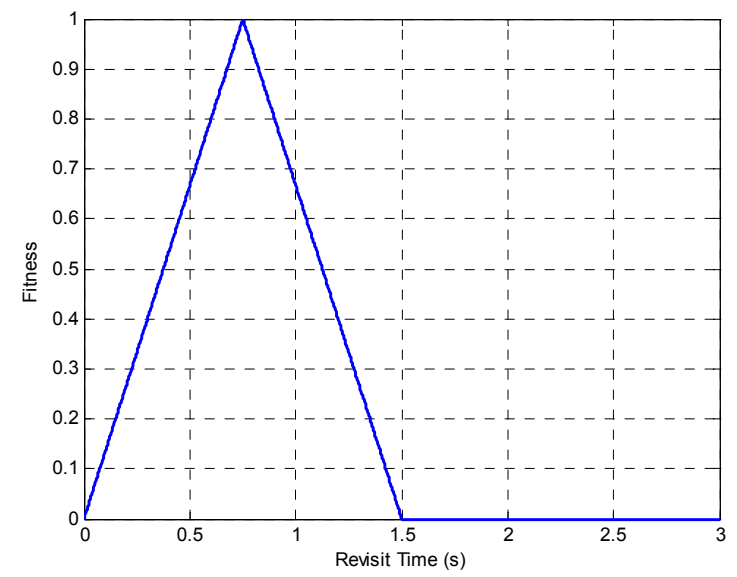

Figure 5-6: Revisit time MTI objective function. The target revisit time is $0.75 \mathrm{~s}$ and results in a fitness value of 1 . The maximum revisit time is $1.5 \mathrm{~s}$ and any value greater than $1.5 \mathrm{~s}$ results in a fitness value of 0 . 


\section{Simulation Scenarios \& Results}

The previous chapters have documented the many constraints placed upon the selection of radar parameters for simultaneous multi-mission radar waveform design. There are also physical limitations of radar systems as well as computational constraints to consider when designing a simulation scenario. Some of the physical limitations include a common PRF and number of emitted pulses for each radar sub-aperture for a given CPI. Also, orthogonal signal separation must occur among simultaneous subaperture transmissions [1]. The main computational constraint to consider is the inverse FFT (IFFT) operation required for the SAR mission. A 2-D IFFT has to be computed for each range cell of the region of interest. This restraint limits both the size of the ROI thus also limiting the length of the mission for a simulation.

For the scaled down SAR scenario described in Section 6.1, the minimum simulation time is $0.346 \mathrm{~s}$ and the minimum size ROI contains 10 x $15=150$ range cells. For a population size of 100 members, each generation of SPEA2 runs for approximately 10 minutes on a $3.2 \mathrm{GHz}, 1 \mathrm{~GB}$ RAM PC. MTI missions require a relatively long simulation run in order to measure criteria such as revisit time, which also requires a larger ROI. To create a scaled physical scenario that can simulate a meaningful MTI mission with a revisit time measure, a simulation time of at least a few seconds must be used. This means that there are more pulse positions during the simulation, which increases the cross-range dimension of the VPH. Thus, a larger IFFT would have to be employed over more cells, because the ROI would increase as well. Since the scaled SAR 
scenario with a simulation time of only $0.346 \mathrm{~s}$ takes approximately 10 minutes per generation to run, it was not possible to integrate a SAR and MTI mission on a single PC. Therefore, it was decided to create two scaled scenarios where each mission could be tested with multiple objectives separately. A procedure is outlined to combine the two scenarios to create a true simultaneous multi-mission simulation that can be performed on a multiple-node parallel processing computer in Section 7.2.

\subsection{Scaled Scenario 1: SAR Mission}

The first simulation scenario was generated to accomplish a SAR mission using peak sidelobe level and integrated sidelobe level as objectives. As noted in Section 5.3.1, PSL and ISL represent a measure of the quality of the SAR image. The scaled physical scenario is depicted in Figure 6-1.

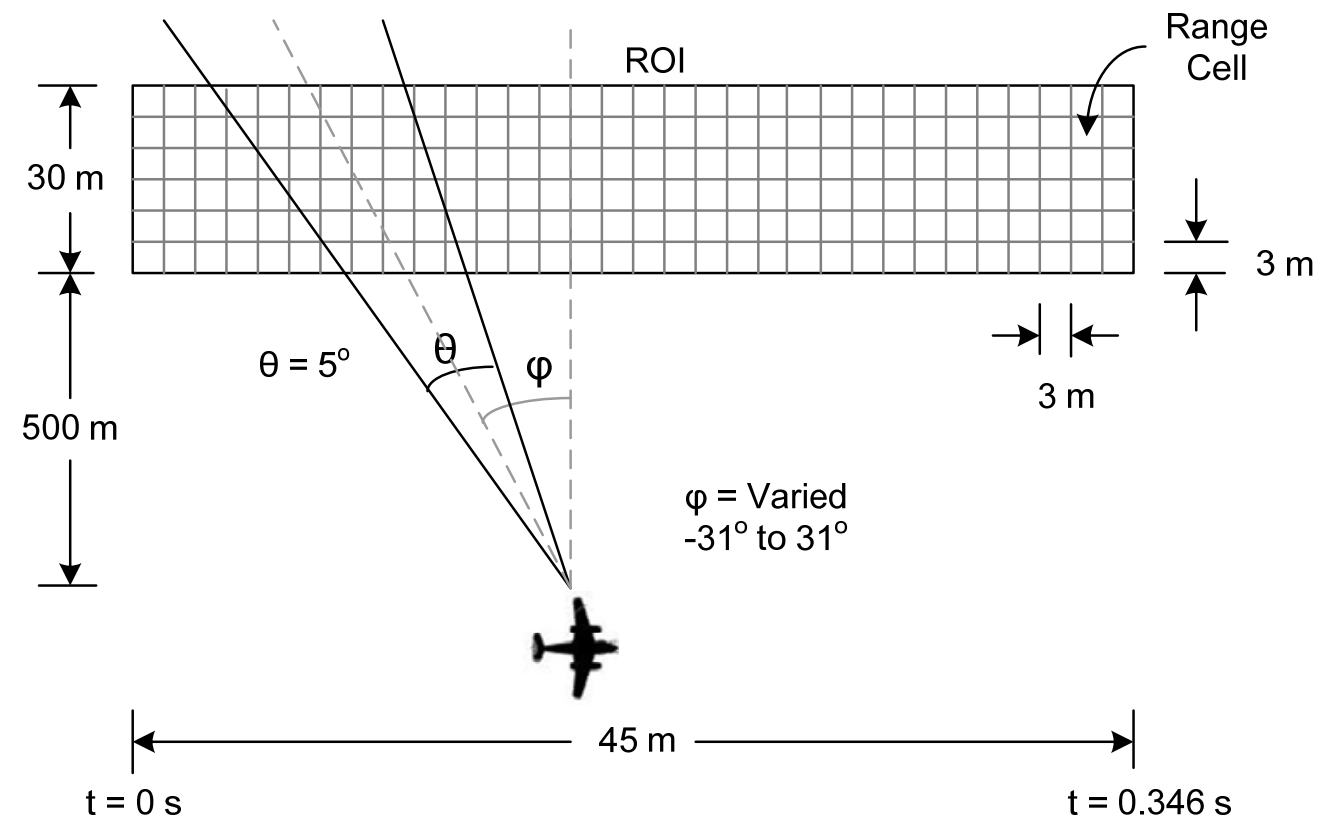

Figure 6-1: Scenario 1 - SAR mission (not drawn to scale) 
While obviously not drawn to scale, Figure 6-1 provides an effective visual representation of the simulated scenario. A typical beamwidth of $\theta=5^{\circ}$ was chosen first and then the length of the ROI $(l)$ was considered. Flying for one SAR effective aperture length $\left(L_{\text {eff }}\right)$ is sufficient for a scaled scenario, so therefore the distance to the ROI $(d)$ determines the length of the ROI by the following simple trigonometric equation:

$$
l=2 d * \tan \left(\frac{\theta}{2}\right)
$$

As documented earlier, it was important to keep the size of the ROI minimal since an IFFT operation must be performed for every range cell. With that restriction in mind, a scaleable distance of $500 \mathrm{~m}$ was selected as $d$. This yielded an $L_{\text {eff }}$ of $45 \mathrm{~m}$, so $l$ was also made to be $45 \mathrm{~m}$. A reasonable choice of $30 \mathrm{~m}$ for the down-range dimension of the rectangular ROI was then made.

The final physical parameters to determine were the dimensions of the range cells which are directly related to the resolution of the SAR system. A resolution of $3 \mathrm{~m}$ in each range dimension was deemed appropriate as it would produce an integer number of computationally manageable cells within the ROI. As noted in Equation 2-6, the crossrange resolution is strictly a function of the actual radar aperture size. The aperture size was chosen to be $6 \mathrm{~m}$ to produce a $3 \mathrm{~m}$ cross-range resolution. Equation 2-7 gives the down-range resolution of the SAR imaging system as a function of the bandwidth of the system. A bandwidth of $50 \mathrm{MHz}$ was chosen to produce a $3 \mathrm{~m}$ down-range resolution.

For this scenario, the aircraft was "flown" from $0 \mathrm{~m}$ to $45 \mathrm{~m}$ at a speed of $130 \mathrm{~m} / \mathrm{s}$. This required a simulation run time of $0.346 \mathrm{~s}$. It was determined that for the 
number of range cells in the ROI, a maximum IFFT size of 256 x 256 could be performed given the computational and time constraints. Therefore, a PRF of $369 \mathrm{~Hz}$ was chosen to produce 128 pulses over the entire simulation run time. This number is important because it is the horizontal dimension of the video phase history. It was chosen to be half the IFFT size in order to improve the resolution of the IFFT for as accurate PSL and ISL readings as possible.

\subsubsection{Full Bandwidth, Single Aperture Experiment}

The first experiment that was performed using this scenario allowed the azimuth pointing angle of a single radar aperture to be varied between -31 to 31 degrees in 2 degree increments. This required the use of five bits per CPI for the binary encoding. Only one pulse per CPI was allowed. This resulted in waveform suites that were 128 x 5 = 640 bits long. A visual representation of a waveform suite for this experiment can be seen in Figure 6-2.

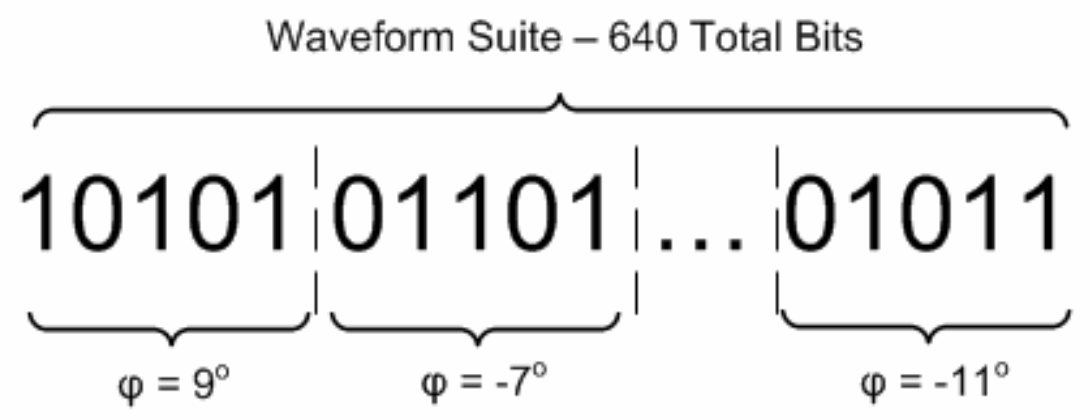

Figure 6-2: Example waveform suite for the full bandwidth, single aperture experiment

As the radar traversed, the VPH for each range cell in the ROI was filled. If a cell was illuminated during a CPI, its VPH was filled with an entire column of 1's for that pulse position since the entire bandwidth was being used. After the radar had flied its 
course, the IFFT operation was performed on each range cell and a PSL and ISL value was determined for each cell. The objective functions shown in Figure 5-3 and Figure 5-4 were then applied to each cell to determine a PSL and ISL fitness value. These fitness values were then averaged over the entire ROI to determine a single PSL and ISL fitness for a waveform suite, or individual.

The full bandwidth, single aperture experiment was performed using different population and archive sizes in SPEA2. Four different simulations were run that alternated population sizes of 50 \& 100 members and archive sizes of 10 \& 20. A summary of the genetic and radar parameters used in the full bandwidth, single aperture experiment are presented in Table 6-1.

\begin{tabular}{|l|c|}
\hline Simulation Parameter & Value \\
\hline No. of sub-apertures & 1 \\
\hline Physical apterture size & $6 \mathrm{~m}$ \\
\hline Effective aperture length & $45 \mathrm{~m}$ \\
\hline Center frequency & $600 \mathrm{MHz}$ \\
\hline Bandwidth & $50 \mathrm{MHz}$ \\
\hline PRF & $369 \mathrm{~Hz}$ \\
\hline No. of pulses & $128 \times 128$ \\
\hline VPH dimensions & $256 \times 256$ \\
\hline IFFT size & $3 \mathrm{~m}$ \\
\hline Cross range resolution & $3 \mathrm{~m}$ \\
\hline Down range resolution & $5^{\circ}$ \\
\hline Beamwidth & $130 \mathrm{~m} / \mathrm{s}$ \\
\hline Airplane velocity & $45 \mathrm{~m}$ \\
\hline ROI cross range size & $30 \mathrm{~m}$ \\
\hline ROI down range size & $500 \mathrm{~m}$ \\
\hline Distance to ROI & PSL \& ISL \\
\hline Azimuth angle (varied) & $-31^{\circ}$ to $31^{\circ}$ in ${ }^{\circ}$ steps \\
\hline Objective functions & $0.346 \mathrm{~s}$ \\
\hline Simulation time & 0.5 \\
\hline Crossover probability & 0.05 \\
\hline Mutation probability & 10 or $20 \mathrm{members}$ \\
\hline SPEA2 archive size & 50 or $100 \mathrm{members}$ \\
\hline SPEA2 population size & 500 \\
\hline Generations & \\
\hline
\end{tabular}

Table 6-1: Summary of simulation parameters for the full bandwidth, single aperture experiment 


\subsubsection{Results}

The following figures display the results from simulations run with all four combinations of population and archive size. The fitness values of the initial and final population members are presented. In addition, the initial and final video phase history and point source response (PSR) for one range cell (the middle cell) are depicted. For the point source response, a center slice in the cross-range and down-range dimension was taken to produce a 2-D representation. A summary table of the PSL and ISL values for this middle cell as the population evolved for each simulation is given at the end of this section. 


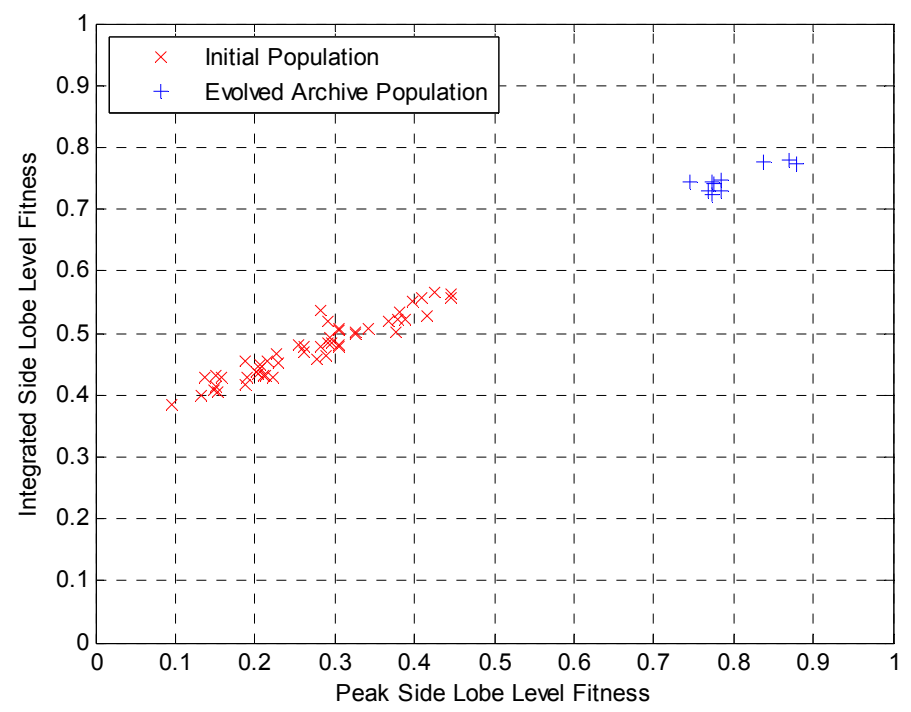

Figure 6-3: Initial population and final archive population for the full bandwidth, single aperture experiment. Population Size $=50$, Archive Size $=10$.
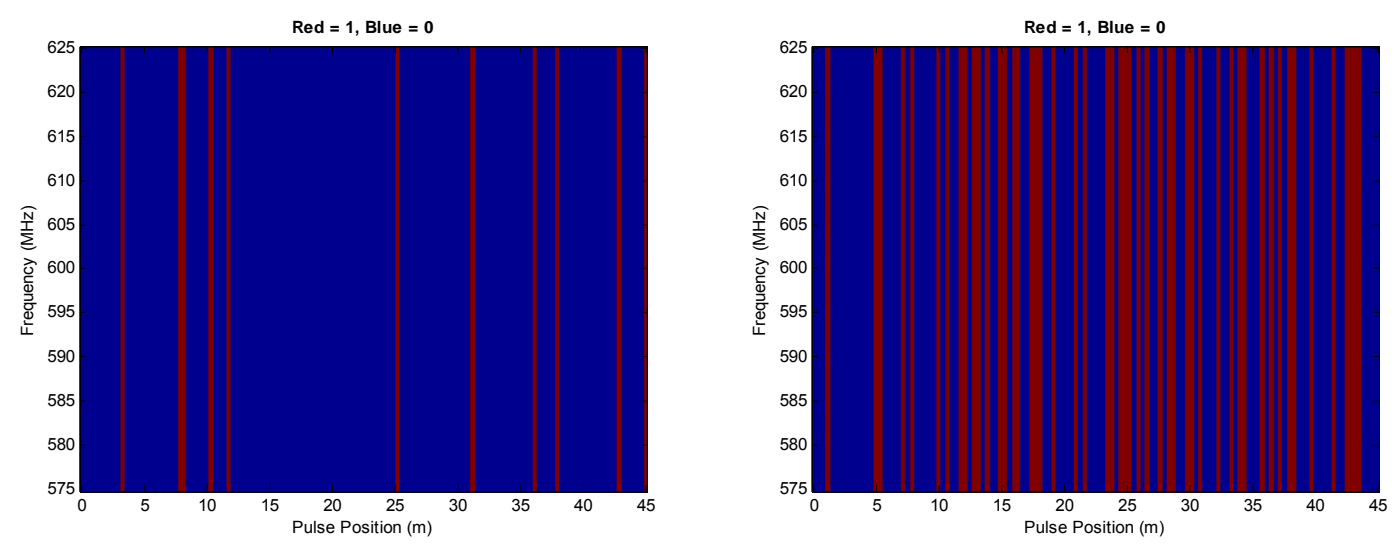

Figure 6-4: Initial (left) and final (right) VPH for the middle cell of the ROI. Population Size $=50$, Archive Size $=10$.
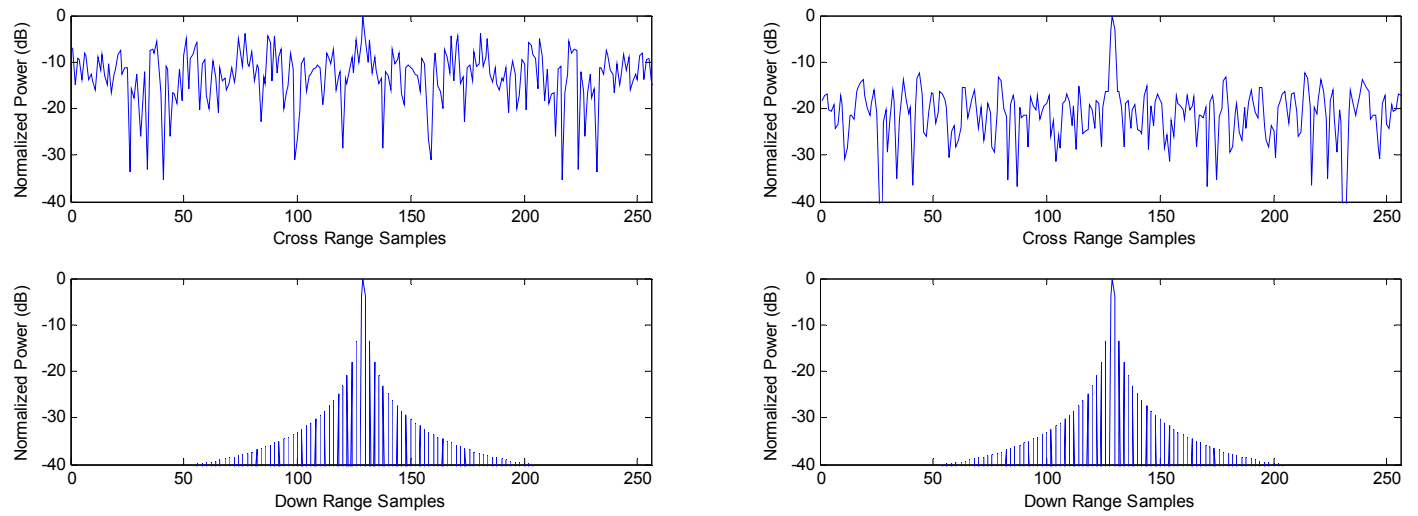

Figure 6-5: Initial (left) and final (right) PSR for the middle cell of the ROI. Population Size $=50$, Archive Size $=10$. 


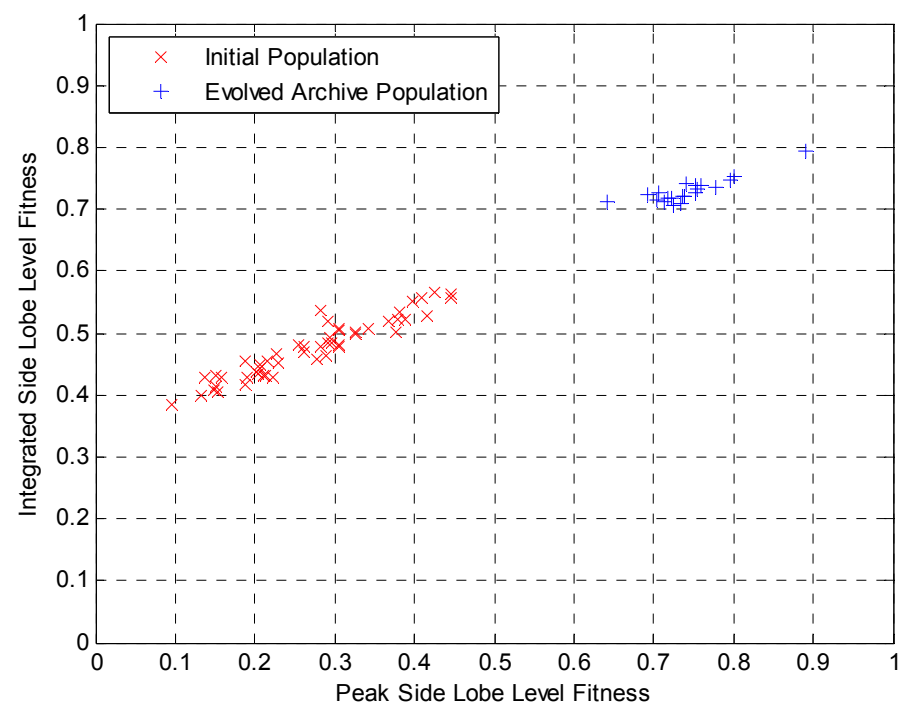

Figure 6-6: Initial population and final archive population for the full bandwidth, single aperture experiment. Population Size $=$ 50, Archive Size $=20$.
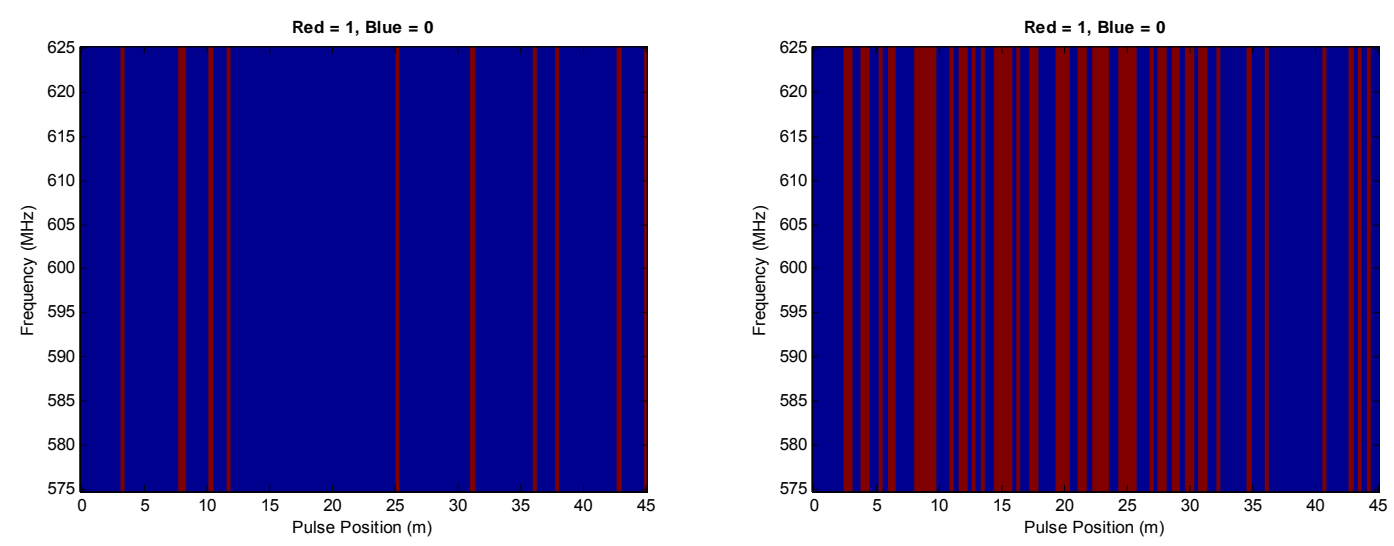

Figure 6-7: Initial (left) and final (right) VPH for the middle cell of the ROI. Population Size $=50$, Archive Size $=20$.
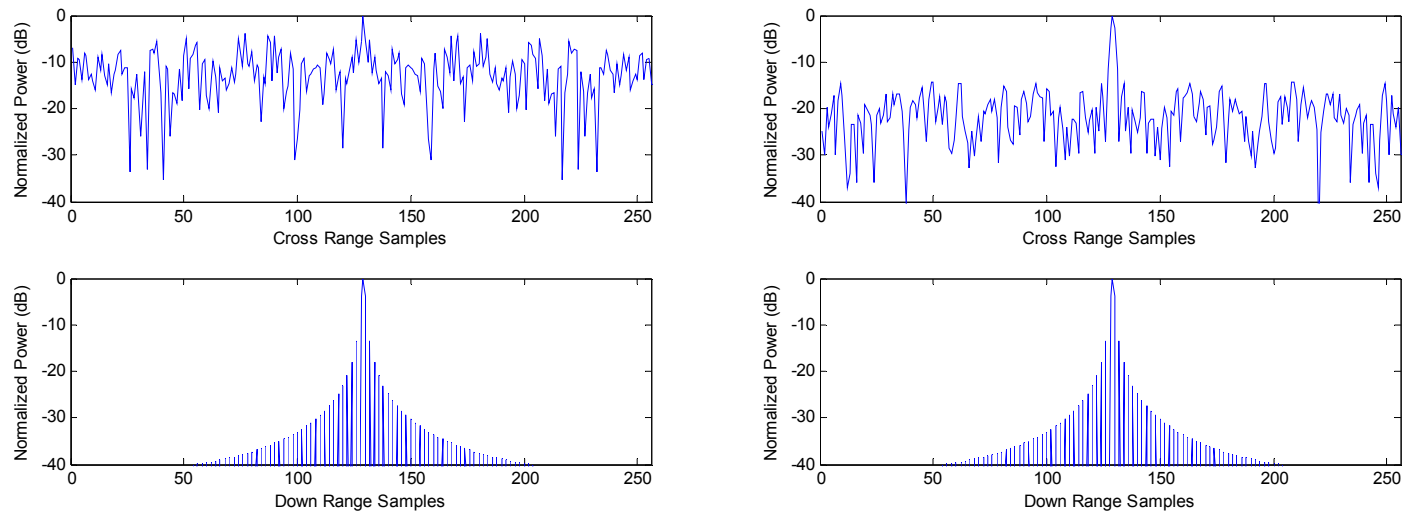

Figure 6-8: Initial (left) and final (right) PSR for the middle cell of the ROI. Population Size $=50$, Archive Size $=20$. 


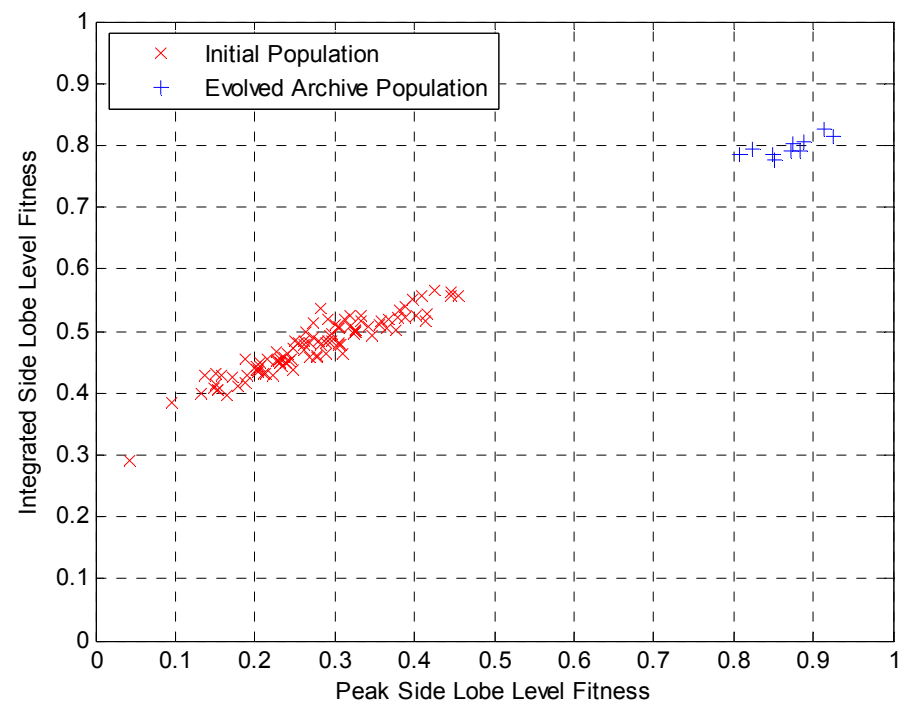

Figure 6-9: Initial population and final archive population for the full bandwidth, single aperture experiment. Population Size $=100$, Archive Size $=10$.
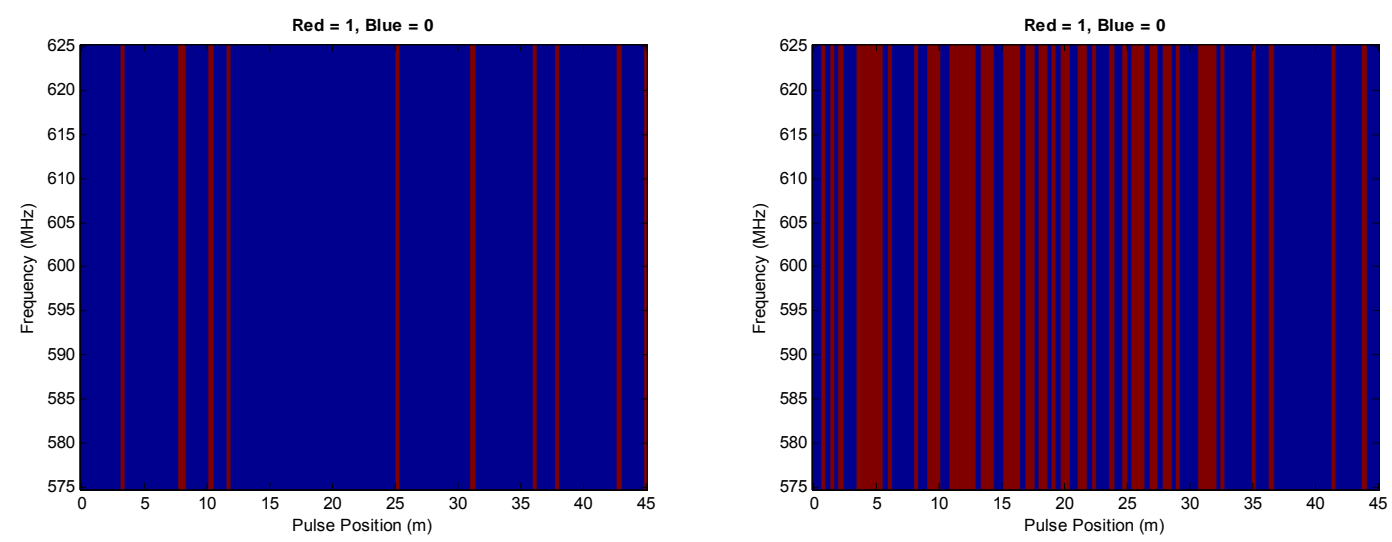

Figure 6-10: Initial (left) and final (right) VPH for the middle cell of the ROI. Population Size $=100$, Archive Size $=10$.
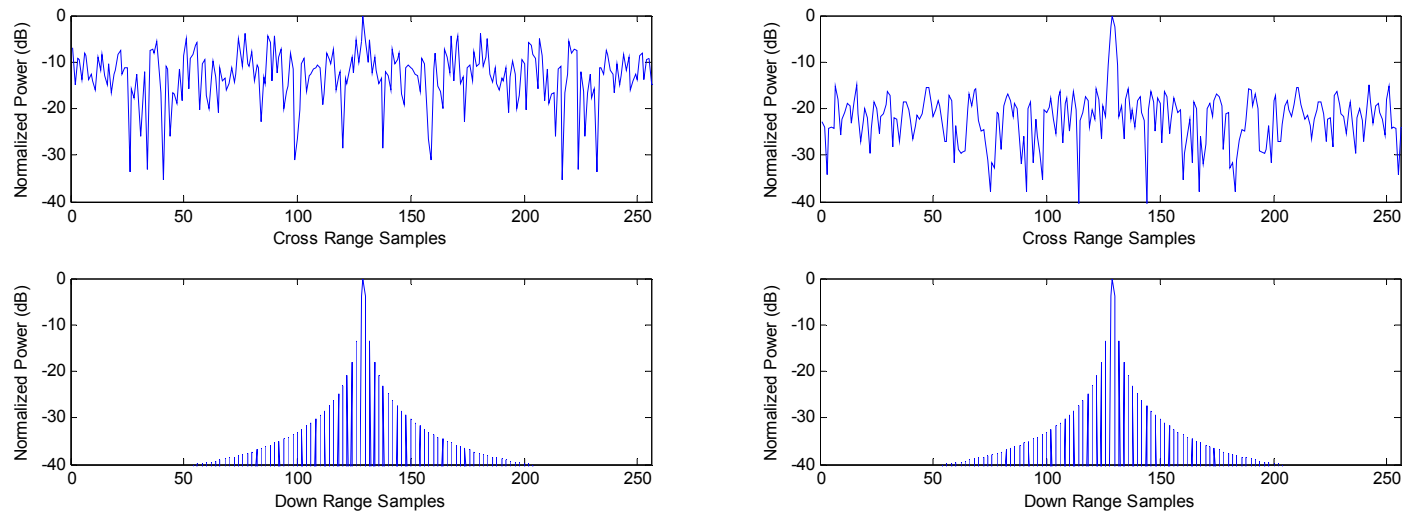

Figure 6-11: Initial (left) and final (right) PSR for the middle cell of the ROI. Population Size $=100$, Archive Size $=10$. 


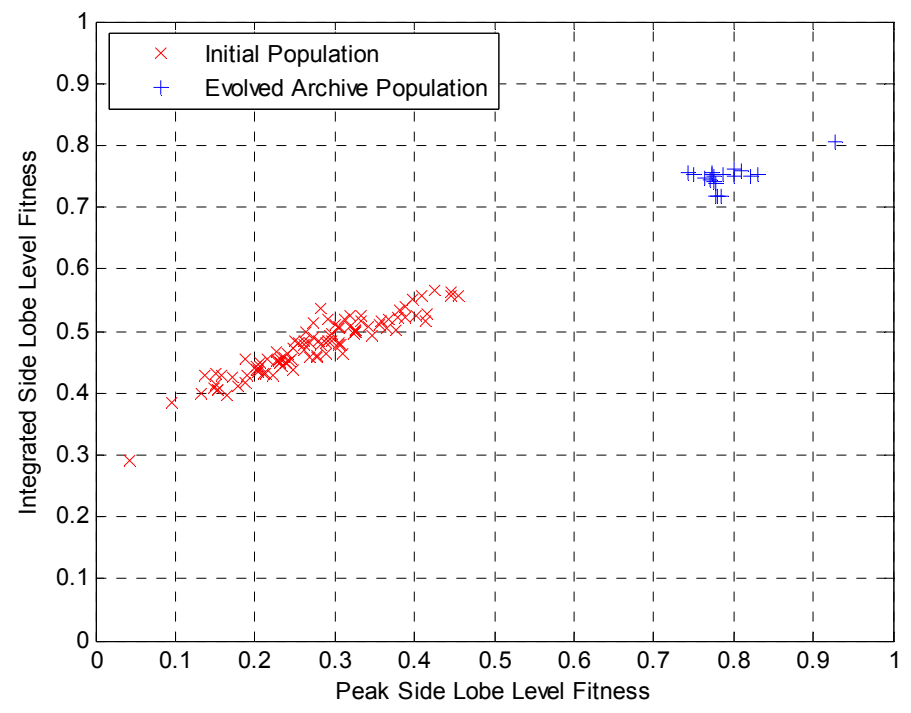

Figure 6-12: Initial population and final archive population for the full bandwidth, single aperture experiment. Population Size $=100$, Archive Size $=20$.
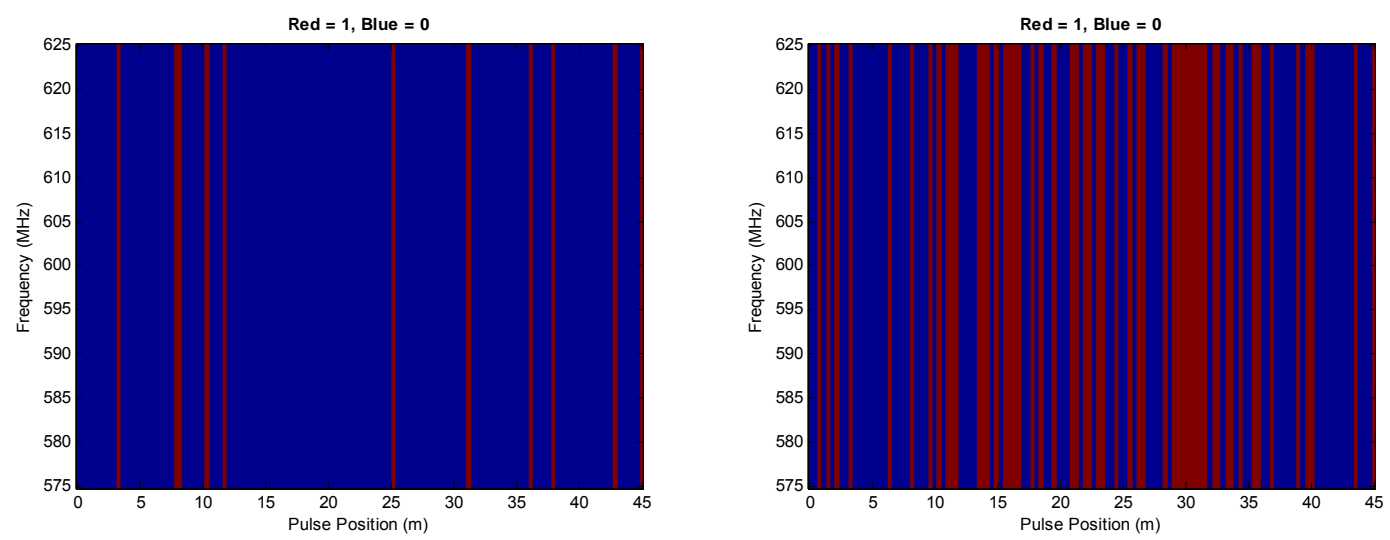

Figure 6-13: Initial (left) and final (right) VPH for the middle cell of the ROI. Population Size $=100$, Archive Size $=20$.
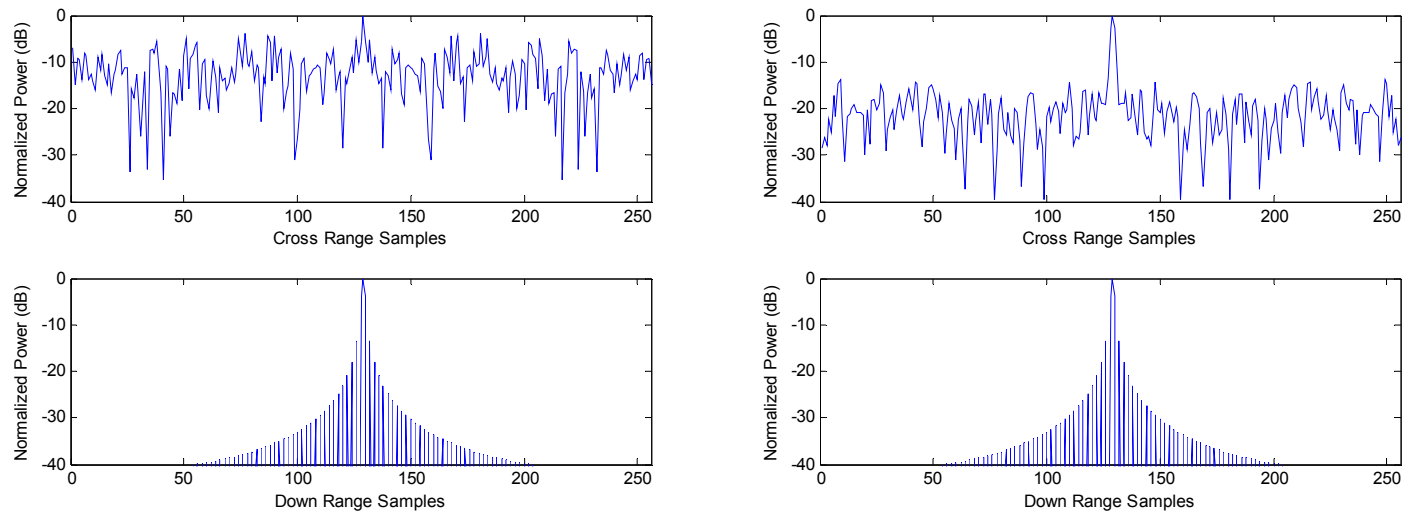

Figure 6-14: Initial (left) and final (right) PSR for the middle cell of the ROI. Population Size $=100$, Archive Size $=20$. 


\begin{tabular}{|c|c|c|c|c|c|c|c|c|c|c|}
\hline \multicolumn{3}{|c|}{ 50 Pop. Members - 10 Arch. Size - Middle Cell of ROI } & \multicolumn{3}{c|}{ 100 Pop. Members - 10 Arch. Size - Middle Cell of ROI } \\
\hline Generation & PSL (dB) & PSL Fitness & ISL (dB) & ISL Fitness & Generation & PSL (dB) & PSL Fitness & ISL (dB) & ISL Fitness \\
\hline 0 (initial) & -3.95 & 0.28 & -31.79 & 0.47 & 0 (initial) & -3.95 & 0.28 & -31.79 & 0.47 \\
\hline 50 & -13.29 & 0.95 & -39.81 & 0.79 & 50 & -11.90 & 0.85 & -39.88 & 0.79 \\
\hline 100 & -12.22 & 0.87 & -39.80 & 0.79 & 100 & -13.46 & 0.96 & -40.54 & 0.82 \\
\hline 200 & -13.46 & 0.96 & -40.43 & 0.82 & 200 & -13.46 & 0.96 & -40.54 & 0.82 \\
\hline 300 & -13.46 & 0.96 & -40.43 & 0.82 & 300 & -13.46 & 0.96 & -40.82 & 0.83 \\
\hline 400 & -12.75 & 0.91 & -39.68 & 0.79 & 400 & -13.46 & 0.96 & -41.22 & 0.85 \\
\hline 500 & -12.43 & 0.89 & -40.03 & 0.80 & 500 & -13.46 & 0.96 & -41.22 & 0.85 \\
\hline 50 Pop. Members - 20 Arch. Size - Middle Cell of ROI & 100 Pop. Members - 20 Arch. Size - Middle Cell of ROI \\
\hline Generation & PSL (dB) & PSL Fitness & ISL (dB) & ISL Fitness & Generation & PSL (dB) & PSL Fitness & ISL (dB) & ISL Fitness \\
\hline 0 (initial) & -3.95 & 0.28 & -31.79 & 0.47 & 0 (initial) & -3.95 & 0.28 & -31.79 & 0.47 \\
\hline 50 & -11.83 & 0.84 & -38.58 & 0.74 & 50 & -12.03 & 0.86 & -39.80 & 0.79 \\
\hline 100 & -11.62 & 0.83 & -38.15 & 0.73 & 100 & -12.80 & 0.91 & -40.18 & 0.81 \\
\hline 200 & -13.37 & 0.95 & -39.73 & 0.80 & 200 & -13.22 & 0.94 & -39.86 & 0.79 \\
\hline 300 & -13.46 & 0.96 & -40.68 & 0.83 & 300 & -13.46 & 0.96 & -39.90 & 0.80 \\
\hline 400 & -13.46 & 0.96 & -40.68 & 0.83 & 400 & -13.46 & 0.96 & -40.68 & 0.83 \\
\hline 500 & -13.46 & 0.96 & -40.68 & 0.83 & 500 & -13.46 & 0.96 & -40.68 & 0.83 \\
\hline
\end{tabular}

Table 6-2: Summary of the evolution of PSL and ISL values for each full bandwidth, single aperture simulation

The 100 population, 10 archive size experiment was repeated using five different initial random seeds. This was done to verify the results that were obtained. The following fitness plot depicts the initial population and the final archive population for the five starting seeds, each represented by a different color. Figure 6-16 shows a closer view of the archive population of Figure 6-15.

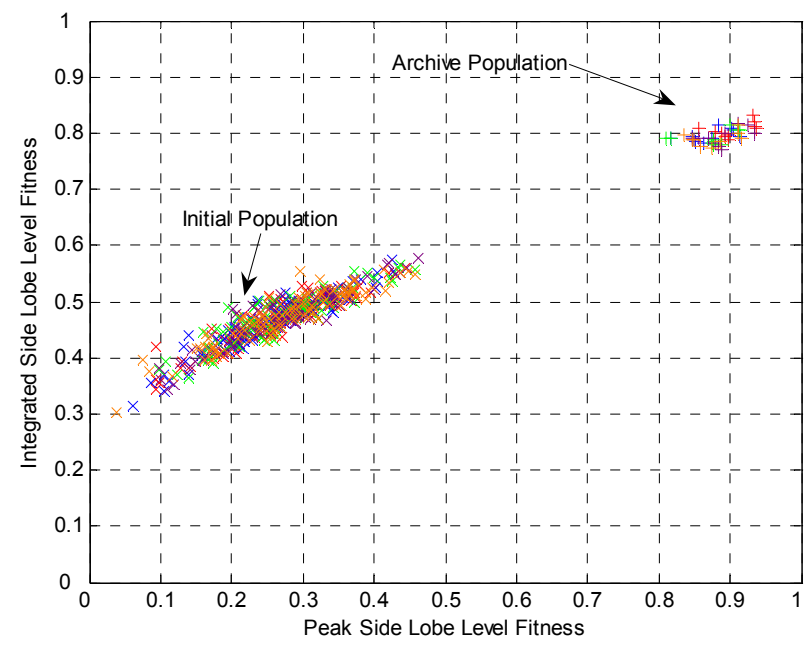

Figure 6-15: Initial population and final archive population using five different random seeds for the full bandwidth, single aperture experiment. Population Size $=100$, Archive Size $=10$. 


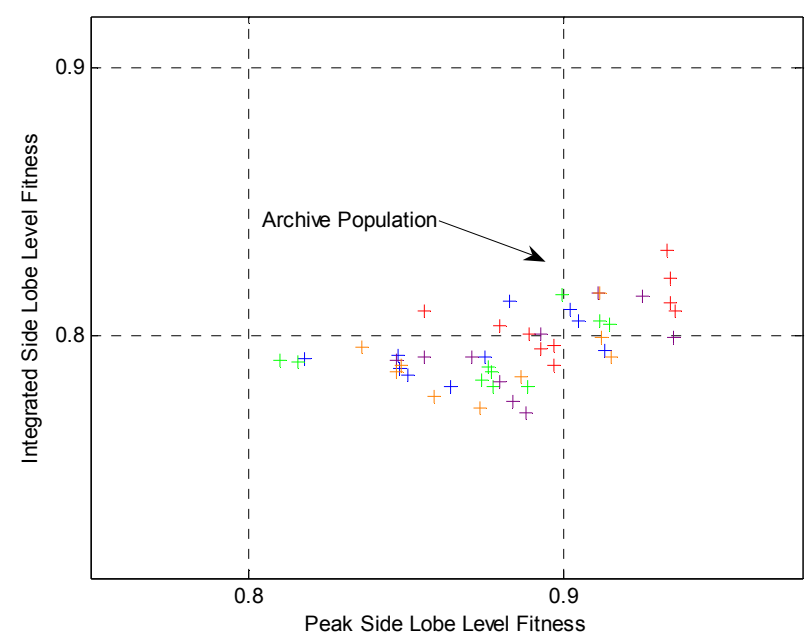

Figure 6-16: Archive population using five different random seeds for the full bandwidth, single aperture experiment. Population Size $=100$, Archive Size $=10$.

\subsubsection{Analysis}

In each simulation, there was a significant increase in both PSL and ISL fitness from the initial population to the final archive population. In the simulations where an archive size of 20 was used (Figure 6-6, Figure 6-12), it can be seen that the algorithm evolved to find one clearly superior, non-dominated archive member. However, the best results in terms of PSL and ISL fitness result from the simulation using 100 population members and 10 archive members shown in Figure 6-9. Two non-dominated archive members with high fitness values in each dimension are reported. Table 6-2 agrees with this assessment, as the PSL fitness and ISL fitness shown for the middle cell are the highest for the 100 population members and 10 archive members simulation. However, it is important to note here that the results reported in Table 6-2 are only for one cell out of the entire ROI. The fitness for this individual cell may actually decrease as the population 
evolves, but the average fitness across the ROI would increase. This phenomenon occurs for several generations as reported in Table 6-2.

In accordance with the increase in population fitness, the VPH and PSR depict a vast improvement from their initial states. In each simulation, the VPH becomes much more filled with energy as the population evolves. In correspondence to this, the normalized power in the cross-range dimension of the PSR drops significantly from its initial to final state. Note that the PSR in the down-range dimension represents a sinc function and does not change from its initial to final state because the system is employing its full bandwidth. When energy hits a range cell in this experiment, the entire down-range spectrum of the VPH is filled. Therefore, the PSR has structure in only the cross-range dimension for this experiment.

Finally, an important result to note is the amount of VPH space that is empty for the evolved archive member (Figure 6-4, Figure 6-7, Figure 6-10, Figure 6-13). The final designed waveform suite produces good PSL and ISL measures and there are still some pulse positions that are empty in the VPH. This indicates that there are instances within the SAR mission where another mission, like MTI, could be accomplished.

\subsubsection{Split Bandwidth, Multiple Sub-Aperture Experiment}

For this experiment, rather than the entire bandwidth being used for the radar signal, it was split into four equal sub-bands. This required the use of four sub-apertures, each radiating at their own center frequency. An azimuth pointing angle had to be encoded for each sub-aperture, as it was allowed to be varied between -31 to 31 degrees in 2 degree increments. This required the use of 20 bits per CPI for the binary encoding. 
Only one pulse per CPI was used. This resulted in waveform suites that were 128 x 5 x 4 $=2560$ bits long. A visual representation of a waveform suite for this experiment can be seen in Figure 6-17.

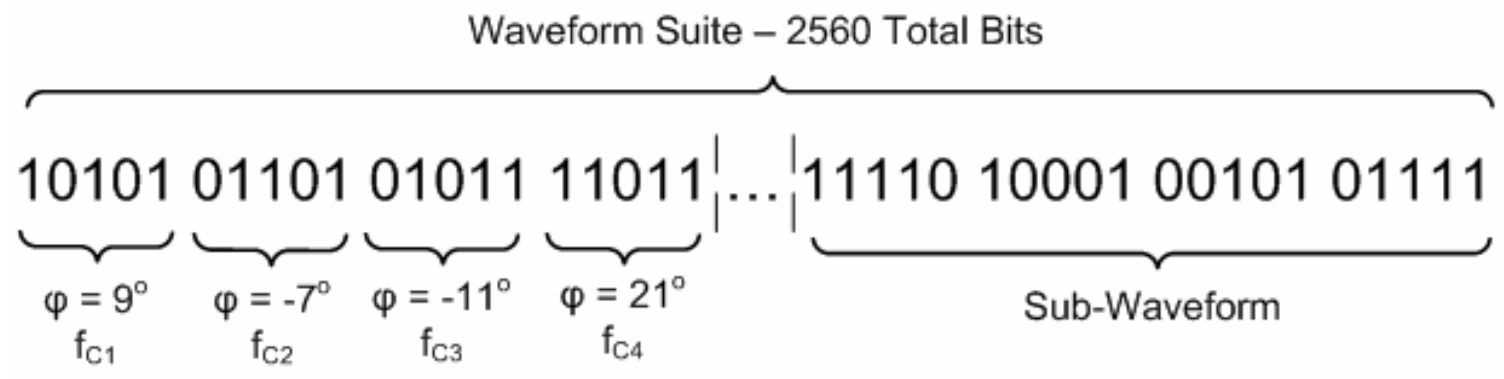

Figure 6-17: Example waveform suite for the split bandwidth, multiple sub-aperture experiment

As the radar traversed, the VPH for each range cell in the ROI was filled. If a cell was illuminated during a CPI by one of the four frequency bands, its VPH was filled with 1's for that frequency band and pulse position. After the radar had flied its course, the IFFT operation was performed on each range cell and a PSL and ISL value was determined for each cell. The objective functions shown in Figure 5-3 and Figure 5-4 were then applied to each cell to determine a PSL and ISL fitness value. These fitness values were then averaged over the entire ROI to determine a single PSL and ISL fitness for a waveform suite, or individual.

The split bandwidth, multiple sub-aperture experiment was performed using different population and archive sizes in SPEA2. Four different simulations were run that alternated population sizes of 50 \& 100 members and archive sizes of $10 \& 20$. A summary of the genetic and radar parameters used in the split bandwidth, multiple subaperture experiment are presented in Table 6-3. 


\begin{tabular}{|l|c|}
\hline Simulation Parameter & Value \\
\hline No. of sub-apertures & 4 \\
\hline Physical apterture size & $6 \mathrm{~m}$ \\
\hline Effective aperture length & $45 \mathrm{~m}$ \\
\hline Center frequencies & $581.25,593.75,606.25,618.75 \mathrm{MHz}$ \\
\hline Bandwidth & $369 \mathrm{Mz}$ \\
\hline PRF & 1 \\
\hline No. of pulses & $128 \times 128$ \\
\hline VPH dimensions & $256 \times 256$ \\
\hline IFFT size & $3 \mathrm{~m}$ \\
\hline Cross range resolution & $3 \mathrm{~m}$ \\
\hline Down range resolution & $5^{\circ}$ \\
\hline Beamwidth & $130 \mathrm{~m} / \mathrm{s}$ \\
\hline Airplane velocity & $45 \mathrm{~m}$ \\
\hline ROI cross range size & $30 \mathrm{~m}$ \\
\hline ROI down range size & $500 \mathrm{~m}$ \\
\hline Distance to ROI & PSL \& ISL \\
\hline Azimuth angle (varied) & $0.346 \mathrm{~s}$ \\
\hline Objective functions & 0.5 \\
\hline Simulation time & 0.05 \\
\hline Crossover probability & 10 or $20 \mathrm{members}$ \\
\hline Mutation probability & 50 or $100 \mathrm{members}$ \\
\hline SPEA2 archive size & 500 \\
\hline SPEA2 population size & to $31^{\circ} \mathrm{in} 2^{\circ}$ steps \\
\hline Generations & \\
\hline
\end{tabular}

Table 6-3: Summary of simulation parameters for the split bandwidth, multiple sub-aperture experiment

\subsubsection{Results}

The following figures display the results from simulations run with all four combinations of population and archive size. The fitness values of the initial and final population members are presented. In addition, the initial and final video phase history and point source response (PSR) for one range cell (the middle cell) are depicted. For the point source response, a center slice in the cross-range and down-range dimension was taken to produce a 2-D representation. A summary table of the PSL and ISL values for this middle cell as the population evolved for each simulation is given at the end of this section. 


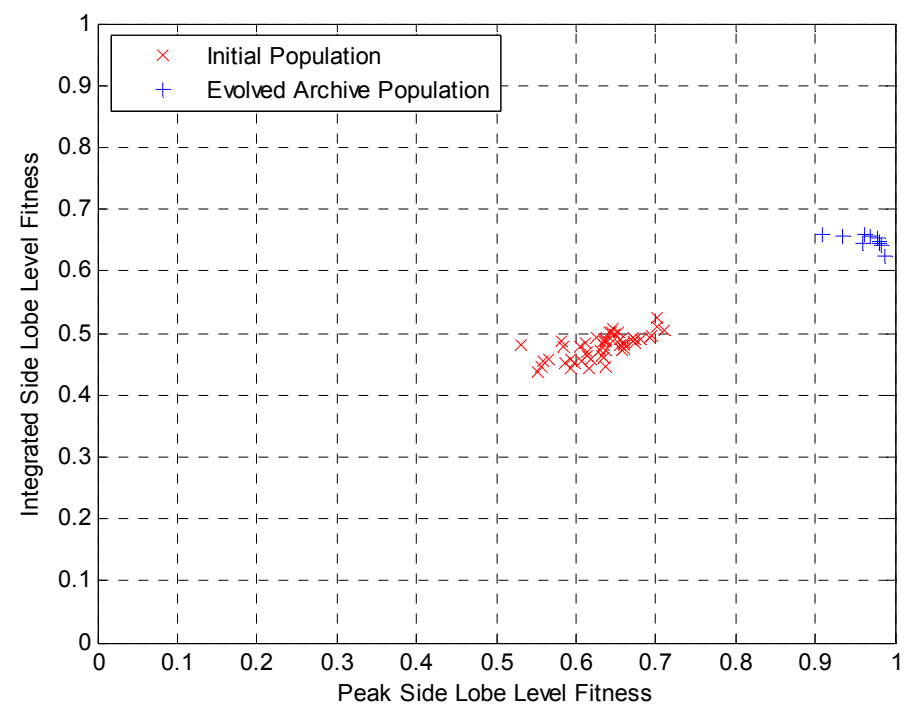

Figure 6-18: Initial population and final archive population for the split bandwidth, multiple subaperture experiment. Population Size $=50$, Archive Size $=10$.
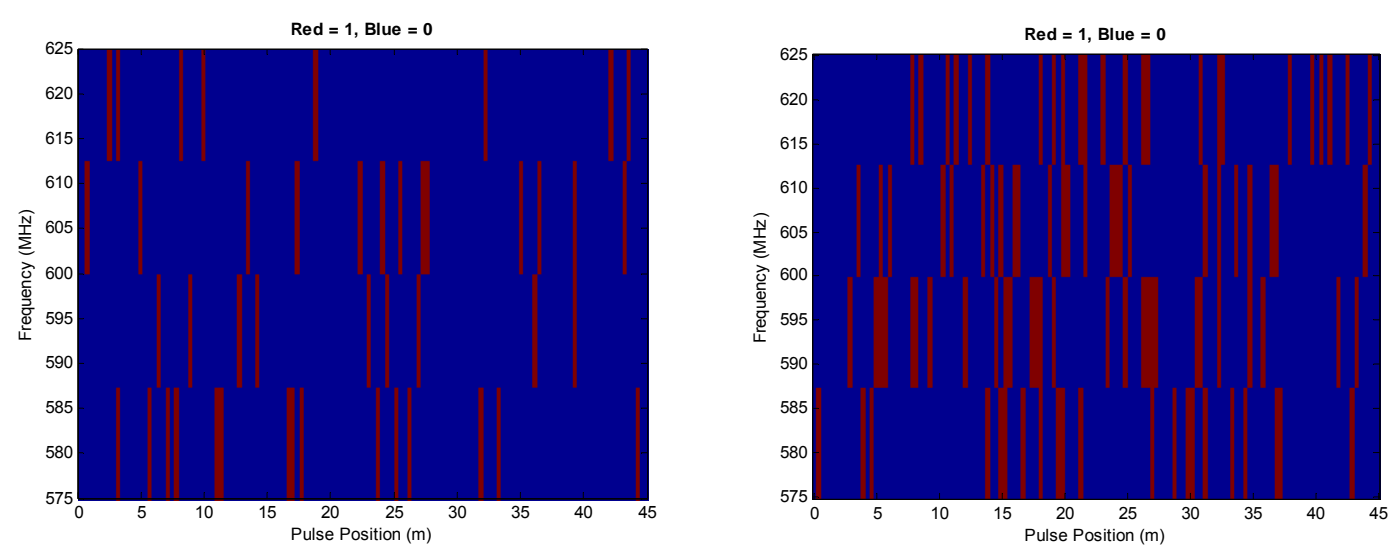

Figure 6-19: Initial (left) and final (right) VPH for the middle cell of the ROI. Population Size $=50$, Archive Size $=10$.
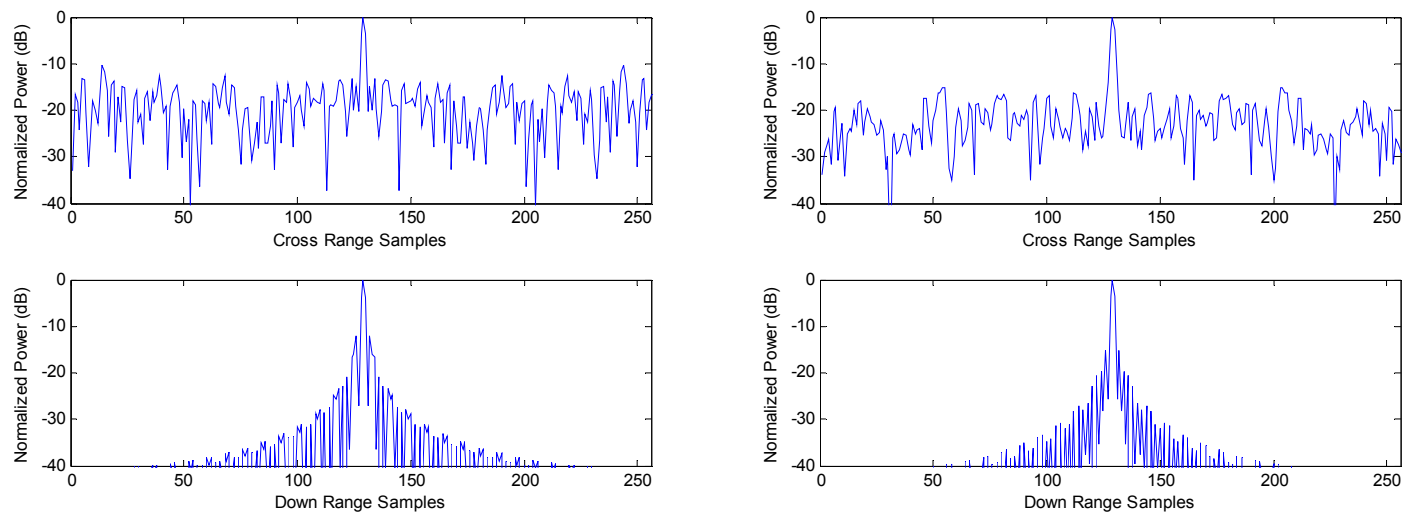

Figure 6-20: Initial (left) and final (right) PSR for the middle cell of the ROI. Population Size $=50$, Archive Size $=10$. 


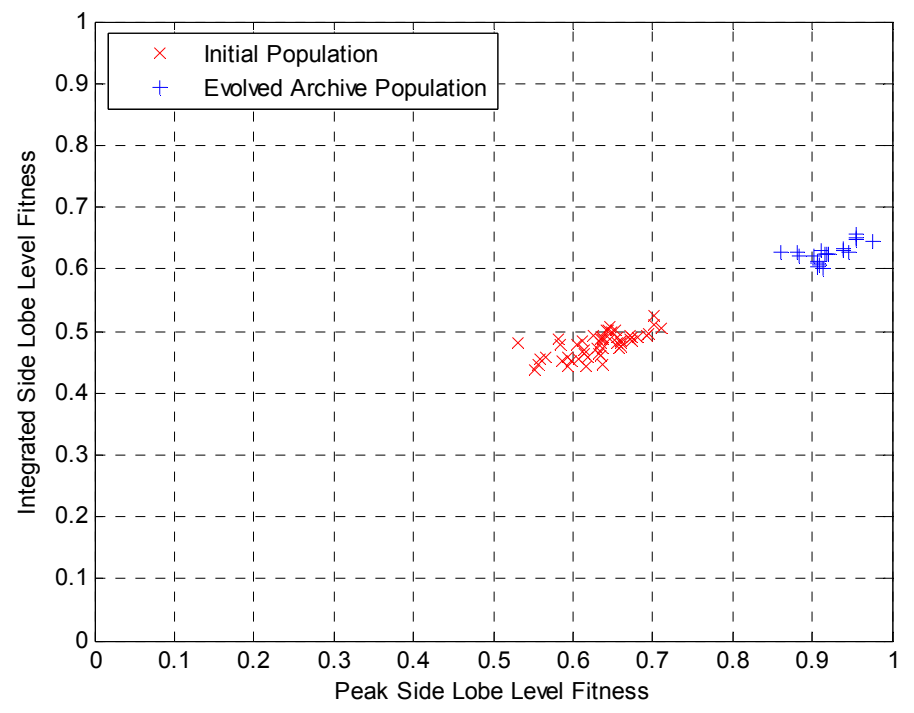

Figure 6-21: Initial population and final archive population for the split bandwidth, multiple subaperture experiment. Population Size $=50$, Archive Size $=20$.
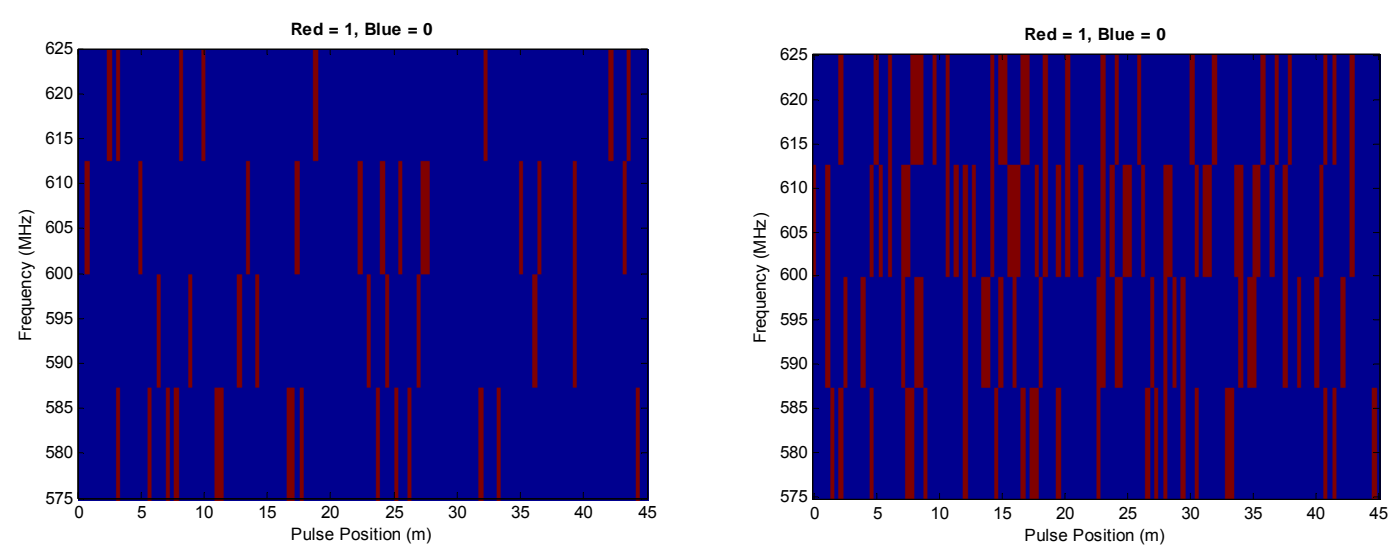

Figure 6-22: Initial (left) and final (right) VPH for the middle cell of the ROI. Population Size $=50$, Archive Size $=20$.
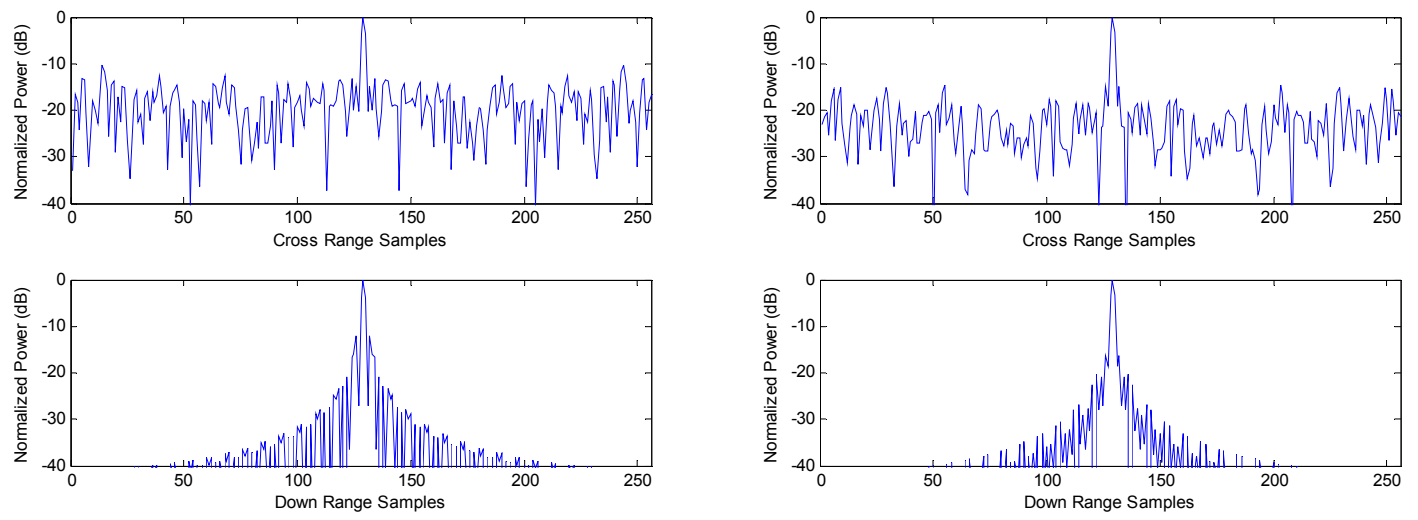

Figure 6-23: Initial (left) and final (right) PSR for the middle cell of the ROI. Population Size $=50$, Archive Size $=20$. 


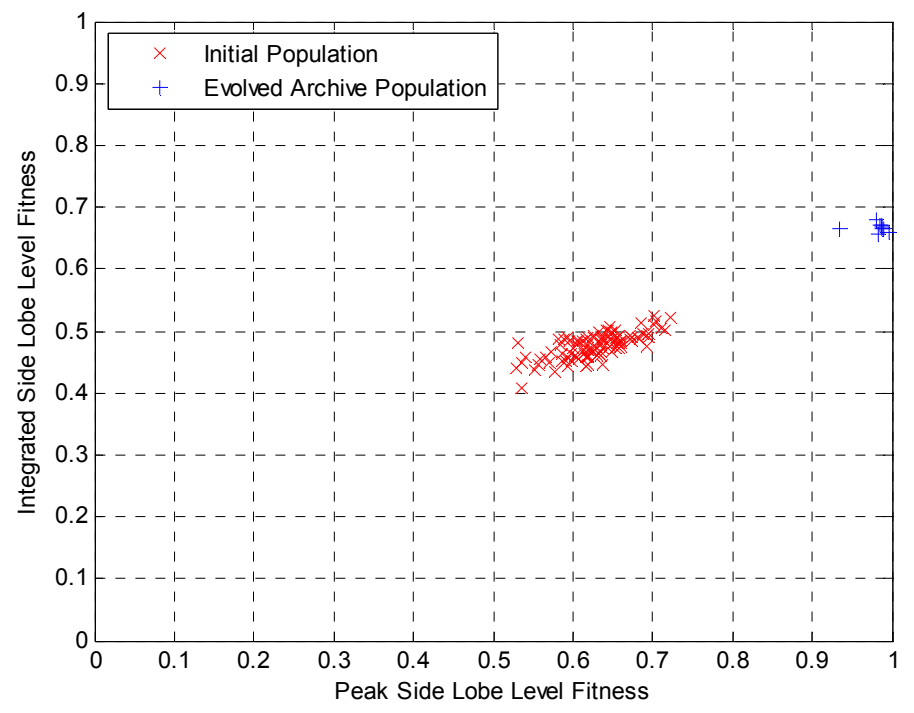

Figure 6-24: Initial population and final archive population for the split bandwidth, multiple subaperture experiment. Population Size $=100$, Archive Size $=10$.
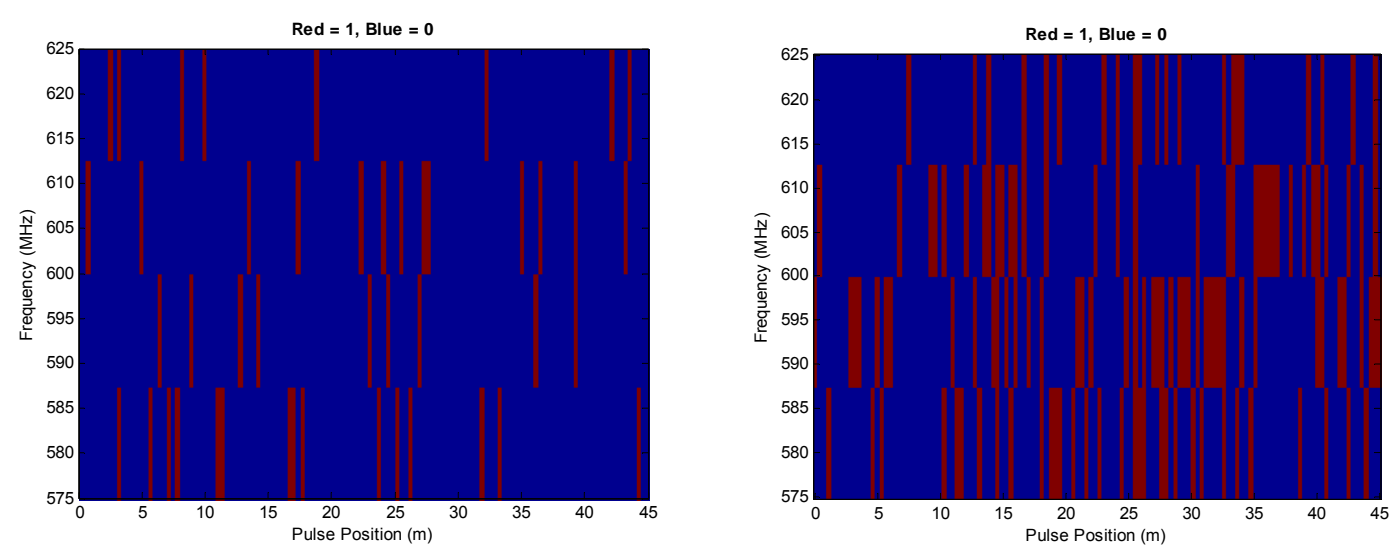

Figure 6-25: Initial (left) and final (right) VPH for the middle cell of the ROI. Population Size $=100$, Archive Size $=10$.
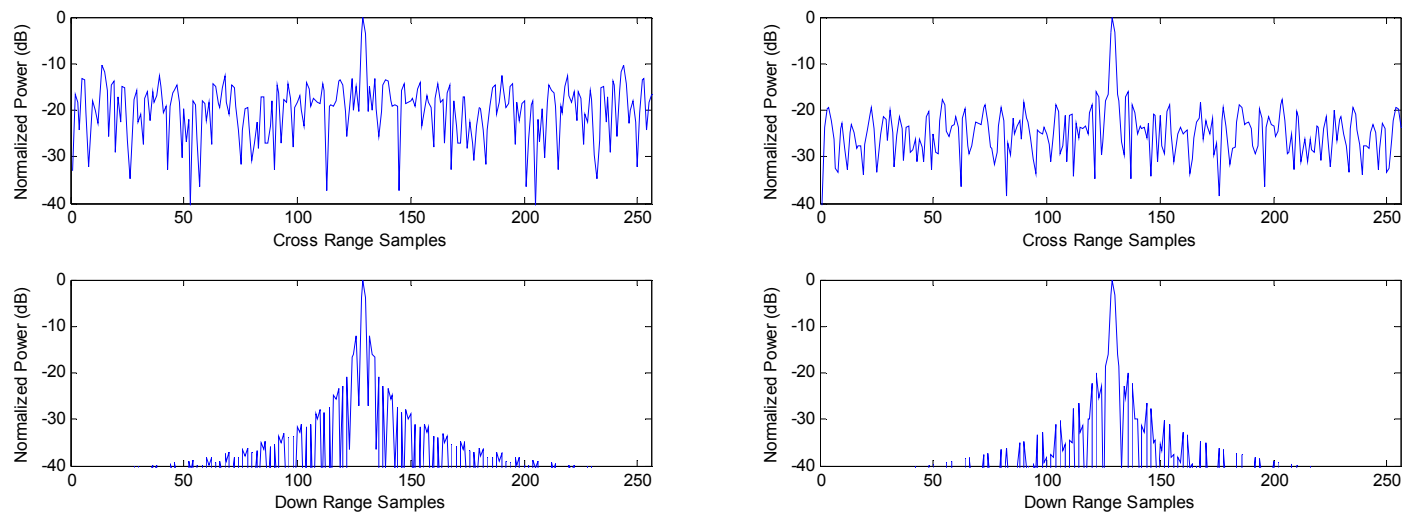

Figure 6-26: Initial (left) and final (right) PSR for the middle cell of the ROI. Population Size = 100, Archive Size $=10$. 


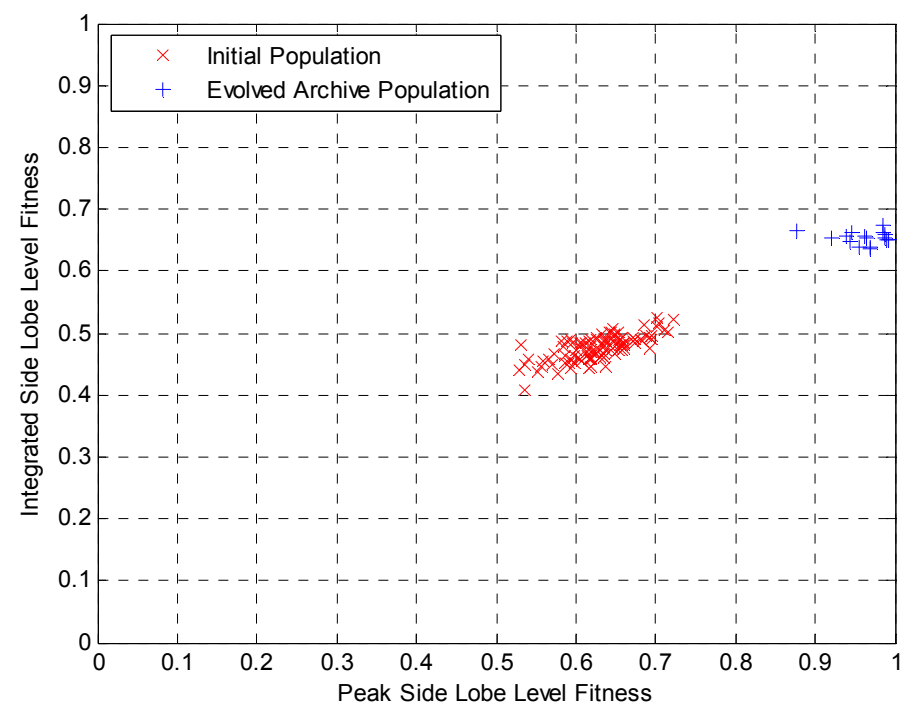

Figure 6-27: Initial population and final archive population for the split bandwidth, multiple subaperture experiment. Population Size $=100$, Archive Size $=20$.
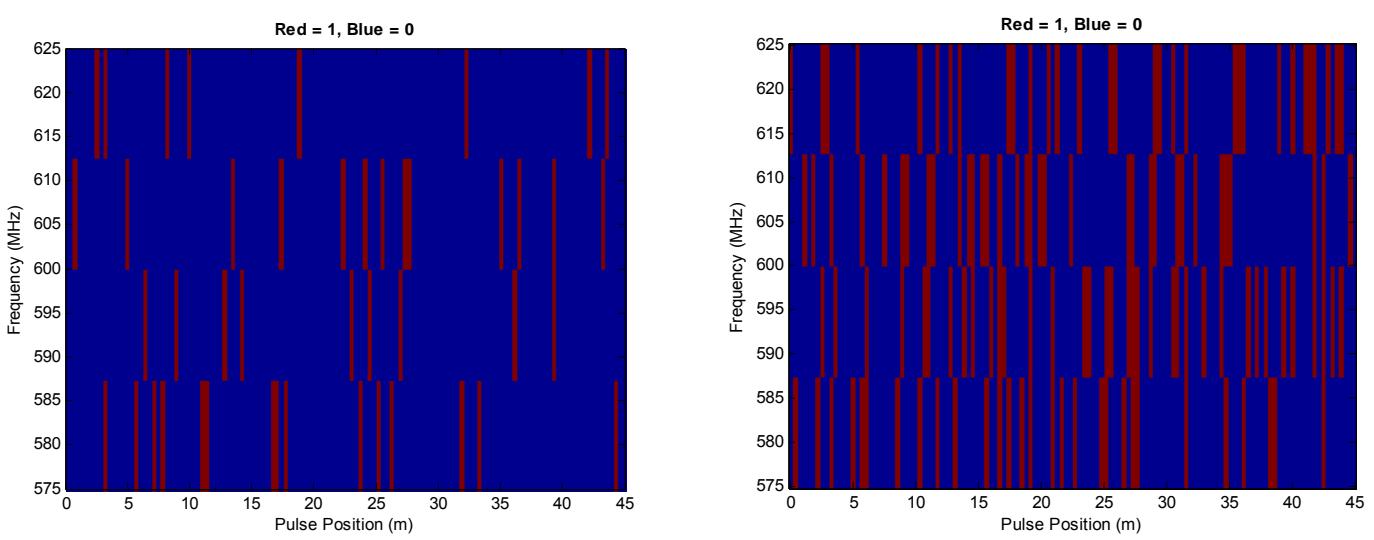

Figure 6-28: Initial (left) and final (right) VPH for the middle cell of the ROI. Population Size $=100$, Archive Size $=20$.
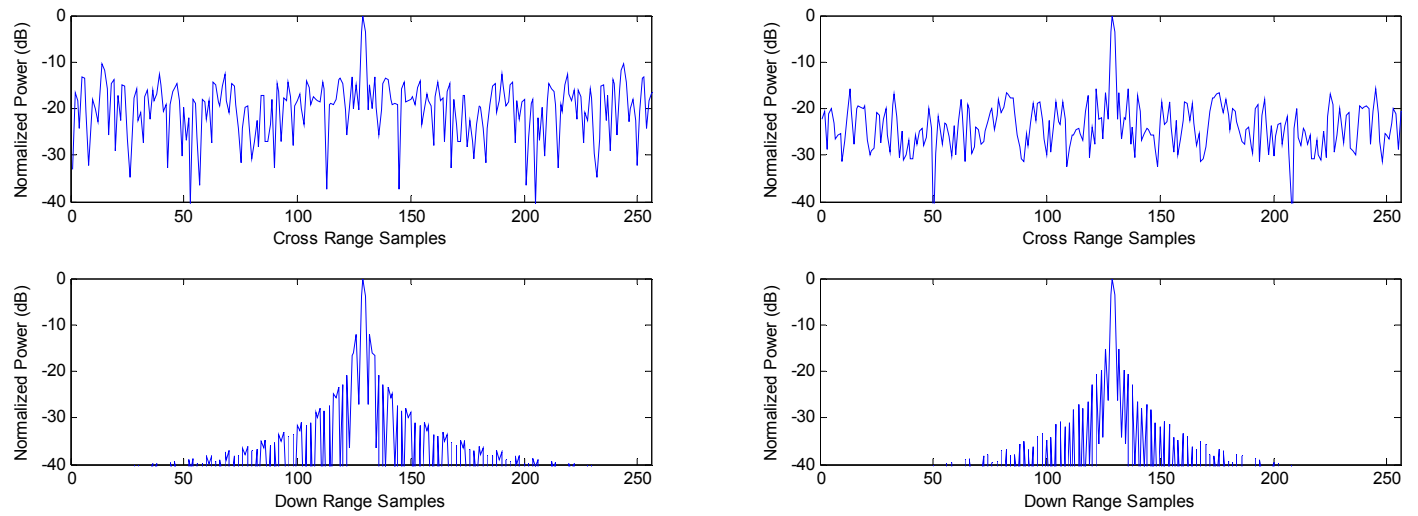

Figure 6-29: Initial (left) and final (right) PSR for the middle cell of the ROI. Population Size $=100$, Archive Size $=20$. 


\begin{tabular}{|c|c|c|c|c|c|c|c|c|c|}
\hline \multicolumn{5}{|c|}{50 Pop. Members - 10 Arch. Size - Middle Cell of ROI } & \multicolumn{5}{|c|}{100 Pop. Members - 10 Arch. Size - Middle Cell of ROI } \\
\hline Generation & PSL (dB) & PSL Fitness & ISL (dB) & ISL Fitness & Generation & PSL (dB) & PSL Fitness & ISL (dB) & ISL Fitness \\
\hline 0 (initial) & -7.48 & 0.53 & -32.13 & 0.48 & 0 (initial) & -7.48 & 0.53 & \begin{tabular}{|l|}
-32.13 \\
\end{tabular} & 0.48 \\
\hline 50 & -14.43 & 1.00 & -36.11 & 0.64 & 50 & -14.37 & 1.00 & -36.08 & 0.64 \\
\hline 100 & -14.62 & 1.00 & -36.73 & 0.67 & 100 & -14.13 & 1.00 & -36.63 & 0.67 \\
\hline 200 & -13.77 & 0.98 & -36.11 & 0.64 & 200 & -14.36 & 1.00 & -36.95 & 0.68 \\
\hline 300 & -13.77 & 0.98 & -36.11 & 0.64 & 300 & -14.72 & 1.00 & -36.68 & 0.67 \\
\hline 400 & -13.77 & 0.98 & -36.11 & 0.64 & 400 & -15.49 & 1.00 & -37.60 & 0.70 \\
\hline 500 & -13.77 & 0.98 & -36.11 & 0.64 & 500 & -14.64 & 1.00 & -37.95 & 0.72 \\
\hline \multicolumn{5}{|c|}{50 Pop. Members - 20 Arch. Size - Middle Cell of ROI } & \multicolumn{5}{|c|}{100 Pop. Members - 20 Arch. Size - Middle Cell of ROI } \\
\hline Generation & PSL (dB) & PSL Fitness & ISL (dB) & ISL Fitness & Generation & PSL (dB) & PSL Fitness & ISL $(\mathrm{dB})$ & ISL Fitness \\
\hline 0 (initial) & -7.48 & 0.53 & -32.13 & 0.48 & 0 (initial) & -7.48 & 0.53 & -32.13 & 0.48 \\
\hline 50 & -13.46 & 0.96 & -35.95 & 0.64 & 50 & -13.60 & 0.97 & -36.33 & 0.65 \\
\hline 100 & -14.35 & 1.00 & -36.02 & 0.64 & 100 & -15.18 & 1.00 & -36.48 & 0.66 \\
\hline 200 & -14.58 & 1.00 & -36.67 & 0.67 & 200 & -14.49 & 1.00 & -36.96 & 0.68 \\
\hline 300 & -14.05 & 1.00 & -36.39 & 0.66 & 300 & -14.81 & 1.00 & -36.85 & 0.67 \\
\hline 400 & -14.64 & 1.00 & -36.46 & 0.66 & 400 & -15.74 & 1.00 & -37.59 & 0.70 \\
\hline 500 & -14.69 & 1.00 & -36.84 & 0.67 & 500 & -15.74 & 1.00 & -37.59 & 0.70 \\
\hline
\end{tabular}

Table 6-4: Summary of the evolution of PSL and ISL values for each split bandwidth, multiple subaperture simulation

The 100 population, 10 archive size experiment was repeated using five different initial random seeds. This was done to verify the results that were obtained. The following fitness plot depicts the initial population and the final archive population for the five starting seeds, each represented by a different color. Figure 6-31 shows a closer view of the archive population of Figure 6-30.

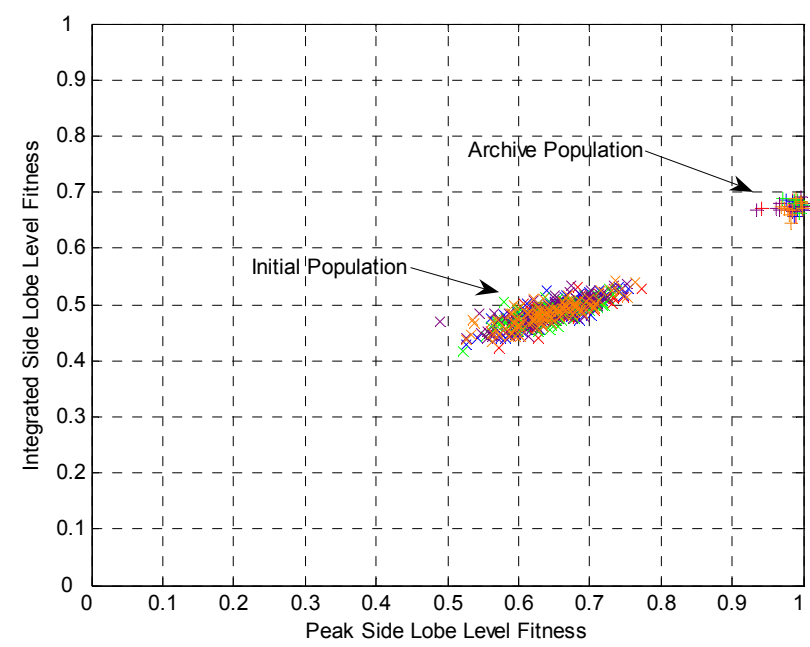

Figure 6-30: Initial population and final archive population using five different random seeds for the split bandwidth, multiple sub-aperture experiment. Population Size $=100$, Archive Size $=10$. 


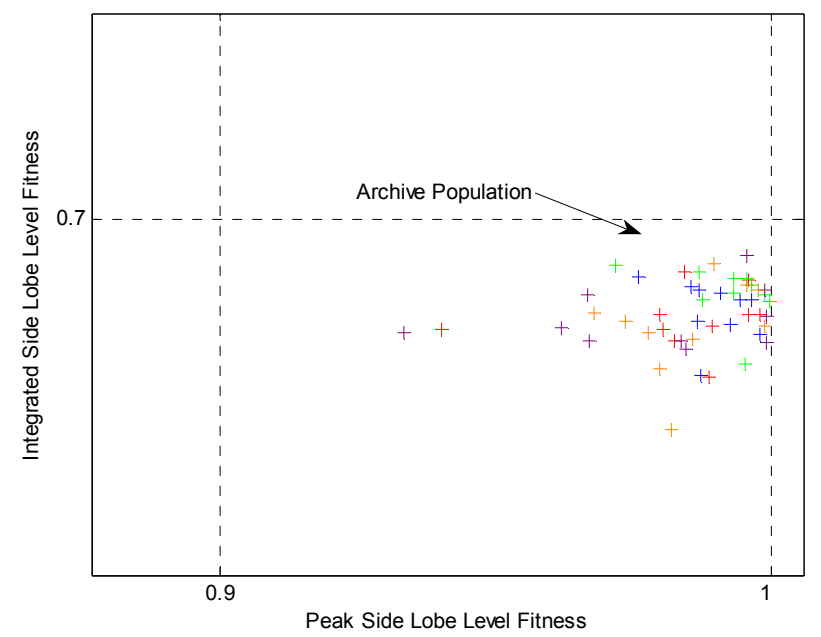

Figure 6-31: Archive population using five different random seeds for the split bandwidth, multiple sub-aperture experiment. Population Size $=100$, Archive Size $=10$.

\subsubsection{Analysis}

Like the previous experiment, the final archive population showed an improvement in fitness over the initial random population. However, compared to the previous experiment it was shown that the PSL fitness was higher and the ISL fitness was lower for this experiment on average. By splitting the VPH into four regions in the downrange dimension, some structure was introduced in that dimension of the PSR. This is a direct cause for the decreased ISL fitness. The increase in PSL fitness could have been an effect of this spreading of the PSR energy as well. The peak level of the normalized power may decrease but the integrated power increases as it spreads out over the 2-D region. Also like the previous experiment, the best results in terms of PSL and ISL fitness seemed to be a product of the simulation that used 100 population members and 10 archive members. This is not surprising as more population members allows SPEA2 to have more genetic data to choose from, and a smaller archive ensures that only the best 
solutions reproduce. Also as in the previous experiment, there are empty regions within the VPH of the best evolved archive member. There are pulse positions and frequency spectrum that is available to accomplish other missions.

\subsection{Scaled Scenario 2: MTI Mission}

The second scaled simulation scenario was generated to accomplish an MTI mission using number of integrated pulses and revisit time as objectives. Section 5.3.2 and 5.3.3 discuss the importance of these objectives in relation to an MTI mission. The scaled physical scenario is depicted in Figure 6-32.

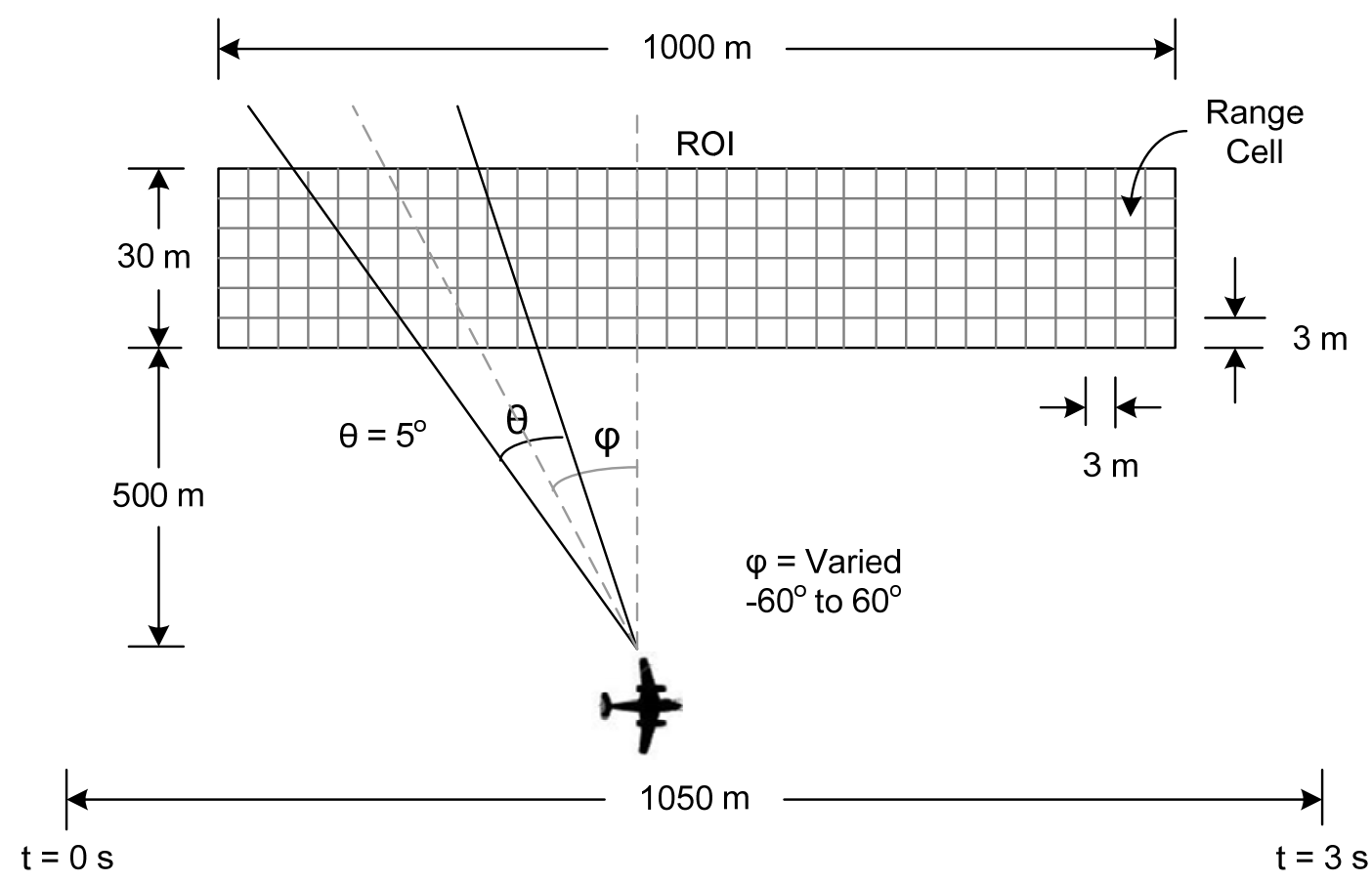

Figure 6-32: Scenario 2 - MTI mission (not drawn to scale)

While obviously not drawn to scale, Figure 6-32 provides an effective visual representation of the simulated scenario. As many of the scenario parameters as possible were kept the same as SAR mission scenario for consistency. In order to make it possible 
to achieve a revisit time specification, the scenario had to be set up to run for a longer mission time. Three seconds of simulation time with a revisit time goal of $0.75 \mathrm{~s}$ was selected for this scenario. Attempting four revisits of each cell was sufficient to determine the effectiveness of a scaled mission. A longer simulation time increased the cross-range dimension of the VPH by introducing many more pulse positions. At a medium PRF of $333 \mathrm{~Hz}, 1000$ pulses are generated in $3 \mathrm{~s}$. Thus, an IFFT size of at least 256 x 1000 would be required to achieve a resolution accurate enough to even coarsely determine a PSL and ISL value. As discussed at the beginning of this chapter, performing large IFFT operations on many range cells is not possible given the computational constraints of a single PC. With an IFFT operation no longer considered and since revisit time and number of integrated pulses is easy to calculate for a large number of cells, it was possible to increase the size of the ROI to conform to the increase in mission time. The dimensions and placement of the ROI as well as the varying azimuth pointing angle guarantee that it was physically possible to illuminate each cell at all times during the mission.

\subsubsection{Revisit Time \& Integrated Pulses Experiment}

The experiment performed with the MTI scaled scenario used a single aperture with a center frequency of $600 \mathrm{MHz}$. The azimuth pointing angle was allowed to be varied between -60 to 60 degrees in 3.75 degree increments, requiring five bits of encoding per CPI. At each CPI, the radar was permitted to emit 1, 8, 16 or 32 pulses. Each pulse that was emitted during a CPI had the same azimuth pointing angle. Two bits were used to encode the number of pulses. Depending on the choice for the number of pulses per CPI, 
the waveform suite varies in length. A visual representation of a waveform suite for this experiment can be seen in Figure 6-33.

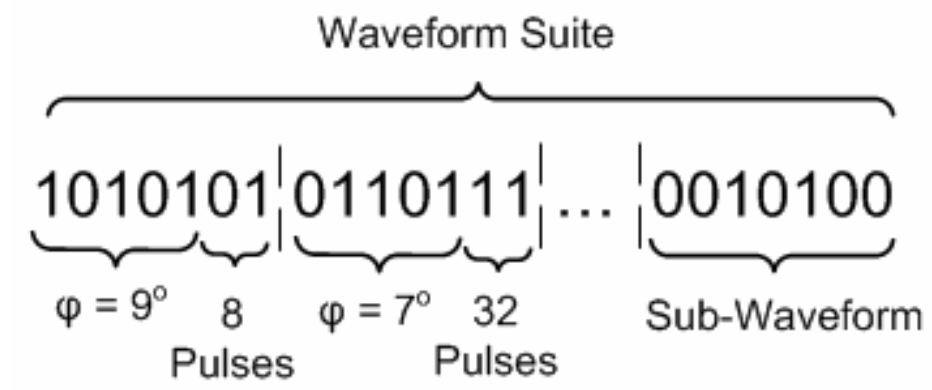

Figure 6-33: Example waveform suite for the revisit time \& integrated pulses experiment

As the radar illuminated a range cell, a timestamp and the number of pulses placed on the cell was recorded. The difference in timestamps was then calculated to determine revisit times for each cell. The objective functions shown in Figure 5-5 and Figure 5-6 were then applied to each cell to determine a pulse fitness and revisit time fitness value for each instance of a revisit time and each number of pulses that were placed on that cell. These fitness values were then averaged first within their cell and then over the entire ROI to determine a single number of pulse fitness for a waveform suite, or individual. An example of this process for one single range cell is shown in Table 6-5.

\begin{tabular}{|c|c|c|}
\hline Revisit Times (s) & Revisit Time Fitness Values & Avg. Cell Revisit Time Fitness \\
\hline $0.50,0.65,1.10$ & $0.67,0.87,0.53$ & 0.69 \\
\hline Number of Pulses & Pulse Fitness Values & Avg. Cell Pulse Fitness \\
\hline $8,32,16$ & $0.25,1.00,0.50$ & 0.58 \\
\hline
\end{tabular}

Table 6-5: Single range cell fitness evaluation for revisit time $\&$ integrated pulses experiment

The revisit time \& integrated pulses experiment was performed using different population and archive sizes in SPEA2. Four different simulations were run that alternated population sizes of $50 \& 100$ members and archive sizes of $10 \& 20$. Also, to 
demonstrate the increased effectiveness of using a larger population size, a simulation was also run with 200 population members and an archive size of 25 members. This simulation was run over several days and reiterates the need for a multiple PC cluster to run large populations and more complicated scenarios. A summary of the genetic and radar parameters used in this experiment are presented in Table 6-6.

\begin{tabular}{|l|c|}
\hline Simulation Parameter & Value \\
\hline No. of sub-apertures & 1 \\
\hline Physical apterture size & $6 \mathrm{~m}$ \\
\hline Flight distance & $1050 \mathrm{~m}$ \\
\hline Center frequency & $600 \mathrm{MHz}$ \\
\hline Bandwidth & $50 \mathrm{MHz}$ \\
\hline PRF & $333 \mathrm{~Hz}$ \\
\hline No. of pulses (varied) & $1,8,16,32$ \\
\hline Cross range cell size & $3 \mathrm{~m}$ \\
\hline Down range cell size & $3 \mathrm{~m}$ \\
\hline Beamwidth & $5^{\circ}$ \\
\hline Airplane velocity & $130 \mathrm{~m} / \mathrm{s}$ \\
\hline ROI cross range size & $1000 \mathrm{~m}$ \\
\hline ROI down range size & $30 \mathrm{~m}$ \\
\hline Distance to ROI & $500 \mathrm{~m}$ \\
\hline Azimuth angle (varied) & $-60^{\circ}$ to $60^{\circ}$ in 3.75 steps \\
\hline Objective functions & Revisit time $\&$ No. of pulses \\
\hline Simulation time & $3 \mathrm{~s}$ \\
\hline Crossover probability & 0.5 \\
\hline Mutation probability & 0.05 \\
\hline SPEA2 archive size & 10 or $20 \mathrm{members}$ \\
\hline SPEA2 population size & 50 or $100 \mathrm{members}$ \\
\hline Generations & 500 \\
\hline
\end{tabular}

Table 6-6: Summary of simulation parameters for the revisit time \& integrated pulses experiment

\subsubsection{Results}

The following figures display the results from simulations run with all combinations of population and archive size. The fitness values of the initial and final population members are presented. 


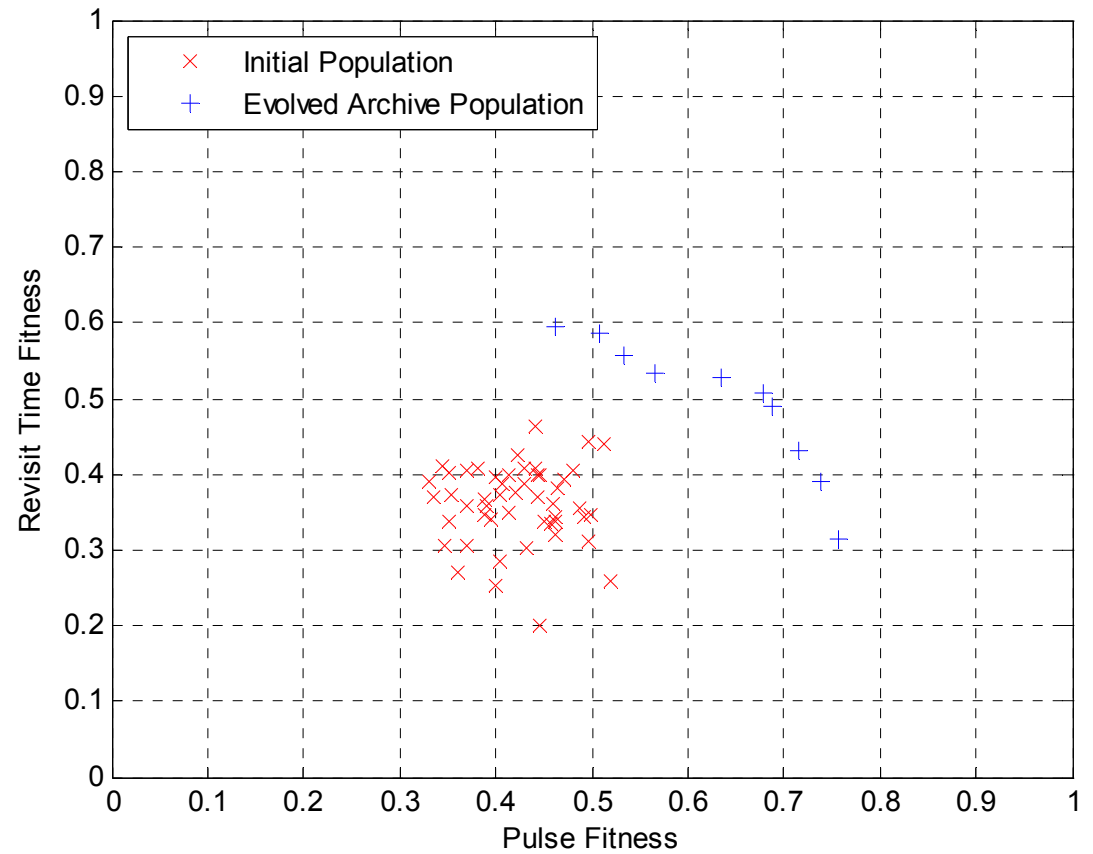

Figure 6-34: Initial population and final archive population for the revisit time \& integrated pulses experiment. Population Size $=$ 50, Archive Size $=10$.

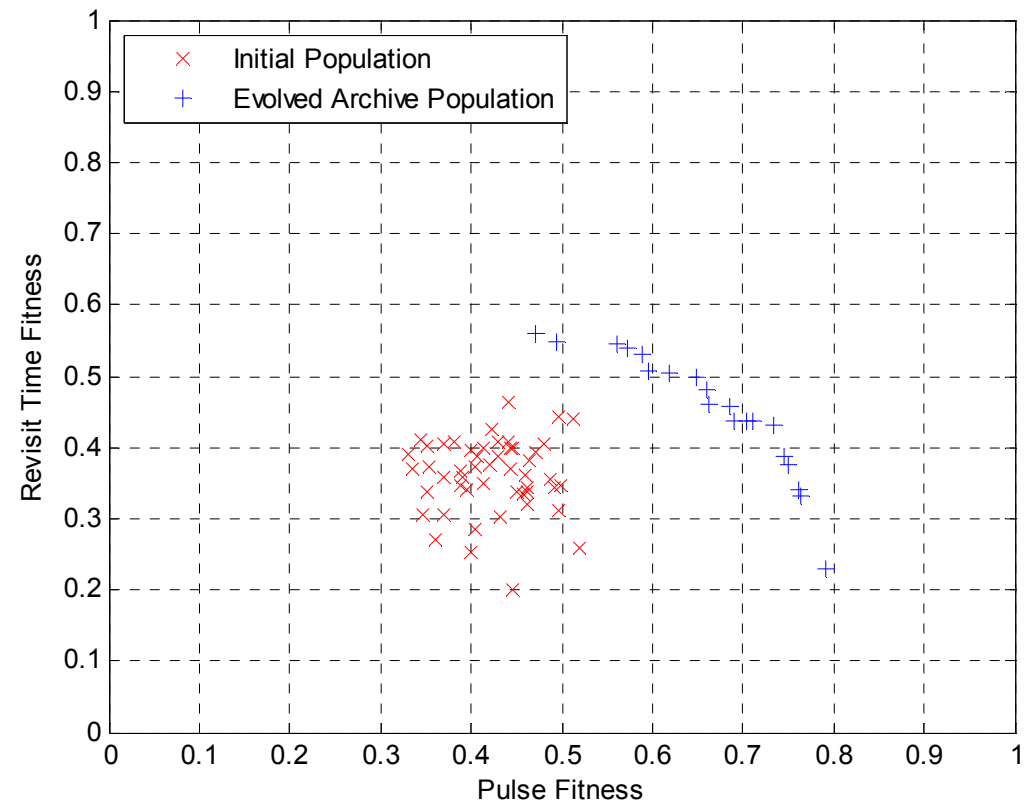

Figure 6-35: Initial population and final archive population for the revisit time \& integrated pulses experiment. Population Size $=$ 50, Archive Size $=20$. 


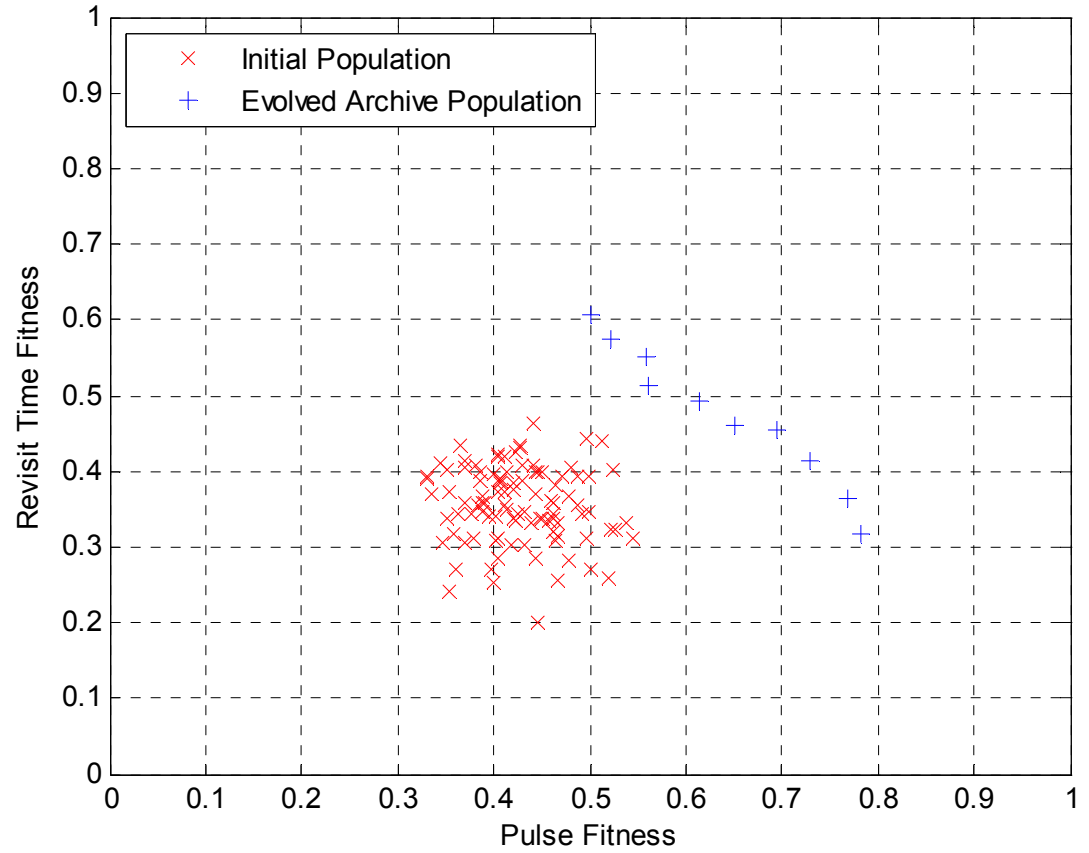

Figure 6-36: Initial population and final archive population for the revisit time \& integrated pulses experiment. Population Size $=100$, Archive Size $=10$.

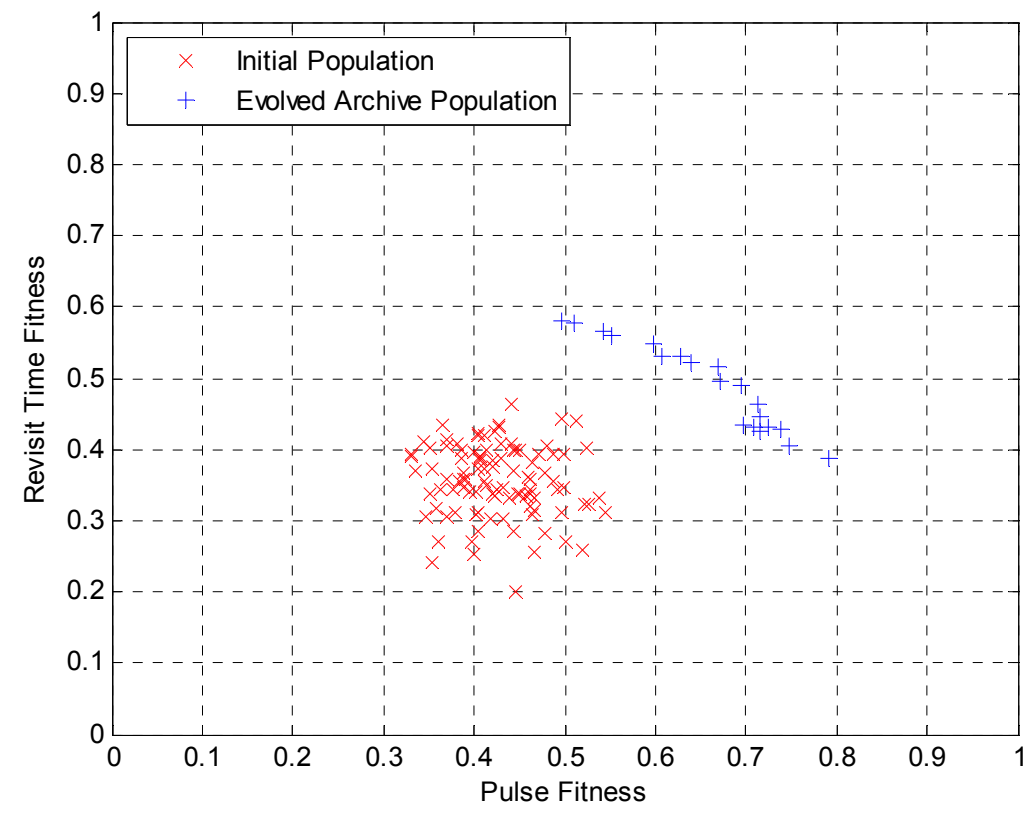

Figure 6-37: Initial population and final archive population for the revisit time \& integrated pulses experiment. Population Size $=100$, Archive Size $=20$. 


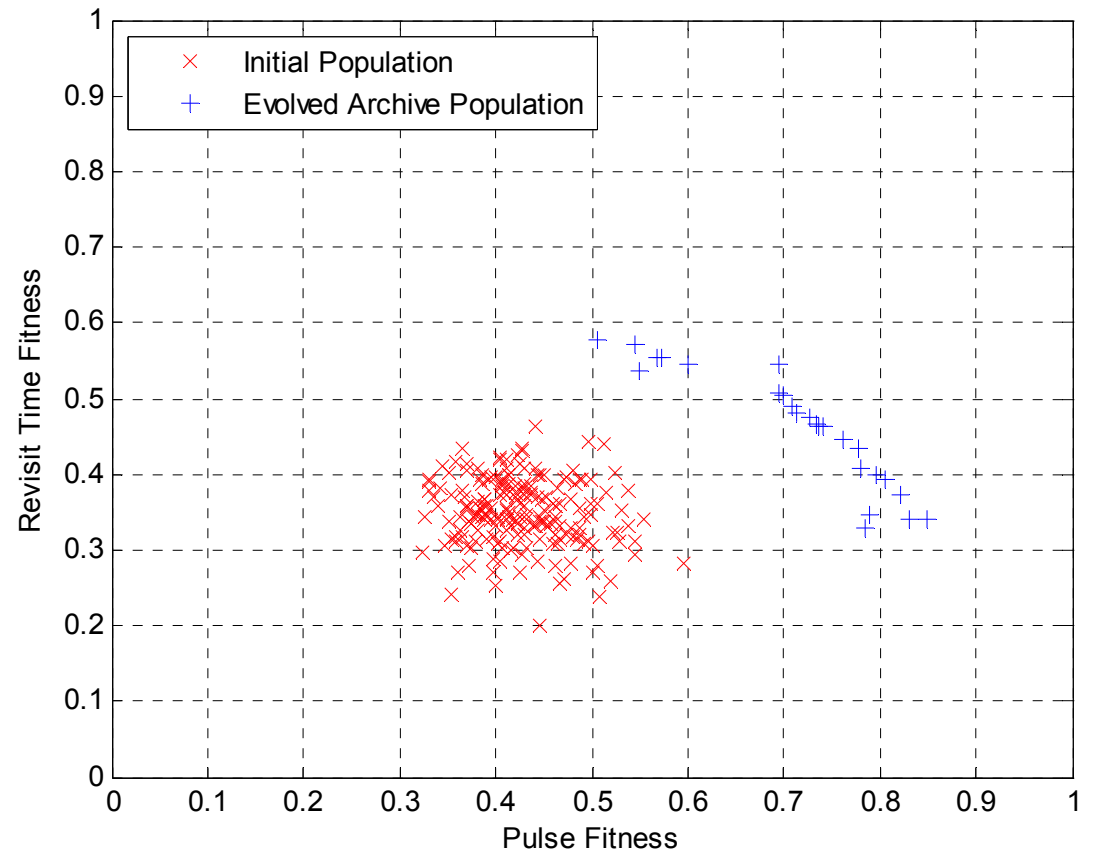

Figure 6-38: Initial population and final archive population for the revisit time \& integrated pulses experiment. Population Size $=200$, Archive Size $=25$.

The 100 population, 10 archive size experiment was repeated using five different initial random seeds. This was done to verify the results that were obtained. The following fitness plot depicts the initial population and the final archive population for the five starting seeds, each represented by a different color. 


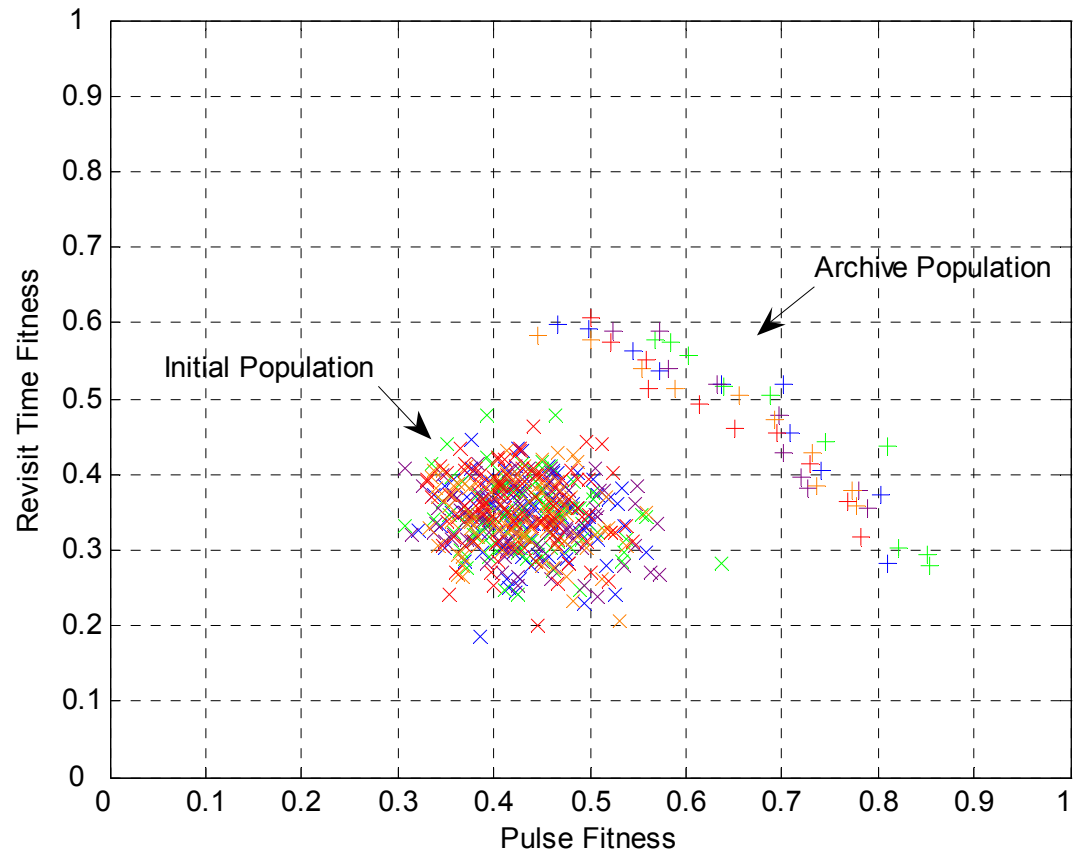

Figure 6-39: Initial population and final archive population using five different random seeds for the revisit time $\&$ integrated pulses experiment. Population Size $=100$, Archive Size $=10$.

\subsubsection{Analysis}

Each simulation run generated a well-spread Pareto front for the revisit time \& integrated pulses experiment. Unlike PSL and ISL which are related, revisit time and number of integrated pulses are conflicting objectives. The integrated pulses objective is attempting to look at each cell as much as possible and place as many pulses on the cell as it can. The revisit time objective tries to establish an order in which the cells are to be illuminated by the radar. When multiple pulses are used, they each have the same azimuth pointing angle. Keeping this angle constant for many pulses fundamentally opposes the revisit time objective. Therefore, the results show a diversified Pareto front in which there are some solutions with high pulse fitness and low revisit time fitness and vice-versa. 
Obviously, the goal of these experiments is to increase each fitness value to one and move the Pareto front toward the upper right of the fitness plot. The results presented here indicate that the front is indeed moving in this direction. As shown by Figure 6-38, a larger population size yields better results over the same number of generations. The best solution in this simulation that was run with 200 population members has a revisit time fitness of approximately 0.55 and a pulse fitness of about 0.7 whereas the simulations run with smaller population sizes achieve a best solution of approximately 0.5 revisit time fitness and 0.65 pulse fitness. This may not seem like a substantial difference, but if the algorithm was allowed to run with thousands of population members thousands of generations, SPEA2 would have more of a chance to search the solution space and produce highly fit solutions. 


\section{Conclusion and Future Work}

\subsection{Conclusion}

Chapter 6 presented results that demonstrate the effectiveness of SPEA2 for designing waveforms to accomplish missions with multiple objectives. Each simulation for every scaled experiment showed an increase in fitness for all objectives from the initial population to the final archive population. Regardless of what the actual fitness values were, each experiment produced a significant shift toward Pareto optimal solutions with higher fitness values, corresponding to increased mission effectiveness. It was demonstrated that SPEA2 can find Pareto solutions for related objectives as well as conflicting ones. It was also shown through the empty space in the final video phase histories (Figure 6-10, Figure 6-25) that there is available space within the waveform suite for a SAR mission to accomplish other missions, such as MTI. The results presented here for individual multi-objective scaled missions provide optimism for accomplishing a true multi-mission scenario experiment given more computational resources. This evolutionary technique is especially encouraging because it has shown the ability design waveforms that can effectively accomplish multiple user defined radar mission objectives.

\subsection{Future Work}

Waveform design is a task that can be done a-priori. In the case of this work, the goal was to apply a genetic algorithm to design a radar waveform that can accomplish 
multiple missions that have multiple objectives. Since the design is a-priori, the amount of time that the genetic algorithm requires to find a solution, within reason, is inconsequential. Therefore, even if the algorithm required days, weeks, or months to run a sophisticated multi-mission scenario, the resulting waveform can be used in real-world applications.

With this in mind, the next step in this work is to integrate the SAR mission scenario with the MTI mission scenario and use all four objectives for a single design. As noted earlier, this integration can not be accomplished using a single PC due to the number and large size of the required 2-D IFFT operation for the SAR PSL and ISL objective functions. However, implementing this integrated scenario using a parallel processing system with multiple nodes can be accomplished and is the next major task. Essentially, the MTI scenario described in Section 6.2 can be used for the experiment by calculating a VPH for each cell as done in the SAR experiments and adding the PSL and ISL fitness values into the revisit time and number of pulses fitness vector. SPEA2 would then operate upon a 4 x 1 fitness vector rather than a 2 x 1 fitness vector as done in each of the experiments performed for this thesis.

Another improvement to the work done here that can be performed using a multinode cluster is to increase the number of SPEA2 population members and number of generations. As discussed at the end of Chapter 6, increasing these genetic parameters increases the size and, inherently the variety, of the possible solution space. A five percent increase in fitness values was demonstrated for the MTI mission when the population size was increase from 100 members to 200 members. Population sizes of 
around 100 members are considered small for a genetic algorithm. Typically, populations consisting of thousands of members and running for thousands of generations are implemented. This capacity would be possible with the use of a multi-node cluster and should be integrated into the future experiments.

Finally, other genetic operators such as alternate forms of selection, crossover and mutation could be implemented to possibly produce different Pareto solutions. Different genetic operators would most likely create minor differences in relation to the current results and were not explored in this thesis because the focus was to prove that SPEA2 could be applied to multi-objective radar waveform design. This goal has been accomplished and an experiment has been outlined to apply SPEA2 to a true multimission, multi-objective radar scenario using a parallel processing computer cluster. 


\section{References}

[1] V.J. Amuso and J. Enslin, The Strength Pareto Evolutionary Algorithm 2 Applied to Simultaneous Multi-mission Waveform Design, Third International Conference on Waveform Diversity and Design, June 2007, Pisa, Italy.

[2] E. Zitzler, M. Laumanns, and L. Thiele, "SPEA2: Improving the Strength Pareto Evolutionary Algorithm for Multiobjective Optimization,” Evolutionary Methods for Design, Optimization and Control with Application to Industrial Problems (EUROGEN 2001), K.C. Giannakoglou et al. (Ed), International Center for Numerical Methods in Engineering, 2002, pp. 95-100.

[3] M.L. Skolnik, Introduction to Radar Systems, McGraw-Hill, St. Louis, 1980.

[4] M.E. Tobin and M. Greenspan, "Smuggling Interdiction Using an Adaptation of the AN/APG-76 Multimode Radar," IEEE Aerospace and Electronics Systems Magazine, Vol. 11, Issue 11, November 1996, pp. 19-24.

[5] E. Zitzler and L. Thiele, "Multi-objective Evolutionary Algorithms: A Comparative Case Study and the Strength Pareto Approach,” IEEE Transactions on Evolutionary Computation, Vol. 3, No. 4, 1999, pp. 257-271.

[6] D. Collier and L. Goldstone, The Multi-mode Array-Improved Versatility through Enhanced SAR, IEEE Aerospace and Electronics Conference Proceedings, Vol. 1, May 1994, pp. 151-155.

[7] G.E. Haslam, M.R. Vant, D. DiFilippo, A High Resolution Synthetic Aperture Radar, IEEE International Radar Conference Proceedings, October 1992, pp. 371374.

[8] A. Damini, M. McDonald, and G.E. Haslam, X-band Wideband Experimental Airborne Radar for SAR, GMTI and Martime Surveillance, IEEE Radar, Sonar and Navigation Proceedings, Vol. 150, No. 4, August 2003, pp. 305-312.

[9] R. Strong, "Radar: The Evolution since World War II," IEEE Aerospace and Electronics Systems Magazine, Vol. 20, Issue 1, January 2005, pp. 19-24.

[10] M.E. Davis, B. Himed, and D. Zasada, Design of Large Space Based Radar for Multimode Surveillance, IEEE International Radar Conference Proceedings, May 2003, pp. 1-6. 
[11] H. Mendelson and W. Ogle, A Waveform Design Technique for Range and Doppler Ambiguity Mitigation, Second International Conference on Waveform Diversity and Design, January 2006, Honolulu, HI.

[12] P. Antonik, M. Wicks, H. Griffiths and C. Baker, Multi-Mission Multi-Mode Waveform Diversity, IEEE International Radar Conference Proceedings, April 2006, pp. 580-582.

[13] V.J. Amuso, P. Antonik, R. Schneible, Y. Zhang, Evolutionary Computation Approach to Multi Mission Waveform Design, RADAR 2002, Edinburgh, UK, October 2002, pp. 454-458.

[14] V.J. Amuso, P. Antonik, R. Schneible, Y. Zhang, A Strength Pareto Evolutionary Algorithm (SPEA) for Multi-Mission Radar Waveform Optimization, First International Conference on Waveform Diversity and Design, November 2004, Edinburgh, UK.

[15] M.L. Skolnik (Ed.), Radar Handbook, McGraw Hill, Boston, 1990.

[16] M. Soumekh, Synthetic Aperture Radar Signal Processing, John Wiley \& Sons, New York, 1999.

[17] C.A. Coello Coello, D.A. Van Veldihuizen, and G.B. Lamont, Evolutionary Algorithms for Solving Multi-Objective Problems, Kluwer Academic Publishers, New York 2002.

[18] J.M. Johnson and Y. Rahman-Samii, "Genetic Algorithms in Engineering Electromagnetics," IEEE Antennas and Propagation Magazine, Vol. 39, No. 4, August 1997, pp. 7-21.

[19] D.S. Weile and E. Michielssen, "Genetic Algorithm Optimization Applied to Electromagnetics: A Review," IEEE Transactions on Antennas and Propagation, Vol. 45, No. 3, March 1997, pp. 343-353.

[20] S.K. Kassicieh, T.L. Paez, and G. Vora, Investment Decisions using Genetic Algorithms, Proceedings of the Thirtieth International Conference on System Sciences, January 1997, Vol. 5, pp. 484-490.

[21] V. Cristea and G. Godza, Genetic Algorithms and Intrinsic Parallel Characteristics, Proceedings of the 2000 Congress on Evolutionary Computation, July 2000, Vol. 1, pp. 431-436.

[22] G. Rudolph, "Convergence Analysis of Canonical Genetic Algorithms," IEEE Transactions on Neural Networks, Vol. 5, No. 1, January 1994, pp. 96-101. 
[23] B. Zhang and J. Kim, "Comparison of Selection Methods for Evolutionary Optimization”, Evolutionary Optimization: An International Journal on the Internet, Vol. 2, No. 1, 2000, pp. 55-70.

[24] R. Tinos and A.C.P.L.F. de Carvalho, A Genetic Algorithm with Gene Dependent Mutation Probability for Non-Stationary Optimization Problems, Proceedings of the 2004 Congress on Evolutionary Computation, June 2004, Vol. 2, pp. 12781285 .

[25] A. Osyczka, Design Optimization, Academic Press, 1985, Chapter 7, pp. 193-227.

[26] J.D. Schaffer, Multiple Objective Optimization with Vector Evaluated Genetic Algorithms, Genetic Algorithms and their Applications: Proceedings of the First International Conference on Genetic Algorithms, pp. 93-100, 1985.

[27] D.E. Goldberg, Genetic Algorithms in Search, Optimization and Machine Learning, Addison-Wesley Publishing, Reading, Massachusetts 1989.

[28] C.A. Coello Coello, "Evolutionary Multi-objective Optimization: A Historical View of the Field”, IEEE Computational Intelligence Magazine, Vol. 1, Issue 1, February 2006, pp. 28-36.

[29] E. Zitzler, L. Thiele, and K. Deb, "Comparison of Multiobjective Evolutionary Algorithms: Empircal Results," IEEE Transactions on Evolutionary Computation, Vol. 8, No. 2, 2000 pp. 173-195.

[30] J.D. Knowles and D.W. Corne, "Approximating the Nondominated using the Pareto Archived Evolution Strategy," IEEE Transactions on Evolutionary Computation, Vol. 8, No. 2, 2000 pp. 149-172.

[31] K. Deb, A. Pratap, S. Agarwal, and T. Meyarivan, "A Fast and Elitist Multiobjective Genetic Algorithm: NSGA-II," IEEE Transactions on Evolutionary Computation, Vol. 6, No. 2, April 2002, pp. 182-197.

[32] N. Srinivas and K. Deb, "Multiobjective Optimization Using Nondominated Sorting in Genetic Algorithms," IEEE Transactions on Evolutionary Computation, Vol. 2, No. 3, Fall 1994, pp. 221-248.

[33] B.W. Silverman, "Density Estimation for Statistics and Data Analysis," Monographs on Statistics and Applied Probability, Chapman and Hall, London, 1986. 
[34] H. Kellerer, U. Pferschy, and D. Pisinger, Knapsack Problems, Springer, London, 2004.

[35] M. Jeong, "A Fourier Transform Based Sidelobe Reduction Method in Ultrasound Imaging," IEEE Transactions on Ultrasonics, Ferroelectrics, and Frequency Control, Vol. 47, No. 3, May 2000, pp. 759-763.

[36] R. Bose, A. Freedman, and B.D. Steinberg, "Sequence CLEAN: A Modified Deconvolution Technique for Microwave Images of Contiguous Targets," IEEE Transactions on Aerospace and Electronic Systems, Vol. 38, No. 1, January 2002, pp. 89-97.

[37] S.R. DeGraaf, "Sidelobe Reduction via Adaptive FIR Filtering in SAR Imagery," IEEE Transactions on Image Processing, Vol. 3, No. 3, May 1994, pp. 292-301.

[38] S. Holm, P.A. Valand, and K. Eldhuset, Performance of Cesar ERS-1 SAR Processor, Geoscience and Remote Sensing Symposium, Vol. 1, June 3-6, 1991, pp. 291-294.

[39] B.H. Smith, "An Analytic Nonlinear Approach to Sidelobe Reduction,” IEEE Transactions on Image Processing, Vol. 10, No. 8, August 2001, pp. 1162-1168. 